Subvariedades de ângulo constante em 3variedades homogêneas

\author{
Aline de Moraes Teixeira
}





\title{
Subvariedades de ângulo constante em 3-variedades homogêneas
}

\author{
Aline de Moraes Teixeira \\ Orientadora: Profa. Dra. Irene Ignazia Onnis \\ Dissertação apresentada ao Instituto de Ciências \\ Matemáticas e de Computação - ICMC-USP, como \\ parte dos requisitos para obtenção do título de Mestre \\ em Ciências - Matemática. VERSÃO REVISADA
}

USP - São Carlos

Maio de 2015 
Ficha catalográfica elaborada pela Biblioteca Prof. Achille Bassi e Seção Técnica de Informática, ICMC/USP, com os dados fornecidos pelo(a) autor(a)

d262s $\begin{aligned} & \text { de Moraes Teixeira, Aline } \\ & \text { Subvariedades de ângulo constante em 3-variedades } \\ & \text { homogêneas / Aline de Moraes Teixeira; orientadora } \\ & \text { Irene Ignazia Onnis. -- São Carlos, 2015. } \\ & 93 \mathrm{p.} \\ & \text { Dissertação (Mestrado - Programa de Pós-Graduação } \\ & \text { em Matemática) -- Instituto de Ciências Matemáticas } \\ & \text { e de Computação, Universidade de São Paulo, 2015. } \\ & \text { 1. Geometria Riemanianna. 2. Subvariedades de } \\ & \text { ângulo constante. 3. Variedades homogêneas. I. } \\ & \text { Ignazia Onnis, Irene, orient. II. Título. }\end{aligned}$


A todos que acreditaram em mim, quando eu mesma não acreditei. 



\section{Agradecimentos}

Primeiramente, a Deus por ter me capacitado, dado coragem para realizar este trabalho e ter me confortado nos momentos difíceis.

Aos meus pais Antônio e Vitalina e ao meu irmão Marcelo que, apesar da distância, sempre se fizeram presentes com seu apoio, carinho e orações.

Aos meus amigos de classe Alex, Liliam, Maycon, Camila, Samanta, Roberto e Jean pela força, ajuda, companheirismo e discussões muito produtivas ao longo desses dois anos.

Às minhas amigas de coração Mônica, Jéssica e Joice pela sua amizade, carinho e paciência ao me consolarem nos momentos de desespero.

À minha orientadora, a professora Irene I. Onnis, por todos esses anos de orientação, desde os tempos de iniciação científica, pela sua paciência, apoio e principalmente, por acreditar em mim.

Também agradeço aqueles que, diretamente ou indiretamente, colaboraram e que por ventura eu possa não ter lembrado.

Por fim, agradeço à CAPES e ao CNPq pelo apoio financeiro. 

Um resultado clássico enunciado por M.A. Lancret em 1802 e provado por B. de Saint Venant em 1845 é: uma condição necessária e suficiente para que uma curva forme um ângulo constante com respeito a um campo de Killing unitário de $\mathbb{R}^{3}$ é que a razão entre a curvatura e a torção seja constante. Curvas deste tipo são chamadas hélices generalizadas. O problema de Lancret-de Saint Venant foi generalizado para curvas em outras variedades de dimensão três como, por exemplo, as formas espaciais e os grupos de Lie.

Outra maneira de generalizar o estudo anterior é passar de curvas para superfícies, ou seja estudar as superfícies orientadas de 3-variedades Riemannianas cuja normal unitária faz um ângulo constante com certos campos de vetores "privilegiados" do espaço ambiente.

Nesta dissertação estudaremos os resultados obtidos em [16, 24, 26, 27] sobre a classificação de curvas e superfícies de ângulo constante nas seguintes 3 -variedades homogêneas: $\mathbb{R}^{3}$, o grupo de Heisenberg tridimensional e as esferas de Berger. 

A classical result stated by M.A. Lancret in 1802 and first proved by B. de Saint Venant in 1845 is: a necessary and sufficient condition in order to a curve makes a constant angle with respect a unit Killing vector field of $\mathbb{R}^{3}$ is that the ratio of curvature to torsion be constant. Such curves are called general helix. The problem of Lancret-de Saint Venant has been generalized to curves in other three-dimensional manifolds as, for example, the space forms and the Lie groups.

Another way to generalize the previous study is to pass from curves to surfaces, i.e. to study the oriented surfaces of Riemannian 3-manifolds for which the unit normal makes a constant angle with "favored" vector fields of the ambient space.

In this dissertation we will study the results obtained in [16, 24, 26, 27] about the classification of constant angle curves and surfaces in the following homogeneous 3-manifolds: $\mathbb{R}^{3}$, the three-dimensional Heisenberg group and the Berger sphere. 

1 Superfícies de ângulo constante em $\mathbb{R}^{3} \quad 5$ 1.1 Preliminares . . . . . . . . . . . . . . . . . . . . 6

1.2 Caracterização das superfícies de ângulo constante . . . . . . . . . . . . . 9

2 Curvas e superfícies em $\mathbb{R}^{3}$ que fazem ângulo constante com campos de Killing 17 2.1 Alguns resultados sobre curvas em $\mathbb{R}^{3} \ldots \ldots \ldots \ldots \ldots$

2.2 Classificação das superfícies que fazem ângulo constante com $V \ldots$. . . . . . . 26

3 Superfícies de ângulo constante no grupo de Heisenberg 37

3.1 Espaços homogêneos de dimensão três . . . . . . . . . . . . . . . . . . 38

3.1.1 Os espaços de Bianchi-Cartan-Vranceanu . . . . . . . . . . . . . . . 39

3.1.2 Imersões isométricas em BCV-espaços . . . . . . . . . . . . . . . 44

3.1.3 Superfície de ângulo constante nos BCV-espaços . . . . . . . . . . . . 47

3.2 o grupo de Heisenberg $\ldots \ldots \ldots \ldots \ldots \ldots \ldots$ 
3.3 Preliminares . . . . . . . . . . . . . . . . . . . . . . . 54

3.4 Superfícies de ângulo constante em $\mathbb{H}_{3} \ldots \ldots \ldots \ldots \ldots$

4 Superfícies de ângulo constante nas esferas de Berger 59

4.1 As esferas de Berger . . . . . . . . . . . . . . . . . . 60

4.1 .1 Estrutura Riemanniana das esfera de Berger . . . . . . . . . . . 61

4.2 Superfícies de ângulo constante . . . . . . . . . . . . . . . . . . 62

4.2 .1 o resultado principal . . . . . . . . . . . . . . . 70

\begin{tabular}{|ll} 
A Imersões isométricas e campos de Killing & 81
\end{tabular}

A.1 Equações fundamentais de uma imersão isométrical . . . . . . . . . . . . . . 82

A.2 Campos de Killing . . . . . . . . . . . . . . . . . . . 85

$\begin{array}{lr}\text { Bibliografia } & 90\end{array}$ 
O principal objetivo desse trabalho é expor alguns resultados recentes e importantes sobre o estudo das superfícies de ângulo constante em variedades Riemannianas homogêneas de dimensão três. Lembramos que uma superfície orientada é dita de ângulo constante se a normal unitária forma um ângulo constante com um campo de direções do espaço ambiente e, também, que uma variedade Riemanniana é dita homogênea se o seu grupo de isometrias age transitivamente sobre ela.

Cartan classificou os espaços homogêneos tridimensionais simplesmente conexos dependendo da dimensão do grupo de isometrias, que pode ser 3,4 ou 6 . No caso da dimensão ser 6 , a variedade é uma forma espacial, ou seja, o espaço Euclidiano $\mathbb{R}^{3}$, a esfera tridimensional $\mathbb{S}^{3}$ ou o espaço hiperbólico tridimensional $\mathbb{H}^{3}$. Se a dimensão do grupo de isometrias for 4 , a variedade é isométrica aos espaços produtos $\mathbb{S}^{2} \times \mathbb{R}$ ou $\mathbb{H}^{2} \times \mathbb{R}$, ou a um dos seguintes grupos de Lie munidos de uma métrica invariante à esquerda: $\mathbb{H}_{3}$ (grupo de Heisenberg tridimensional), $\mathbb{S}_{\epsilon}^{3}$ (esferas de Berger), $\widetilde{S L}(2, \mathbb{R})$ (revestimento universal do grupo linear especial). No caso do grupo de isometrias ser de dimensão 3, a variedade possui a geometria do grupo de Lie $\mathrm{Sol}_{3}$.

Feita a exceção para $\mathbb{H}^{3}$, no caso da dimensão ser 4 ou 6 o espaço homogêneo é localmente isométrico a (uma parte de) $\mathbb{R}^{3}$, munido de uma métrica que depende de dois parâmetros reais. Tal família de métricas aparece primeiramente no trabalho [5] de L. Bianchi e, mais tarde, nos artigos [7. 34] de É. Cartan e G. Vranceanu, respectivamente. Por esta razão, estes espaços são conhecidos como variedades de Bianchi-Cartan-Vranceanu (ou BCV-espaços). 
Outro fato importante dos BCV-espaços que precisamos relembrar é que eles admitem uma submersão Riemanniana sobre uma superfície de curvatura Gaussiana constante, que é dita $f$ bração de Hopf pois generaliza a fibração de Hopf clássica $\pi: \mathbb{S}^{3}(k / 4) \longrightarrow \mathbb{S}^{2}(k)$ (ver a Definição 3.1.5).

Cronologicamente, o primeiro trabalho sobre superfícies de ângulo constante é [8], onde os autores provaram que se o espaço ambiente for o espaço Euclidiano 3-dimensional, estas superfícies possuem importantes aplicações na física, enquanto podem ser usadas para descrever interfaces que ocorrem em configurações de equilibrio de cristais líquidos. O primeiro capítulo deste trabalho é voltado ao estudo da classificação completa das superfícies de $\mathbb{R}^{3}$ que fazem ângulo constante com a direção $\mathbb{R}$ (ver Teorema 1.2.5), que foi apresentada em [26].

Também nos trabalhos [12] e [13] os autores consideram $\mathbb{R}$ como direção privilegiada do espaço ambiente e classificam as superfícies de ângulo constante nos espaços produto $\mathbb{S}^{2} \times \mathbb{R}$ e $\mathbb{H}^{2} \times \mathbb{R}$, respectivamente. Observe que nestes dois casos a fibração de Hopf é a projeção no primeiro fator $\pi: \mathbb{S}^{2} \times \mathbb{R} \rightarrow \mathbb{S}^{2}$ e $\pi: \mathbb{H}^{2} \times \mathbb{R} \rightarrow \mathbb{H}^{2}$ (respectivamente) e a direção $\mathbb{R}$ representa a direção das fibras.

No Capítulo2 estudaremos os resultados de [27]. Primeiramente serão consideradas as curvas planas e espaciais de $\mathbb{R}^{3}$ que fazem ângulo constante com o campo de Killing $V=-y \partial_{x}+x \partial_{y}$. Logo após, apresentaremos a classificação completa das superfícies orientadas cuja normal unitária faz ângulo constante com esse mesmo campo de Killing (ver Teorema 2.2.2), que inclui (entre outras) o catenóide e a superfície de Dini.

O terceiro capítulo é dedicado ao estudo dos resultados do artigo [16], no qual os autores estendem a noção de superfície de ângulo constante dada em [12, 13] para um espaço de BianchiCartan-Vranceanu geral da seguinte maneira: “uma superficie orientada num BCV-espaço é uma superfície de ângulo constante se o ângulo entre a normal unitária à superfície e e a direção tangente às fibras da fibração de Hopf é o mesmo em todos os pontos".

Ressaltamos que em [16] é apresentada a classificação local completa das superfícies de ângulo constante no grupo de Heisenberg e, também, algumas propriedades sobre as superfícies de ângulo constante nos restantes BCV-espaços que têm grupo de isometria de dimensão quatro. 
No Capítulo 4 apresentaremos os resultados do artigo [24] sobre o estudo das superfícies de ângulo constante na esfera de Berger, que é a esfera $\mathbb{S}^{3}$ munida com uma métrica de curvatura seccional positiva e não constante obtida "deformando" a métrica canônica de $\mathbb{S}^{3}$ (ver (4.1)). Neste contexto, o resultado principal que mostraremos é o Teorema 4.2.5 que afirma que uma superfície de ângulo constante na esfera de Berger é determinada por uma família a um parâmetro (apropriada) de isometrias do espaço ambiente e por uma geodésica de um 2-toro na esfera 3-dimensional.

A fim de auxiliar o leitor na compreensão deste trabalho, no Apêndice A recordaremos as equações básicas de uma imersão isométrica entre variedades Riemannianas e, também, a definição de campo de Killing e alguns resultados que são ligados a este conceito. 



$\left(-\frac{10}{1}\right.$

\section{Superfícies de ângulo constante em $\mathbb{R}^{3}$}

O presente capítulo tem como objetivo apresentar os resultados de [26] sobre as chamadas superfícies de ângulo constante no espaço $\mathbb{R}^{3}$, que são aquelas cujo campo de vetores normal unitário forma um ângulo constante com um campo de direções do espaço ambiente.

O primeiro trabalho nessa direção é [8], onde P. Carmelli e A.J. Di Scala estudam as superfícies de ângulo constante no espaço Euclidiano tridimensional, provando que as mesmas podem ser usadas para descrever interfaces que ocorrem em configurações de equilibrio de cristais líquidos. Recentemente foram publicados vários trabalhos inerentes ao estudo desta classe de superfícies em outras variedades tridimensionais (ver, por exemplo, [12, 13, 16, 23, 24, 25]). Em [8], os autores estudam as propriedades das superfícies de ângulo constante em $\mathbb{R}^{3}$, escrevendo esta condição (de ângulo constante) como uma equação de Hamilton-Jacobi que liga a superfície com o campo de direções do espaço ambiente.

No trabalho [26] os autores M.I. Munteanu e A.I. Nistor classificam completamente (com um método diferente do usado em [8]) as superfícies em $\mathbb{R}^{3}$ que formam um ângulo constante com a direção $\mathbb{R}$ obtendo o Teorema 1.2.5, que é o resultado principal deste capítulo. 


\subsection{Preliminares}

Seja $\langle.,$.$\rangle a métrica usual em \mathbb{R}^{3}$ e $\bar{\nabla}$ a sua conexão de Levi-Civita. Consideremos uma orientação em $\mathbb{R}^{3}$ e denotemos por $k$ uma direção fixada. Seja $M$ uma superfície isometricamente imersa em $\mathbb{R}^{3}$ e denotemos por $N$ a normal unitária à superfície $M$. Denotemos ainda por $\theta:=\widehat{(N, k)}$, onde $\theta \in[0, \pi)$, a função ângulo entre a normal unitária à superfície e a direção fixada. Sabemos que um vetor é tangente a $M$ se, e somente se, ele é ortogonal ao vetor normal $N$.

No Apêndice Aforam lembradas as fórmulas de Gauss e Weingarten, dadas (respectivamente) por:

$$
\begin{aligned}
& \bar{\nabla}_{X} Y=\nabla_{X} Y+h(X, Y), \\
& \bar{\nabla}_{X} N=-A(X),
\end{aligned}
$$

$X, Y \in \chi(M)$, onde $\chi(M)$ é o conjunto dos vetores tangentes a $M, \nabla$ é a conexão de Levi-Civita de $M, \operatorname{com} h$ indicamos a segunda forma fundamental de $M$ e com $A$ o operador forma. Notemos agora que, dado $Y \in \chi(M)$, de $\langle Y, N\rangle=0$, obtemos que

$$
\left\langle\bar{\nabla}_{X} Y, N\right\rangle+\left\langle Y, \bar{\nabla}_{X} N\right\rangle=0, \quad X \in \chi(M) .
$$

Logo pelas fórmulas (1.1) e (1.2), temos que

$$
\langle h(X, Y), N\rangle=\langle Y, A(X)\rangle
$$

para todo $X, Y \in \chi(M)$, onde do lado direito temos a restrição do produto escalar de $\mathbb{R}^{3}$ a $M$. Decompondo $k$ em parte normal e parte tangente, temos

$$
k=U+\cos \theta N
$$

onde $U$ é tangente a $M$ e $\|U\|=\sin \theta$. Assim, para $\theta \neq 0$, podemos definir um campo de vetores unitário por $e_{1}=\frac{U}{\|U\|}$ e considerando $e_{2}$ como sendo um campo unitário ortogonal a $e_{1}$, obtemos uma base ortonormal $\left\{e_{1}, e_{2}\right\}$ definida em cada ponto de $M$.

De agora em diante consideraremos a função ângulo $\theta$ como sendo constante.

Proposição 1.1.1. Nas hipóteses acima temos $\left[e_{1}, e_{2}\right] \| e_{2}$. 
Demonstração. Sabemos que

$$
\left[e_{1}, e_{2}\right]=\bar{\nabla}_{e_{1}} e_{2}-\bar{\nabla}_{e_{2}} e_{1} .
$$

Note que, como $\|U\|=\sin \theta$ e $e_{1}=\frac{U}{\|U\|}$, segue que $U=\sin \theta e_{1}$. Assim, podemos escrever

$$
k=\sin \theta e_{1}+\cos \theta N
$$

Derivando, então, com relação a $e_{2}$, obtemos

$$
\bar{\nabla}_{e_{2}} e_{1}=-\cot \theta \bar{\nabla}_{e_{2}} N
$$

Além disso, derivando $\left\langle N, e_{1}\right\rangle=0$ com relação a $e_{2}$, segue que

$$
\left\langle N, \bar{\nabla}_{e_{2}} e_{1}\right\rangle=-\left\langle\bar{\nabla}_{e_{2}} N, e_{1}\right\rangle
$$

Observemos que, pela fórmula (1.2), podemos escrever

$$
\bar{\nabla}_{e_{2}} N=-\rho e_{1}-\lambda e_{2}, \quad \text { onde } \quad \rho, \lambda \in C^{\infty}(M) .
$$

Substituindo a equação (1.6) na equação (1.4), obtemos

$$
\bar{\nabla}_{e_{2}} e_{1}=\cot \theta\left(\rho e_{1}+\lambda e_{2}\right)
$$

Supondo que $\theta \neq \pi / 2$ (o caso em que $\theta=\pi / 2$ será tratado separadamente) e substituindo as equações (1.7) e (1.6) em (1.5) teremos que $\rho=0$, logo

$$
\bar{\nabla}_{e_{2}} e_{1}=\lambda \cot \theta e_{2}
$$

Derivando agora (1.3) com respeito a $e_{1}$ obtemos que

$$
\bar{\nabla}_{e_{1}} e_{1}=-\cot \theta \bar{\nabla}_{e_{1}} N
$$

e, novamente pela fórmula (1.2), podemos escrever

$$
\bar{\nabla}_{e_{1}} N=-\alpha e_{1}-\beta e_{2}, \quad \alpha, \beta \in C^{\infty}(M)
$$

Sendo assim, derivando (1.3) com respeito a $e_{1}$ e combinando com (1.10), obtemos que

$$
\bar{\nabla}_{e_{1}} e_{1}=\cot \theta\left(\alpha e_{1}+\beta e_{2}\right) .
$$


Como $\left\langle\bar{\nabla}_{e_{1}} e_{1}, e_{1}\right\rangle=0$, obtemos que $\alpha=0$. Além disso, sendo que o operador $A$ é auto-adjunto, resulta

$$
\beta \cot \theta\left\langle e_{2}, e_{2}\right\rangle=\lambda \cot \theta\left\langle e_{2}, e_{1}\right\rangle=0
$$

e, de $\cot \theta \neq 0$, segue que $\beta=0$. Portanto $A\left(e_{1}\right)=0$ e, assim

$$
\bar{\nabla}_{e_{1}} e_{1}=0
$$

Observe que:

$$
\begin{aligned}
& \left\langle\bar{\nabla}_{e_{1}} e_{2}, e_{1}\right\rangle=0, \\
& \left\langle\bar{\nabla}_{e_{1}} e_{2}, e_{2}\right\rangle=0
\end{aligned}
$$

e ainda, pela fórmula de Gauss,

$$
0=\left\langle A\left(e_{1}\right), e_{2}\right\rangle=\left\langle h\left(e_{1}, e_{2}\right), N\right\rangle=\left\langle\bar{\nabla}_{e_{1}} e_{2}, N\right\rangle
$$

Sendo assim, por (1.12), (1.13) e (1.14) segue que

$$
\bar{\nabla}_{e_{1}} e_{2}=0
$$

Portanto

$$
\left[e_{1}, e_{2}\right]=\bar{\nabla}_{e_{1}} e_{2}-\bar{\nabla}_{e_{2}} e_{1}=-\bar{\nabla}_{e_{2}} e_{1}=-\lambda \cot \theta e_{2},
$$

ou seja, $\left[e_{1}, e_{2}\right] \| e_{2}$.

Temos então a seguinte:

Proposição 1.1.2. Nas condições dadas anteriormente, a conexão de Levi-Civita $\nabla$ de $M$ é dada pelas relações:

$$
\nabla_{e_{1}} e_{1}=0, \nabla_{e_{1}} e_{2}=0, \nabla_{e_{2}} e_{1}=\lambda \cot \theta e_{2} \quad e \quad \nabla_{e_{2}} e_{2}=-\lambda \cot \theta e_{1}
$$

Demonstração. Na demonstração da Proposição 1.1.1 vimos que

$$
\bar{\nabla}_{e_{1}} e_{1}=0, \quad \bar{\nabla}_{e_{1}} e_{2}=0 \quad \text { e } \quad \bar{\nabla}_{e_{2}} e_{1}=\lambda \cot \theta e_{2} .
$$

Assim, como $\nabla=(\bar{\nabla})^{\top}$, temos que:

$$
\nabla_{e_{1}} e_{1}=0, \quad \nabla_{e_{1}} e_{2}=0 \quad \text { e } \quad \nabla_{e_{2}} e_{1}=\lambda \cot \theta e_{2} .
$$


Derivando, então, $\left\langle e_{2}, e_{1}\right\rangle=0$ com relação a $e_{2}$ obtemos que

$$
\left\langle\bar{\nabla}_{e_{2}} e_{2}, e_{1}\right\rangle=-\lambda \cot \theta
$$

Como $\left\langle\bar{\nabla}_{e_{2}} e_{2}, e_{2}\right\rangle=0$, segue que

$$
\nabla_{e_{2}} e_{2}=-\lambda \cot \theta e_{1}
$$

\subsection{Caracterização das superfícies de ângulo constante}

Devido à Proposição 1.1.1, podemos escolher um sistema de coordenadas locais em $M$ da forma:

$$
r(u, v)=(x(u, v), y(u, v), z(u, v))
$$

tal que os vetores tangentes sejam $r_{u}=e_{1}$ e $r_{v} \| e_{2}$. Seja, então, $r_{v}:=\beta(u, v) e_{2}$, onde $\beta$ é uma função diferenciável em $M$. Assim podemos escrever a métrica de $M$ da forma:

$$
g=d u^{2}+\beta^{2}(u, v) d v^{2}
$$

Observação 1.2.1. No caso acima os coeficientes da primeira forma fundamental são $E=1$, $F=0$ e $G=\beta^{2}$.

Devido à Proposição 1.1.2 podemos escrever a conexão de Levi-Civita de $M$ em termos das coordenadas $u$ e $v$. Segue, então, que:

$$
\begin{gathered}
r_{u u}=0, \\
r_{u v}=\frac{\beta_{u}}{\beta} r_{v},
\end{gathered}
$$

onde $\beta$ satisfaz

$$
\beta_{u}-\beta \lambda \cot \theta=0
$$

e, por fim,

$$
r_{v v}=\frac{\beta_{v}}{\beta} r_{v}-\beta^{2} \lambda \cot \theta r_{u}+\beta^{2} \lambda N
$$

Observando agora que $N_{u}=0$ e $N_{v}=-\lambda r_{v}$, segue da equação de Codazzi que:

$$
\lambda_{u}+\lambda^{2} \cot \theta=0 .
$$


Observação 1.2.2. Como $N_{u}=0$, segue que $e=f=0$ (onde $e, f, g$ denotam os coeficientes da segunda forma fundamental). Assim, a curvatura Gaussiana de $M$ se anula ou seja, a superfície $M$ é localmente "flat".

Observação 1.2.3. Em termos da aplicação de Gauss da superfície, podemos dizer que ela fazer um ângulo constante com uma direção fixada é equivalente ao fato de que a aplicação de Gauss descreve um círculo na esfera $\mathbb{S}^{2}$. Desde que este não tem pontos interiores em $\mathbb{S}^{2}$, segue que a curvatura Gaussiana da superfície é identicamente nula.

Proposição 1.2.4. As funções $\lambda$ e $\beta$ são dadas pelas seguintes expressões:

$$
\begin{gathered}
\lambda(u, v)=\frac{\tan \theta}{u+\alpha(v)}, \\
\beta(u, v)=\varphi(v)(u+\alpha(v)),
\end{gathered}
$$

onde $\alpha, \varphi \in C^{\infty}(\mathbb{R})$, ou

$$
\begin{gathered}
\lambda(u, v)=0, \\
\beta(u, v)=\beta(v) .
\end{gathered}
$$

Demonstração. Primeiramente resolvemos a equação (1.21) e achamos a função $\lambda$. Depois substituímos na equação (1.19) e achamos a solução para $\beta$.

Iremos agora enunciar e provar o resultado principal deste capítulo, que caracteriza as superfícies de $\mathbb{R}^{3}$ que formam ângulo costante com a direção $\mathbb{R}$.

Teorema 1.2.5. (Da caracterização) Uma superfície $M$ em $\mathbb{R}^{3}$ é uma superfície de ângulo constante (com relação à direção $\mathbb{R}$ ) se, e somente se, ela é, localmente, isométrica a alguma das seguintes superfícies:

1. a superfície dada por

$$
r: M \longrightarrow \mathbb{R}^{2} \times \mathbb{R}, \quad(u, v) \longmapsto(u \cos \theta(\cos v, \sin v)+\gamma(v), u \sin \theta),
$$

onde

$$
\gamma(v)=\cos \theta\left(-\int_{0}^{v} \alpha(\tau) \sin \tau d \tau, \int_{0}^{v} \alpha(\tau) \cos \tau d \tau\right)
$$

$e \alpha \in C^{\infty}(I), I \subset \mathbb{R}$ 
2. a um aberto do plano $x \sin \theta-z \cos \theta=0$;

3. a um aberto do cilindro $\gamma \times \mathbb{R}$, onde $\gamma$ é uma curva suave em $\mathbb{R}^{2}$.

Demonstração. Primeiramente vamos mostrar que as superfícies do tipo 1, 2, e 3, são de fato de ângulo constante (com relação à direção $\mathbb{R}$ ).

No caso 2, é óbvio, pois se trata de um plano. No caso 3. $M$ é um cilindro $\gamma \times \mathbb{R}, \operatorname{logo}$ a normal à superfície será sempre paralela à normal da curva $\gamma$ de $\mathbb{R}^{2}$, onde consideramos $\mathbb{R}^{2}$ como sendo o plano ortogonal à direção fixada. Sendo assim temos que $N$ é ortogonal à direção fixada, $\operatorname{logo} \theta=\pi / 2$. No caso 1 de (1.26) temos que:

$$
r_{u}=(\cos \theta \cos v, \cos \theta \sin v, \sin \theta) \quad \text { e } \quad r_{v}=((u+\alpha(v)) \cos \theta(-\sin v, \cos v), 0)
$$

Assim $N=(-\sin \theta(\cos v, \sin v), \cos \theta)$ e como $k=(0,0,1)$, segue que o ângulo entre $N$ e $k$ é constante e igual a $\theta$.

Reciprocamente, suponhamos que $M$ seja uma superfície que faz um ângulo constante a direção fixada $k$. De (1.3), sendo $r_{u}=e_{1}$, temos que:

$$
\left\langle r_{u}, k\right\rangle=\sin \theta \quad \text { e } \quad\left\langle r_{v}, k\right\rangle=0 .
$$

Se $r(u, v)=(x(u, v), y(u, v), z(u, v))$, segue que $z(u, v)=u \sin \theta \mathrm{e}$, assim,

$$
r(u, v)=(h(u, v), u \sin \theta)
$$

onde $h(u, v)=(x(u, v), y(u, v))$. Analisaremos agora os dois casos para $\lambda$ e $\beta$ dados pela Proposição 1.2 .4

Caso I: Consideremos $\lambda$ e $\beta$ dados por (1.22) e (1.23), respectivamente. Como $r_{u u}=0$, segue que $h_{u u}=0$. Por outro lado temos que $r_{u}=\left(h_{u}(u, v), \sin \theta\right)=e_{1}$ é um vetor unitário. Logo, $\left\|h_{u}\right\|=\cos \theta$ e, portanto, temos que $h_{u}=f(v) \cos \theta$, para alguma função diferenciável $f: \mathbb{R} \longrightarrow \mathbb{R}^{2}$, com $\|f(v)\|=1, v \in \mathbb{R}$. Ou seja, $f$ é uma parametrização do círculo $\mathbb{S}^{1}$. Por integração obtemos:

$$
h(u, v)=u \cos \theta f(v)+\gamma(v),
$$


onde $\gamma$ é uma curva suave em $\mathbb{R}^{2}$. Podemos supor, sem perda de generalidade, que $f$ seja a parametrização natural de $\mathbb{S}^{1}$, isto é $f(v)=(\cos v, \sin v)$. Assim

$$
r_{v}=\left(u \cos \theta(-\sin v, \cos v)+\gamma^{\prime}(v), 0\right)
$$

Derivando, agora, a equação (1.28) com relação a $u$ e levando em consideração as equações (1.18) e (1.23), resulta

$$
r_{v}=((u+\alpha(v)) \cos \theta(-\sin v, \cos v), 0) .
$$

E, como $\beta=\left\|r_{v}\right\|$, obtemos que $\varphi(v)=\cos \theta$ é constante. Portanto, uma parametrização para $M$ pode ser dada por:

$$
r(u, v)=(u \cos \theta(\cos v, \sin v)+\gamma(v), u \sin \theta),
$$

onde a curva $\gamma$ é dada por 1.27).

Caso II: Consideremos agora $\lambda$ e $\beta$ dados por (1.24) e (1.25), respectivamente. Como $r_{u u}=0$ e $r_{u v}=0$, temos que $h_{u u}=0$ e $h_{u v}=0$, o que implica que $h_{u}$ é um vetor constante de $\mathbb{R}^{2}$ e de comprimento $\cos \theta$. Sendo assim podemos escrever $h_{u}(u, v)=\cos \theta(\cos \mu, \sin \mu)$, para algum $\mu \in \mathbb{R}$. Consequentemente,

$$
h(u, v)=u \cos \theta(\cos \mu, \sin \mu)+\gamma(v),
$$

onde $\gamma$ é uma curva suave de $\mathbb{R}^{2}$. Então,

$$
r(u, v)=(u \cos \theta(\cos \mu, \sin \mu)+\gamma(v), u \sin \theta) .
$$

Agora, como $r_{u}$ e $r_{v}$ são ortogonais, escrevendo $\gamma(v)=(x(v), y(v))$ e supondo que $\cos \mu \neq 0$, temos que:

$$
x^{\prime}(v)=-y^{\prime}(v) \frac{\sin \mu}{\cos \mu} .
$$

Portanto,

$$
\gamma(v)=\alpha(v)(-\sin \mu, \cos \mu)+c_{0}
$$

onde,

$$
\alpha(v)=\frac{y(v)}{\cos \mu} \quad \text { e } \quad c_{0} \in \mathbb{R}^{2} .
$$

Rotacionando, então, o ângulo $\mu$ no plano- $x y$ de forma que $\cos \mu=1$ e $\sin \mu=0$ obtemos:

$$
r(u, v)=(u \cos \theta, \alpha(v), u \sin \theta),
$$


a qual representa uma parametrização do plano:

$$
x \sin \theta-y \cos \theta=0 .
$$

Para completar a demonstração, resta analisar os seguintes casos para o ângulo $\theta$ :

- Se $\theta=0$, então temos que a normal $N$ coincide com a direção $k$. Como $r_{u}$ e $r_{v}$ são tangentes a $M$ teremos que $\left\langle r_{u}, k\right\rangle=0 \mathrm{e}\left\langle r_{v}, k\right\rangle=0$, assim $\langle r, k\rangle=$ constante. Essa equação determina um plano paralelo ao plano $x y$ e pode ser parametrizado por $r(u, v)=(u, v, c)$, $c \in \mathbb{R}$.

- Se $\theta=\pi / 2$, então $k$ será tangente à superfície. Nesse caso teremos que $M$ será o produto de uma curva $\gamma$ de $\mathbb{R}^{2}$ e $\mathbb{R}$, ou seja, um cilindro que pode ser parametrizado por $r(u, v)=$ $(\gamma(v), u)$.

Exemplo 1.2.6. Em todos os exemplos a seguir consideraremos $\theta=\pi / 4$.

1. Tomando $\alpha(v)=1$, temos:

$$
r(u, v)=\frac{1}{\sqrt{2}}((1+u) \cos v-1,(1+u) \sin v, u) .
$$

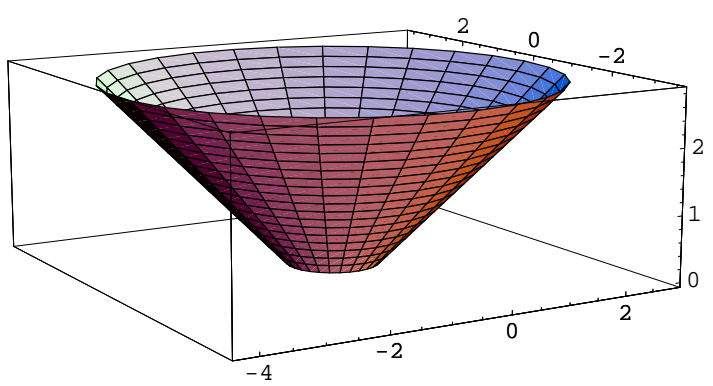

Figura 1.1: Superfície de ângulo constante $\operatorname{com} \alpha(v)=1$.

2. Tomando $\alpha(v)=v$, resulta que

$$
r(u, v)=\frac{1}{\sqrt{2}}((v+u) \cos v-\sin v,(v+u) \sin v+\cos v-1, u) .
$$

Esta superfície é representada na Figura 1.2 


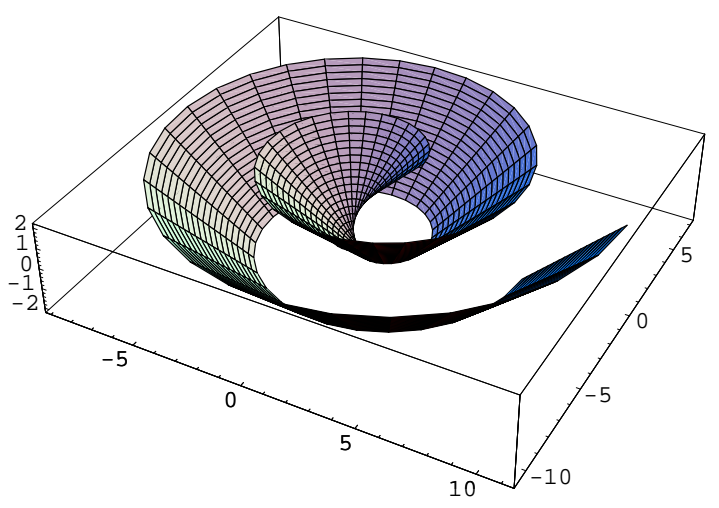

Figura 1.2: Superfície de ângulo constante com $\alpha(v)=v$.

3. Considere $\alpha(v)=\cos v$. Obtemos que

$$
r(u, v)=\frac{1}{\sqrt{2}}\left(u \cos v-\frac{\sin ^{2} v}{2}, u \sin v+\frac{v+\sin v \cos v}{2}, u\right)
$$

veja a Figura 1.3 .

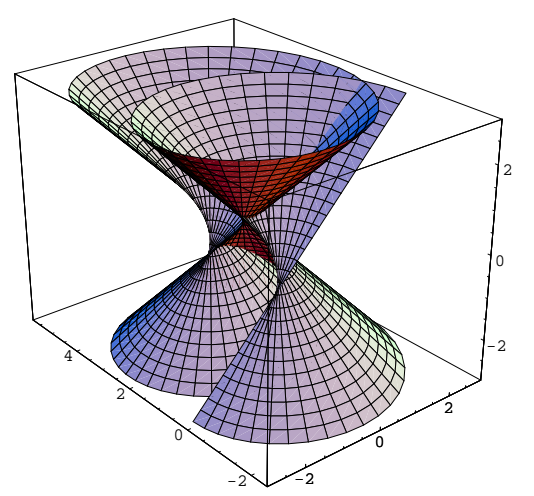

Figura 1.3: Superfície de ângulo constante $\operatorname{com} \alpha(v)=\cos v$.

4. Se $\alpha(v)=2 \sin v$, temos que

$$
r(u, v)=\frac{1}{\sqrt{2}}\left(u \cos v-v+\cos v \sin v, u \sin v+\sin ^{2} v, u\right) .
$$

veja a Figura 1.4 


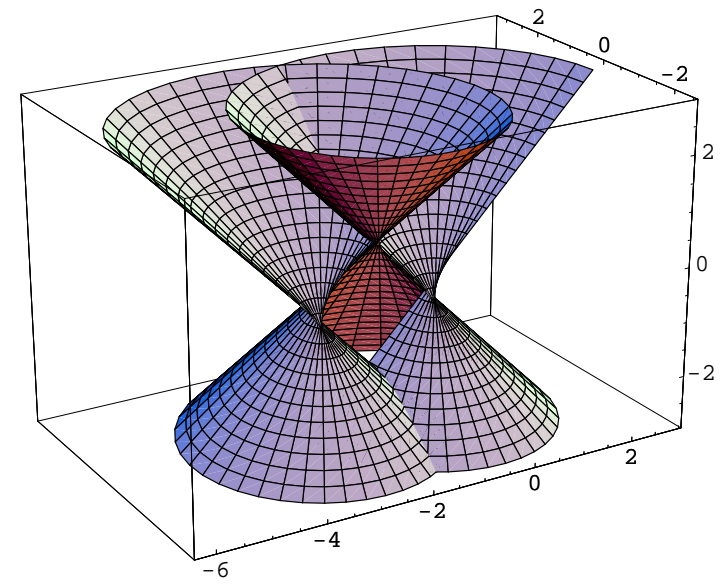

Figura 1.4: Superfície de ângulo constante $\operatorname{com} \alpha(v)=2 \sin v$.

Vamos terminar o capítulo com o seguinte resultado:

Proposição 1.2.7. 1. As únicas superfícies mínimas de ângulo constante (com relação à direção $\mathbb{R})$ em $\mathbb{R}^{3}$ são os planos que fazem um ângulo $\theta$ com a direção fixada $k$.

2. As superfícies de ângulo constante (com relação à direção $\mathbb{R}$ ) em $\mathbb{R}^{3}$ com curvatura média constante e não nula são os cilindros.

Demonstração. A curvatura média de uma superfície é dada pela fórmula

$$
H=\frac{1}{2} \frac{e G-2 f F+g E}{E G-F^{2}} .
$$

Assim, dada uma superfície $M$ de ângulo constante $\theta$, segue das Observações 1.2.1 e 1.2.2 que:

$$
H(u, v)=\frac{g}{2 \beta^{2}(u, v)} .
$$

De

$$
r_{v v}=\frac{\beta_{u}}{\beta} r_{v}-\beta^{2} \lambda \cot \theta r_{u}+\beta^{2} \lambda N
$$

temos que $g=\beta^{2} \lambda$ e, assim, $H=\lambda / 2$. Portanto $H=0$ se, e somente se, $\lambda=0$. Logo, pelo Teorema 1.2.5, teremos que $M$ será um plano que faz ângulo constante com a direção fixada. Agora, se $M$ é uma superfície de curvatura média constante e não nula, teremos que:

$$
H=\frac{\lambda}{2}=\text { constante }, \quad \lambda \neq 0 .
$$

Como $\lambda$ satisfaz a equação (1.21) obtemos que $\theta=\pi / 2 \mathrm{e}$, pelo Teorema1.2.5, temos que $M$ é um cilindro. 



\begin{tabular}{l|l|}
\hline CAPÍtulo \\
\cline { 2 - 2 } \\
2 \\
\hline
\end{tabular}

\section{Curvas e superfícies em $\mathbb{R}^{3}$ que fazem ângulo constante com campos de}

Killing

O presente capítulo tem como objetivo apresentar os resultados de [27] sobre a classificação das curvas e das superfícies que fazem ângulo constante com certos campos de Killing em $\mathbb{R}^{3}$.

Um resultado importante e conhecido da geometria diferencial das curvas em $\mathbb{R}^{3}$, que foi enunciado por M.A. Lancret em 1802 e provado por B. de Saint Venant em 1845 (veja [32] para maiores detalhes), afirma que uma curva forma um ângulo constante com respeito a um campo de Killing unitário de $\mathbb{R}^{3}$ se, e somente se, a razão entre sua torção $\tau$ e a sua curvatura $k$ é constante. Curvas deste tipo são chamadas hélices generalizadas. Se as funções $\tau$ e $k$ são ambas constantes e não nulas, então as curvas são ditas hélices circulares. O problema de Lancret-de Saint Venant foi generalizado para curvas em outras variedades de dimensão três como, por exemplo, as formas espaciais (ver [2]). 
18 Capítulo 2 - Curvas e superfícies em $\mathbb{R}^{3}$ que fazem ângulo constante com campos de Killing

No trabalho [26] os autores M.I. Munteanu e A.I. Nistor classificam as curvas planas e espaciais de $\mathbb{R}^{3}$ que fazem ângulo constante com o campo de Killing $V=-y \partial_{x}+x \partial_{y}$, obtendo os Teoremas 2.1.1 e 2.1.3 (respectivamente).

Passando das curvas para as superfícies, um problema natural é o estudo de superfícies em espaços tridimensionais que fazem ângulo constante com certos campos de vetores, do qual tratamos no Capítulo 1 para o caso em que o campo é $\partial_{z}$.

O resultado mais importante deste capítulo é o Teorema 2.2.2, que nos da uma classificação completa das superfícies cuja normal unitária faz ângulo constante com o campo de Killing $V=-y \partial_{x}+x \partial_{y}$, a qual inclui: semi-planos tendo o eixo $z$ como fronteira, superfícies de revolução ao redor do eixo $z$, cilindros retos sobre espirais logarítmicas e a superfície de Dini.

\subsection{Alguns resultados sobre curvas em $\mathbb{R}^{3}$}

Nesta seção mostraremos algumas propriedades de curvas planas e espaciais do espaço Euclidiano $\mathbb{R}^{3}$ envolvendo o ângulo entre duas curvas. Como os resultados são locais podemos considerar duas curvas $\gamma$ e $\tilde{\gamma}$ sem auto-intersecção e parametrizadas pelo mesmo parâmetro $t \in I \subset \mathbb{R}$.

Dado o ponto $t \in I$, definiremos o ângulo $\theta$ (em $t$ ) entre $\gamma$ e $\tilde{\gamma}$ como sendo o ângulo entre os vetores tangentes $\gamma^{\prime}(t)$ e $\tilde{\gamma}^{\prime}(t)$. Iremos considerar o caso em que a função $\theta(t)$ é constante. Consideremos, primeiramente, duas curvas planas que estão no mesmo plano. Se uma das curvas for uma linha reta e a outra curva faz um ângulo constante com esta, então esta será também uma linha reta.

Agora vamos considerar o caso de uma curva $\gamma$ que faz um ângulo constante com o círculo unitário $\mathbb{S}^{1}$. Tomemos

$$
\gamma: I \subset \mathbb{R} \longrightarrow \mathbb{R}^{2}, \quad \gamma(s)=\left(\gamma_{1}(s), \gamma_{2}(s)\right)
$$

parametrizada pelo comprimento de arco, e o círculo unitário $\mathbb{S}^{1}$ parametrizado pelo mesmo parâmetro $s$, ou seja

$$
C(s)=(\cos \sigma(s), \sin \sigma(s))
$$


onde $\sigma$ é uma função diferenciável definida em $I$. Observemos que, em geral, o parâmetro do comprimento de arco de duas curvas não é o mesmo. Sejam

$$
t_{C}=(-\sin \sigma(s), \cos \sigma(s)) \quad \text { e } \quad t_{\gamma}=\left(\gamma_{1}^{\prime}(s), \gamma_{2}^{\prime}(s)\right)
$$

os vetores tangentes unitários das curvas $C$ e $\gamma$, respectivamente. O ângulo entre essas duas curvas $\angle\left(t_{C}, t_{\gamma}\right)$ será uma constante $\theta \in[0, \pi)$. Assim

$$
\cos \theta=-\sin \sigma(s) \gamma_{1}^{\prime}(s)+\cos \sigma(s) \gamma_{2}^{\prime}(s)
$$

Notemos agora que, como $\left\|t_{\gamma}\right\|=1$, podemos escrever:

$$
t_{\gamma}=(\cos \omega(s), \sin \omega(s)), \quad \text { onde } \quad \omega(s)=\pi-(\theta+\sigma(s)) .
$$

Sendo assim, uma parametrização para a curva $\gamma$ é dada por

$$
\begin{aligned}
\gamma(s)= & \left(\sin \theta \int \cos \sigma(s) d s-\cos \theta \int \sin \sigma(s) d s,\right. \\
& \left.\cos \theta \int \cos \sigma(s) d s+\sin \theta \int \sin \sigma(s) d s\right),
\end{aligned}
$$

após uma translação em $\mathbb{R}^{2}$. Se denotarmos por

$$
\gamma_{0}(s)=\left(-\int \sin \sigma(s) d s, \int \cos \sigma(s) d s\right),
$$

então a parametrização (2.1) pode ser escrita como:

$$
\gamma(s)=(\cos \theta-i \sin \theta) \gamma_{0}(s), \quad i=\sqrt{-1} .
$$

Para obtermos uma interpretação geométrica da fórmula acima, consideremos $\theta=\pi / 3$ e $\sigma(s)=s^{2}$, com $s \in[-\pi, \pi]$. Se a curva $\gamma_{0}$ (verde) faz um ângulo constante $\theta=0$ com o círculo $\mathbb{S}^{1}$ (rosa), então a curva $\gamma$ (azul) representa uma rotação de um ângulo $\pi / 3$ de $\gamma_{0}$ no sentido horário (veja a Figura 2.1).

Do mesmo modo, podemos analisar o caso das curvas espaciais. A primeira questão surge quando queremos encontrar as curvas espaciais que fazem um ângulo constante com uma linha reta. Um resultado clássico nos diz que, neste caso, a curva é uma hélice. Sem perda de generalidade, essa curva pode ser tomada como sendo paralela a um dos eixos coordenados, que seria uma 


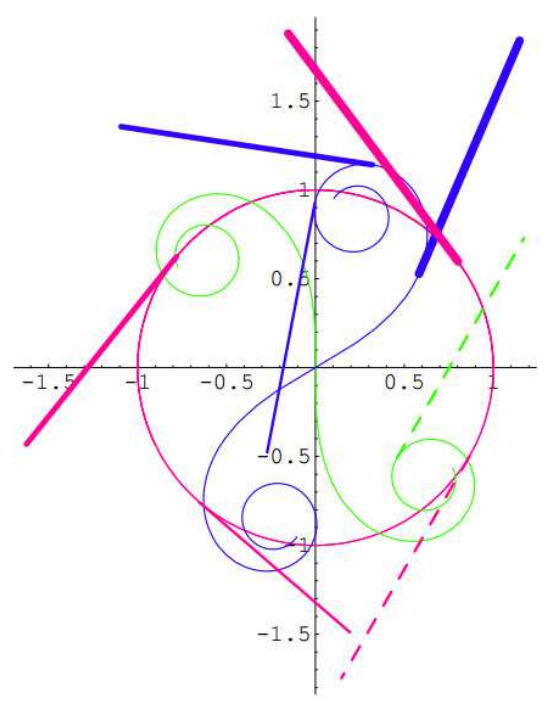

Figura 2.1

curva integral de um dos campos de Killing em $\mathbb{R}^{3}$. Motivados por essa observação, gostaríamos de encontrar quais curvas fazem um ângulo constante com um campo de Killing em $\mathbb{R}^{3}$.

Um campo vetorial $V$ de $\mathbb{R}^{3}$ é um campo de Killing se, e somente se, satisfaz a equação:

$$
\left\langle\bar{\nabla}_{X} V, Y\right\rangle+\left\langle\bar{\nabla}_{Y} V, X\right\rangle=0
$$

onde $X, Y$ são campos de vetores em $\mathbb{R}^{3}$ e $\bar{\nabla}$ é a conexão de Levi-Civita relativa à métrica usual $\langle.,$.$\rangle de \mathbb{R}^{3}$ (veja Apêndice $\mathrm{A}$ ). Além disso, provamos que o conjunto de soluções para essa equação é:

$$
\left\{\partial_{x}, \partial_{y}, \partial_{z}, x \partial_{y}-y \partial_{x}, y \partial_{z}-z \partial_{y}, z \partial_{x}-x \partial_{z}\right\}
$$

e nos dá uma base para os campos de Killing de $\mathbb{R}^{3}$.

Já vimos que, curvas espaciais que fazem um ângulo constante com os campos de Killing $\partial_{x}, \partial_{y}$ ou $\partial_{z}$ são hélices. Vamos agora encontrar todas as curvas que fazem um ângulo constante com o campo de Killing das rotações ao redor do eixo $z$ :

$$
V=-y \partial_{x}+x \partial_{y}
$$

Note que o problema para os dois outros campos restantes é análogo. Consideremos, então, uma curva plana $\gamma$, no plano- $x y$, dada por $\gamma(s)=\left(\gamma_{1}(s), \gamma_{2}(s)\right), s \in I \subset \mathbb{R}$, parametrizada pelo 
comprimento de arco, e suponhamos que ela faça um ângulo constante $\theta$ com o campo $V$. Sendo que o vetor tangente a $\gamma$ é dado por $t_{\gamma}=\left(\gamma_{1}^{\prime}, \gamma_{2}^{\prime}\right)$ e $V_{\mid \gamma}=\left(-\gamma_{2}, \gamma_{1}\right)$, a condição $\angle\left(t_{\gamma}, V_{\mid \gamma}\right)=\theta$ pode ser reescrita como:

$$
\sqrt{\gamma_{1}^{2}(s)+\gamma_{2}^{2}(s)} \cos \theta=-\gamma_{1}^{\prime}(s) \gamma_{2}(s)+\gamma_{2}^{\prime}(s) \gamma_{1}(s)
$$

Supondo que $\gamma$ esteja parametrizada em coordenadas polares, isto é:

$$
\gamma(s)=(r(s) \cos \phi(s), r(s) \sin \phi(s))
$$

temos que a equação (2.2) se torna

$$
\cos \theta=r(s) \phi^{\prime}(s)
$$

Também, como $\gamma$ é parametrizada pelo comprimento de arco, temos que

$$
r^{\prime}(s)^{2}+r(s)^{2} \phi^{\prime}(s)^{2}=1 .
$$

Combinando as equações (2.2) e (2.4) temos que considerar os casos que seguem:

- $\operatorname{Se} \theta=0$, então

$$
r(s)=r_{0}>0 \quad \text { e } \quad \phi(s)=\frac{1}{r_{0}} s+\phi_{0}, \quad r_{0}, \phi_{0} \in \mathbb{R} .
$$

Logo

$$
\gamma(s)=\left(r_{0} \cos \left(\frac{s}{r_{0}}+\phi_{0}\right), r_{0} \sin \left(\frac{s}{r_{0}}+\phi_{0}\right)\right)
$$

é um círculo de centro na origem e raio $r_{0}$.

- $\operatorname{Se} \theta \neq 0$, temos

$$
r(s)=s \sin \theta+s_{0},
$$

$\log 0$

$$
\phi(s)=\cot \theta \ln \left(s \sin \theta+s_{0}\right)+\phi_{0},
$$

onde $s_{0}, \phi_{0} \in \mathbb{R}$. Assim, resulta que

$$
\begin{aligned}
\gamma(s)= & \left(\left(s \sin \theta+r_{0}\right) \cos \left(\cot \theta \ln \left(s \sin \theta+s_{0}\right)+\phi_{0}\right),\right. \\
& \left.\left(s \sin \theta+r_{0}\right) \sin \left(\cot \theta \ln \left(s \sin \theta+s_{0}\right)+\phi_{0}\right)\right) .
\end{aligned}
$$




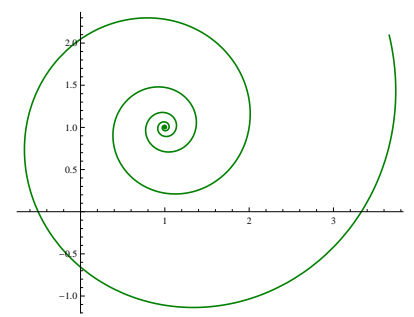

Figura 2.2: Curva fazendo ângulo constante $\theta \neq 0$ com o campo de Killing $V$.

Temos, então, o seguinte resultado:

Teorema 2.1.1. Seja $\gamma$ uma curva plana de $\mathbb{R}^{3}$ contida no plano-xy que faz ângulo constante $\theta$ com o campo de Killing $V=-y \partial_{x}+x \partial_{y}$. Então $\gamma$ é uma das seguintes curvas:

1. um círculo centrado na origem e raio $r_{0}$;

2. uma linha reta passando pela origem;

3. a espiral logarítmica $r(\phi)=e^{\tan \theta\left(\phi-\phi_{0}\right)}$.

Demonstração. As demonstrações de 1, e 3, derivam das contas feitas anteriormente. Quanto à verificação de 2, a mesma pode ser feita tomando $\theta=\pi / 2 \mathrm{em}(2.5)$.

Observação 2.1.2. Note que, o resultado acima não é de fato surpreendente, desde que o círculo $\mathbb{S}^{1}\left(r_{0}\right)$ é uma curva integral de $V$ e a espiral logarítmica, também conhecida como espiral equitangular, é caracterizada pela propriedade de que o ângulo entre seu vetor tangente e o radial é constante.

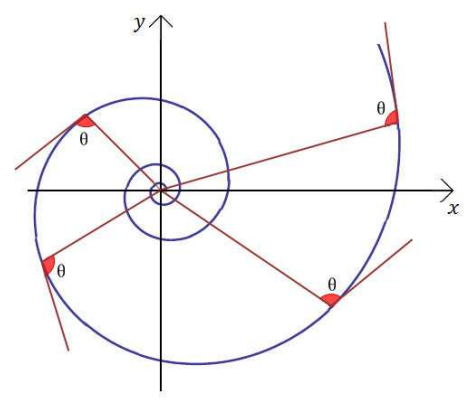

Figura 2.3: O ângulo $\theta$ entre o vetor tangente e o radial é igual em todo ponto da curva. 
Suponhamos agora que $\gamma$ seja uma curva espacial, parametrizada pelo comprimento de arco, que faça ângulo constante com o campo de Killing $V$. Assim, se $\gamma: I \subset \mathbb{R} \longrightarrow \mathbb{R}^{3}$ é parametrizada por:

$$
\gamma(s)=\left(\gamma_{1}(s), \gamma_{2}(s), \gamma_{3}(s)\right)
$$

em coordenadas cilíndricas obtemos

$$
\gamma(s)=(r(s) \cos \phi(s), r(s) \sin \phi(s), z(s))
$$

Como $t_{\gamma}=\left(\gamma_{1}^{\prime}, \gamma_{2}^{\prime}, \gamma_{3}^{\prime}\right)$ e $V_{\mid \gamma}=\left(-\gamma_{2}, \gamma_{1}, 0\right)$, a condição de que $\angle\left(t_{\gamma, V_{\mid \gamma}}\right)=\theta$ nos da novamente que:

$$
r(s) \phi^{\prime}(s)=\cos \theta .
$$

Como $\gamma$ é parametrizada pelo comprimento de arco, resulta que

$$
r^{\prime}(s)^{2}+z^{\prime}(s)^{2}=\sin ^{2} \theta
$$

ou seja $s \mapsto\left(r^{\prime}(s), z^{\prime}(s)\right)$ é uma parametrização de uma circunferência de raio $\sin \theta$. Logo, existe uma função $\omega$ tal que

$$
r^{\prime}(s)=\sin \theta \cos \omega(s) \quad \text { e } \quad z^{\prime}(s)=\sin \theta \sin \omega(s) .
$$

Assim, a menos de translações e rotações em torno do eixo $z$, temos:

$$
\begin{gathered}
r(s)=\sin \theta \int_{0}^{s} \cos \omega(\zeta) d \zeta+r_{0}, \\
z(s)=\sin \theta \int_{0}^{s} \sin \omega(\zeta) d \zeta
\end{gathered}
$$

e

$$
\phi(s)=\cos \theta \int_{0}^{s} \frac{1}{r(\zeta)} d \zeta
$$

onde $r_{0} \in \mathbb{R}$ e $\omega$ é uma função suave definida em $I \subset \mathbb{R}$.

Temos, então, o seguinte

Teorema 2.1.3. Seja $\gamma$ uma curva espacial contida em $\mathbb{R}^{3} \backslash O z$ a qual faz um ângulo constante $\theta$ com o campo de Killing $V=-y \partial_{x}+x \partial_{y}$. Então em coordenadas cilíndricas, a menos de translações e rotações em torno do eixo $z$, a curva $\gamma$ é dada pelas equações (2.8), (2.9) e (2.10). 
A seguir vamos considerar alguns exemplos $\operatorname{com} \theta \neq 0, \pi / 2$ e $r_{0}=0$.

Exemplo 2.1.4. Se $\omega(s)=\omega_{0}$ é constante, temos

$$
\phi(s)=\frac{\cot \theta}{\cos \omega_{0}} \ln (s) .
$$

Então,

- se $\omega_{0}=0$, segue que $r(s)=s \sin \theta, z(s)=0$ e $\phi(s)=\cot \theta \ln s$. Assim:

$$
\gamma(s)=(s \sin \theta \cos (\cot \theta \ln s), s \sin \theta \sin (\cot \theta \ln s), 0),
$$

ou seja, $\gamma$ é a curva plana dada pela espiral logarítmica

$$
r(s)=\sin \theta e^{\tan \theta \phi(s)} .
$$

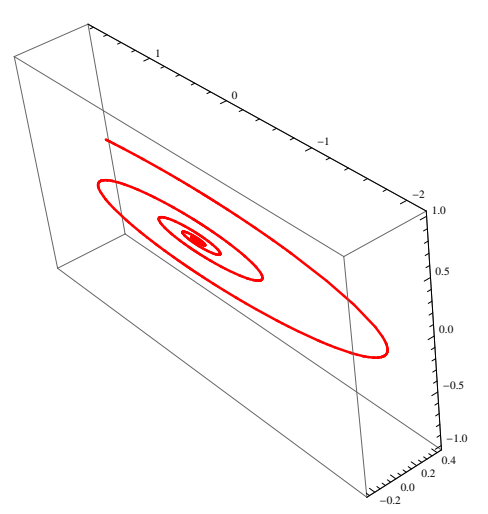

Figura 2.4: Curva de $\mathbb{R}^{3}$ que faz ângulo constante com o campo $V$ com $\omega_{0}=0$.

- Se $\omega_{0} \neq 0$, então

$$
\cot ^{2} \omega_{0} z(s)^{2}=r(s)^{2} \cos ^{2} \phi(s)+r(s)^{2} \sin ^{2} \phi(s)=\gamma_{1}(s)^{2}+\gamma_{2}(s)^{2},
$$

ou seja, o traço de $\gamma$ está contido no cone $x^{2}+y^{2}-\cot ^{2} \omega_{0} z^{2}=0$. Além disso, a projeção de $\gamma$ no plano- $x y$ é dada por:

$$
\operatorname{proj}_{x y}(\gamma)=\left(\sin \theta \cos \omega_{0} \cos \phi(s), \sin \theta \cos \omega_{0} \sin \phi(s)\right),
$$

isto é, a espiral logarítmica

$$
r(s)=\sin \theta \cos \omega_{0} e^{\left(\cos \omega_{0} \tan \theta\right) \phi} .
$$



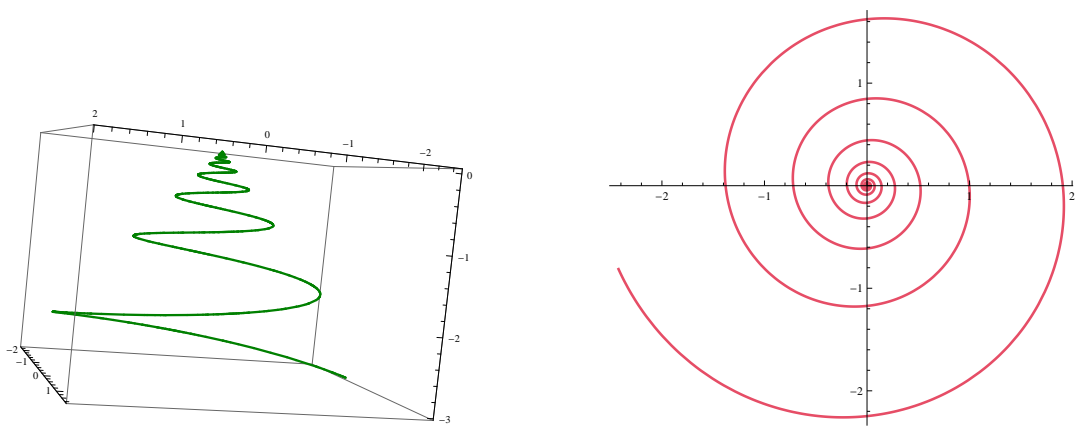

Figura 2.5: Curva de $\mathbb{R}^{3}$ que faz ângulo constante com o campo $V$, com $\omega_{0} \neq 0$, e a sua projeção no plano $x y$.

Exemplo 2.1.5. Se $\omega$ é uma função afim e não constante, isto é, $\omega(s)=m s+n, m, n \in \mathbb{R}$, $m \neq 0$, temos

$$
r(s)=\frac{\sin \theta}{m} \sin (m \zeta+n), \quad z(s)=-\frac{\sin \theta}{m} \cos (m \zeta+n)
$$

$\mathrm{e}$

$$
\phi(s)=\cot \theta\left(\ln \left[2 \sin \left(\frac{m s+n}{2}\right)\right]-\ln \left[2 \cos \left(\frac{m s+n}{2}\right)\right]\right)
$$

isto é, $\gamma$ é a espiral logarítmica

$$
r(\phi)=\frac{\sin \theta}{m \cosh (\phi \tan \theta)}
$$
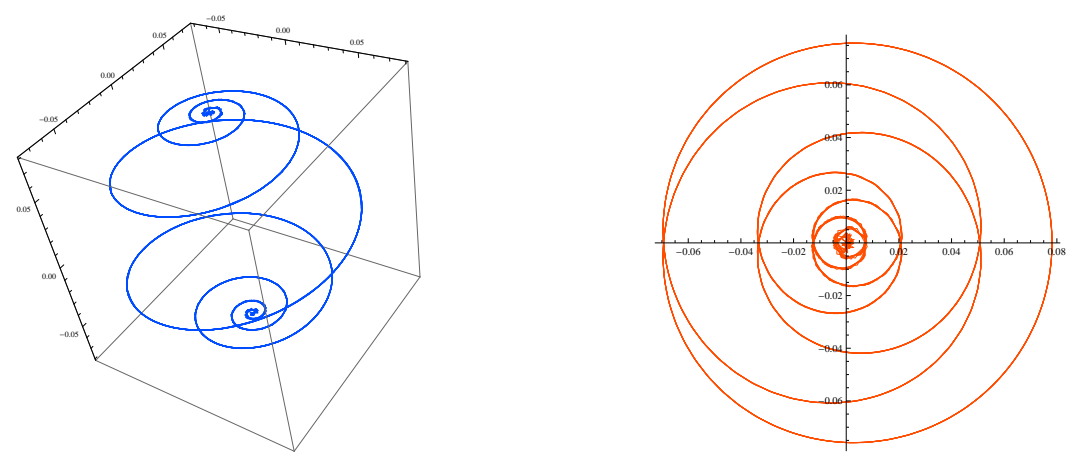

Figura 2.6: Curva que faz ângulo constante com $V$ obtida tomando $\omega(s)=2 s+1$ e a sua projeção no plano $x y$. 
Exemplo 2.1.6. $\operatorname{Se} \omega(s)=\arccos (s)$, teremos que

$$
r(s)=\frac{s^{2}}{2} \sin \theta, \quad z(s)=\sin \theta \int_{0}^{s} \sin (\arccos \zeta) d \zeta
$$

$\mathrm{e}$

$$
\phi=-2 \frac{\cot \theta}{s} .
$$

Logo $\gamma$ é dada pela espiral logarítmica

$$
r(\phi)=2 \frac{\sin \theta \cot ^{2} \theta}{\phi^{2}} .
$$

Veja a Figura 2.7

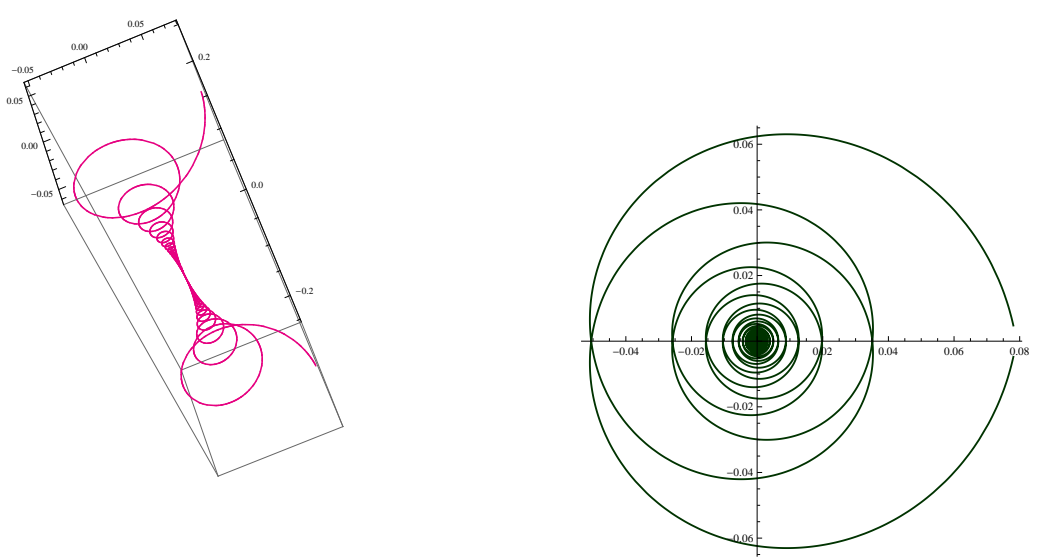

Figura 2.7: Curva que faz ângulo constante $\operatorname{com} V, \operatorname{com} \omega(s)=\arccos (s)$, e a sua projeção no plano $x y$.

\subsection{Classificação das superfícies que fazem ângulo cons- tante com $V$}

Nessa seção estaremos interessados em classificar todas as superfícies do espaço $\mathbb{R}^{3}$ que fazem ângulo constante com o campo de Killing $V$. Observemos que, neste caso, devemos ter que $V=-y \partial_{x}+x \partial_{y}$ seja não nulo em todo ponto da superfície. Sendo assim, a superfície deve estar contida em $\mathbb{R}^{3} \backslash O z$. 
Uma motivação importante para esse estudo é o caso particular em que $\theta=\pi / 2$. Seja $M$ uma superfície dada como gráfico:

$$
F: D \subset \mathbb{R}^{2} \longrightarrow \mathbb{R}^{3}, \quad F(x, y)=(x, y, f(x, y)) .
$$

Impondo que $V$ seja tangente à superfície $M$, temos que, dado $p \in M, V(p) \in T_{p} M$, ou seja, $V(p)$ deve ser uma combinação linear dos vetores $\left(1,0, f_{x}\right)$ e $\left(0,1, f_{y}\right)$. Como $V(x, y, z)=(-y, x, 0)$, temos que $f$ deve satisfazer $y f_{x}-x f_{y}=0$, ou seja:

$$
f(x, y)=g\left(\sqrt{x^{2}+y^{2}}\right)
$$

para alguma função real $g$ diferenciável, i.e. $M$ é uma superfície de rotação ao redor do eixo $z$. Observe que no caso particular em que $\theta=0$, obtemos semi-planos tendo o eixo $O z$ como fronteira.

Vamos agora considerar o caso geral em que $\theta \neq 0, \pi / 2$. Seja $g$ a métrica de $M, \nabla$ a conexão de Levi-Civita associada a ela e $\bar{\nabla}$ a conexão de Levi-Civita de $\mathbb{R}^{3}$. A partir das fórmulas (1.1) e (1.2), temos que se $h$ é a segunda forma fundamental da superfície e $A$ é o operador forma associado a ele, então

$$
\langle h(X, Y), N\rangle=g(X, A(Y)),
$$

para todo $X, Y \in \chi(M)$. Considerando o campo $V$ restrito aos pontos de $M$, podemos decompôlo da forma:

$$
V=T+\mu \cos \theta N
$$

onde $\mu=\|V\|$ e $T$ é a componente de $V$ em $T M$. Observe que neste caso $\|T\|=\mu \sin \theta$. Tomando $e_{1}=\frac{T}{\|T\|}$, podemos então escolher um campo unitário $e_{2} \in \chi(M)$ de forma que $\left\{e_{1}, e_{2}\right\}$ forme uma base ortonormal de $T M$. Desta forma:

$$
V=\mu \sin \theta e_{1}+\mu \cos \theta N
$$

Temos ainda que, tomando um campo $X \in \chi\left(\mathbb{R}^{3}\right)$ arbitrário, podemos escrever

$$
\bar{\nabla}_{X} V=k \times X
$$

onde $k=(0,0,1)$ e " $\times$ " representa o produto vetorial em $\mathbb{R}^{3}$. Além disso, se $X \in \chi(M)$ então, resulta que

$$
\bar{\nabla}_{X} V=X(\mu)\left(\sin \theta e_{1}+\cos \theta N\right)+\mu \sin \theta\left(\nabla_{X} e_{1}+h\left(X, e_{1}\right)\right)-\mu \cos \theta A(X) .
$$


28 Capítulo 2 - Curvas e superfícies em $\mathbb{R}^{3}$ que fazem ângulo constante com campos de Killing

Como $e_{1}$ e $e_{2}$ são tangentes a $M$, podemos decompor $k \times e_{1}$ e $k \times e_{2}$ na base $\left\{e_{1}, e_{2}, N\right\}$. Considerando os seguintes ângulos:

$$
\angle(N, k)=\varphi, \quad \angle\left(e_{1}, k\right)=\eta \quad \mathrm{e} \quad \angle\left(e_{2}, k\right)=\psi,
$$

temos que

$$
k=\cos \eta e_{1}+\cos \psi e_{2}+\cos \varphi N
$$

e, como $\|k\|=1$, segue que

$$
\cos \eta=\cos \xi \sin \psi \quad \text { e } \quad \cos \varphi=\sin \xi \sin \psi
$$

para algum $\xi \in \mathbb{R}$. Além disso, como $V$ está contido no plano- $x y$ temos que $\langle V, k\rangle=0$. Portanto, como $\theta \neq 0$, segue que:

$$
\frac{\cos \eta}{\cos \varphi}=-\frac{\cos \theta}{\sin \theta}
$$

Combinando as equações (2.14) e (2.15) obtemos que $\xi=-\theta$ e, assim

$$
\cos \varphi=-\sin \theta \sin \psi \quad \text { e } \quad \cos \eta=\cos \theta \sin \psi
$$

Nessas notações temos:

$$
k \times e_{1}=-\sin \theta \sin \psi e_{2}-\cos \psi N, \quad k \times e_{2}=\sin \theta \sin \psi e_{1}+\cos \theta \sin \psi N .
$$

Além disso, considerando $X=e_{1}$ e, depois, $X=e_{2}$ em (2.12) e (2.13) e igualando à equação (2.17), obtemos que

$$
\begin{gathered}
e_{1}(\mu)=-\cos \theta \cos \psi, \\
e_{2}(\mu)=\sin \psi .
\end{gathered}
$$

Como consequência, a matriz do operador forma associado a segunda forma fundamental será dada por

$$
A=\left(\begin{array}{cc}
-\frac{\sin \theta \cos \psi}{\mu} & 0 \\
0 & \lambda
\end{array}\right),
$$

onde $\lambda$ é uma aplicação diferenciável em $M$. Segue ainda que $e_{1}$ e $e_{2}$ são as direções principais de $M$. A partir das equações obtidas até agora, temos que a conexão de Levi-Civita de $g$ em termos da base $\left\{e_{1}, e_{2}, N\right\}$ é dada por

$$
\begin{array}{ll}
\nabla_{e_{1}} e_{1}=-\frac{\sin \psi}{\mu} e_{2}, & \nabla_{e_{1}} e_{2}=\frac{\sin \psi}{\mu} e_{1}, \\
\nabla_{e_{2}} e_{1}=\lambda \cot \theta e_{2}, & \nabla_{e_{2}} e_{2}=-\lambda \cot \theta e_{1} .
\end{array}
$$


Portanto, o colchete de Lie de $e_{1}$ e $e_{2}$ é dado por:

$$
\left[e_{1}, e_{2}\right]=\frac{\sin \psi}{\mu} e_{1}-\lambda \cot \theta e_{2}
$$

Calculando $\left[e_{1}, e_{2}\right](\mu)$ de duas formas, usando a definição e a simetria, e levando em consideração as equações (2.18) e (2.19), obtemos que

$$
-\cos \psi e_{1}(\psi)+\cos \theta \sin \psi e_{2}(\psi)=\frac{\cos \theta \sin \psi \cos \psi}{\mu}+\lambda \cot \theta \sin \psi .
$$

De agora em diante usaremos coordenadas cilíndricas, de forma que a parametrização da superfície $M$ possa ser escrita na forma

$$
F: D \subset \mathbb{R}^{2} \longrightarrow \mathbb{R}^{3} ; \quad(u, v) \longmapsto(r(u, v), \phi(u, v), z(u, v)) .
$$

Nessas coordenadas a métrica de $\mathbb{R}^{3}$ se torna $\langle\rangle=,d r^{2}+d z^{2}+r^{2} d \phi^{2}$ e sua conexão de Levi-Civita é dada por:

$$
\begin{array}{lll}
\bar{\nabla}_{\partial_{r} \partial_{r}}=0, & \bar{\nabla}_{\partial_{r} \partial_{\phi}}=\bar{\nabla}_{\partial_{\phi} \partial_{r}}=\frac{1}{r} \partial_{\phi}, & \bar{\nabla}_{\partial_{\phi} \partial_{\phi}}=-r \partial_{r}, \\
\bar{\nabla}_{\partial_{z} \partial_{z}}=0, & \bar{\nabla}_{\partial_{z} \partial_{\phi}}=\bar{\nabla}_{\partial_{\phi} \partial_{z}}=0, & \bar{\nabla}_{\partial_{r}} \partial_{z}=\bar{\nabla}_{\partial_{z}} \partial_{r}=0 .
\end{array}
$$

Além disso, o campo de Killing $V=\partial_{\phi} \mathrm{e}\|V\|=\mu=r$. Logo, podemos escrever

$$
\begin{gathered}
\partial_{\phi}=r \sin \phi e_{1}+r \cos \phi N \\
\partial_{z}=\cos \phi \sin \psi e_{1}+\cos \psi e_{2}-\sin \theta \sin \psi N
\end{gathered}
$$

$\mathrm{e}$

$$
\partial_{r}=-\cos \theta \cos \psi e_{1}+\sin \psi e_{2}-\sin \theta \cos \psi N
$$

Assim a base $\left\{e_{1}, e_{2}, N\right\}$ pode ser expressa (em termos das novas coordenadas) como

$$
\begin{aligned}
& e_{1}=-\cos \theta \cos \psi \partial_{r}+\frac{\sin \theta}{\mu} \partial_{\phi}+\cos \theta \sin \psi \partial_{z}, \\
& e_{2}=\sin \psi \partial_{r}+\cos \psi \partial_{z}, \\
& N=\sin \theta \cos \psi \partial_{r}+\frac{\cos \theta}{\mu} \partial_{\phi}-\sin \theta \sin \psi \partial_{z} .
\end{aligned}
$$

Além disso, como $\left[e_{1}, e_{2}\right]$ pertence ao espaço gerado por $\left\{e_{1}, e_{2}\right\}$, obtemos

$$
\frac{\cos \theta \sin \psi}{\mu}+e_{1}(\psi)=0 .
$$


30 Capítulo 2 - Curvas e superfícies em $\mathbb{R}^{3}$ que fazem ângulo constante com campos de Killing

Observação 2.2.1. Se $\psi$ é constante, então teremos que $e_{1}(\psi)=0$ e, consequentemente, $\sin \psi=0$. Assim, daqui em diante consideraremos $\psi \neq 0$ e o caso $\psi=0$ será tratado separadamente.

Escrevendo a equação (2.27) na forma:

$$
\frac{e_{1}(\psi)}{\sin \psi}=-\frac{\cos \theta}{r}
$$

e tomando a derivada de (2.28) com respeito a $e_{1}$, obtemos que

$$
e_{1}\left(e_{1}(\psi)\right)=0
$$

A partir de agora nosso objetivo é encontrar, localmente, coordenadas apropriadas para a superfície de modo que consigamos escrever explicitamente as equações paramétricas de $M$ em $\mathbb{R}^{3}$. Vamos escolher, então, a coordenada local $u$ de forma que $e_{1}=\partial_{u}$. Integrando (2.29), temos que:

$$
\psi(u, v)=f(v) u+g(v),
$$

onde $g, f \in C^{\infty}(M)$. Escolhendo agora a coordenada $v$ de forma que $\psi_{v}=0$, obtemos que

$$
\psi(u, v)=c u+\tilde{c}, \quad c, \tilde{c} \in \mathbb{R}, c \neq 0
$$

Além disso, após uma translação na coordenada $u$, temos

$$
\psi(u, v)=c u, \quad c \in \mathbb{R}-\{0\} .
$$

Substituindo então (2.31) em 2.27), obtemos

$$
\mu=-\frac{\cos \theta \sin (c u)}{c} .
$$

Também, como $e_{2} \in T M$, podemos expressar $e_{2}$ em termos de $\partial_{u}$ e $\partial_{v}$ na forma:

$$
e_{2}=a(u, v) \partial_{u}+b(u, v) \partial_{v}
$$

Assim, como por (2.33) e (2.32) resulta que

$$
e_{2}(\mu)=-a(u, v) \cos \theta \cos (c u)
$$

substituindo (2.31) na equação (2.19) segue que

$$
a(u, v)=-\frac{\tan (c u)}{\cos \theta} .
$$


Considerando agora a equação (2.23), e combinando com (2.31), (2.32) e (2.34), temos que

$$
\lambda=-c \tan \theta \tan (c u) .
$$

Além disso, em termos de $u$ e $v$ temos que:

$$
\left[e_{1}, e_{2}\right]=-\frac{\sec ^{2}(c u)}{\cos \theta} \partial_{u}+b_{u}(u, v) \partial_{v} .
$$

Por outro lado, da equação (2.22), resulta

$$
\left[e_{1}, e_{2}\right]=-\frac{c \sec ^{2}(c u)}{\cos \theta} \partial_{u}+c b(u, v) \tan (c u) \partial_{v} .
$$

Igualando as duas equações anteriores e resolvendo a equação diferencial com respeito a $u$ segue que:

$$
b(u, v)=\frac{b_{0}(v)}{\cos (c u)} .
$$

Após uma homotetia na coordenada $v$ de forma que $b_{0}(v)=1$, podemos escrever

$$
b(u, v)=\frac{1}{\cos (c u)} .
$$

Substituindo agora as equações (2.34) e (2.36) em (2.33), temos que a expressão de $e_{2}$ é dada explicitamente por

$$
e_{2}=-\frac{\tan (c u)}{\cos \theta} \partial_{u}+\frac{1}{\cos (c u)} \partial_{v} .
$$

Com isso, a conexão de Levi-Civita de $M$ nas coordenadas $u$ e $v$ pode ser expressa da seguinte forma:

$$
\begin{gathered}
\nabla_{\partial_{u}} \partial_{u}=\frac{c}{\cos \theta}\left(-\frac{\tan (c u)}{\cos \theta} \partial_{u}+\frac{1}{\cos (c u)} \partial_{v}\right) \\
\nabla_{\partial_{u}} \partial_{v}=\nabla_{\partial_{v}} \partial_{u}=c \tan ^{2} \theta \sin (c u)\left(-\frac{\tan (c u)}{\cos \theta} \partial_{u}+\frac{1}{\cos (c u)} \partial_{v}\right) \\
\nabla_{\partial_{v}} \partial_{v}=c \tan ^{2} \theta \sin (c u) \cos (c u) \partial_{u} \\
+\frac{c \tan ^{2} \theta}{\cos \theta} \sin ^{2}(c u)\left(-\frac{\tan (c u)}{\cos \theta} \partial_{u}+\frac{1}{\cos (c u)} \partial_{v}\right) .
\end{gathered}
$$

Substituindo as expressões de $\psi, \mu$ e $\lambda$ na matriz do operador forma, obtemos

$$
A=\left(\begin{array}{cc}
c \tan \theta \cot (c u) & 0 \\
0 & -c \tan \theta \tan (c u)
\end{array}\right) .
$$


Assim as expressões da segunda forma fundamental em termos de $u$ e $v$ são dadas por:

$$
\begin{gathered}
h\left(\partial_{u}, \partial_{u}\right)=c \tan \theta \cot (c u) N, \\
h\left(\partial_{u}, \partial_{v}\right)=h\left(\partial_{v}, \partial_{u}\right)=\frac{c \tan \theta \cos (c u)}{\cos \theta} N, \\
h\left(\partial_{v}, \partial_{v}\right)=c \tan ^{3} \theta \sin (c u) \cos (c u) N .
\end{gathered}
$$

Agora, como a superfície é dada em coordenadas cilíndricas pela imersão isométrica

$$
F: D \subset \mathbb{R}^{2} \longrightarrow \mathbb{R}^{3}
$$

usando a conexão Euclidiana dada por (2.25) temos

$$
\bar{\nabla}_{\partial_{u}} \partial_{u}=\left(r_{u u}-r \phi_{u}^{2}, \phi_{u u}+2 \frac{r_{u}}{r} \phi_{u}, z_{u u}\right) .
$$

Por outro lado, usando a fórmula (1.1), temos

$$
\bar{\nabla}_{\partial_{u}} \partial_{u}=\left(\frac{c \sin ^{2}(c u)+c \sin ^{2} \theta \cos ^{2}(c u)}{\cos \theta \sin (c u)},-\frac{c^{2} \tan \theta \cos (c u)}{\sin ^{2}(c u)}, c \cos \theta \cos (c u)\right) .
$$

Comparando as equações (2.45) e (2.46) obtemos as seguintes equações diferencias:

$$
\begin{gathered}
r_{u u}-r \phi_{u}^{2}=\frac{c \sin (c u)}{\cos \theta}+\frac{c \sin ^{2} \theta \cos ^{2}(c u)}{\cos \theta \sin (c u)}, \\
\phi_{u u}+2 \frac{r_{u}}{r} \phi_{u}=-\frac{c^{2} \tan \theta \cos (c u)}{\sin ^{2}(c u)} \\
z_{u u}=c \cos \theta \cos (c u) .
\end{gathered}
$$

Usando, então, a mesma técnica para as expressões de $\bar{\nabla}_{\partial_{u}} \partial_{v}$ e $\bar{\nabla}_{\partial_{v}} \partial_{v}$ temos:

$$
\begin{aligned}
r_{u v}-r \phi_{u} \phi_{v} & =c \tan ^{2} \theta \\
\phi_{u v}+\frac{1}{r}\left(r_{u} \phi_{v}+r_{v} \phi_{u}\right) & =-\frac{c^{2} \tan \theta \cot (c u)}{\cos \theta}, \\
z_{u v} & =0
\end{aligned}
$$

$\mathrm{e}$

$$
\begin{gathered}
r_{v v}-r \phi_{v}^{2}=\frac{c \tan ^{2} \theta \sin (c u)}{\cos \theta} \\
\phi_{v v}+2 \frac{r_{v}}{r} \phi_{v}=0, \\
z_{v v}=0,
\end{gathered}
$$


respectivamente. Como

$$
r(u, v)=\mu(u)=-\frac{\cos \theta \sin (c u)}{c},
$$

resulta que $r_{v}=0$. Além disso, da escolha de $u$ e $v$, juntamente com (2.26), após uma translação ao longo do eixo $O z$, obtemos a terceira componente da parametrização:

$$
z(u, v)=v-\frac{\cos \theta \cos (c u)}{c} .
$$

Além disso, é simples verificar que

$$
\phi(u, v)=-\frac{c v \tan \theta}{\cos \theta}-\tan \theta \log \left(\tan \frac{c u}{2}\right)
$$

e que as equações (2.47), (2.48) e (2.49) são todas satisfeitas. Assim, quando $\psi \neq 0$, combinando (2.32), (2.50) e (2.51), obtemos a seguinte parametrização em coordenadas cilíndricas:

$$
F(u, v)=\left(-\frac{\cos \theta \sin (c u)}{c},-\frac{c v \tan \theta}{\cos \theta}-\tan \theta \log \left(\tan \frac{c u}{2}, v-\frac{\cos \theta \cos (c u)}{c}\right)\right),
$$

onde $c$ é uma constante real não nula.

Analisemos agora o caso em que $\psi=0$. Seguindo exatamente os mesmo passos feitos no caso geral, obtemos a seguinte expressão

$$
F(u, v)=\left(u \cos \theta, \log \left(c u^{-\tan \theta}\right), v\right), \quad c \in \mathbb{R}-\{0\} .
$$

Essas superfícies são cilindros retos sobre espirais logarítmicas.

Agora estamos aptos a enunciar o teorema principal dessa seção.

Teorema 2.2.2. Seja $M$ uma superfície isometricamente imersa em $\mathbb{R}^{3} \backslash O z$, e considere o campo de Killing $V=-y \partial x+x \partial y$. Então $M$ faz ângulo constante com $V$ se, e somente se, $M$ é uma das seguintes superfícies (a menos de translações verticais ao longo do eixo $O z$ ):

1. um semi-plano com fronteira o eixo $O z$;

2. uma superfície de revolução ao redor do eixo $O z$;

3. um cilindro reto sobre as espirais logarítmicas dadas por (2.53);

4. a superfície de Dini dada em coordenadas cilíndricas por (2.52) (ver Figura 2.8). 


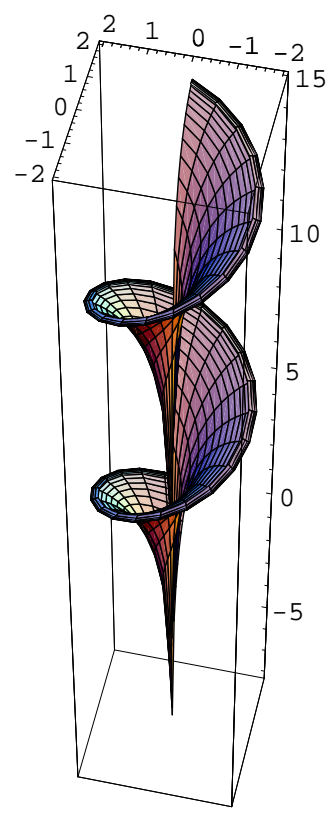

Figura 2.8: A superfície de Dini.

Demonstração. Analisaremos as possibilidades para o ângulo $\theta$ de acordo com as contas feitas anteriormente. Temos que:

- $\operatorname{se} \theta=0$, obtemos semi-planos tendo o eixo $O z$ como fronteira.

- Se $\theta=\pi / 2$, obtemos superfícies de revolução.

- Se $\theta \neq 0, \pi / 2$ e o ângulo $\psi$ obtido anteriormente é 0 , obtemos cilindros retos sobre espirais logarítmicas como em (2.53). Se $\psi \neq 0$ obtemos a superfície de Dini dada em 2.52).

Reciprocamente, se $M$ é uma superfície como no item 1, é óbvio que faz ângulo constante com o campo $V$, que está contido no plano $x y$. Se $M$ é do tipo 2, temos que, localmente, ela pode ser descrita da forma:

$$
F(u, v)=\left(u, v, g\left(\sqrt{u^{2}+v^{2}}\right)\right)
$$


onde $g \in C^{\infty}(\mathbb{R})$. Assim, por meio de contas simples, é possível verificar que ela faz ângulo constante com $V$. Agora se $M$ é do tipo 3, ou 4, temos que $M$ pode ser parametrizada por

$$
\begin{aligned}
F(u, v)= & \left(\left[-\frac{\cos \theta \sin (c u)}{c}\right] \cos \left[-\frac{c v \tan \theta}{\cos \theta}-\tan \theta \ln \left(\tan \left(\frac{c u}{2}\right)\right)\right],\right. \\
& {\left[-\frac{\cos \theta \sin (c u)}{c}\right] \sin \left[-\frac{c v \tan \theta}{\cos \theta}-\tan \theta \ln \left(\tan \left(\frac{c u}{2}\right)\right)\right], } \\
& \left.v-\frac{\cos \theta \cos (c u)}{c}\right)
\end{aligned}
$$

assim, obtemos que

$$
\begin{aligned}
F_{u} \times F_{v}= & \left(\operatorname { c o s } ( c u ) \left[\cos (c u) \cos \left[\left(\ln \left(\tan \left[\frac{c u}{2}\right]\right)+c v \sec \theta\right) \tan \theta\right] \sin \theta\right.\right. \\
& +\cos \theta \sin \left[\left(\ln \left(\tan \left[\frac{c u}{2}\right]\right)+c v \sec \theta\right) \tan \theta\right], \cos (c u)\left[\operatorname { c o s } \theta \operatorname { c o s } \left[\left(\ln \left[\tan \left(\frac{c u}{2}\right)\right]\right.\right.\right. \\
& +c v \sec \theta) \tan \theta]-\cos (c u) \sin \theta \sin \left[\left(\ln \left[\tan \left(\frac{c u}{2}\right)\right]+c v \sec \theta\right) \tan \theta\right], \\
& \left.-\frac{1}{2} \sin (2 c u) \sin \theta\right) .
\end{aligned}
$$

Além disso

$$
\begin{aligned}
V_{\mid M} & =\left(\left[\frac{\cos \theta \sin (c u)}{2}\right] \sin \left[-\frac{c v \tan \theta}{\cos \theta}-\tan \theta \ln \left[\tan \left(\frac{c u}{2}\right)\right]\right],\right. \\
& \left.-\frac{\cos \theta \sin (c u)}{c} \cos \left[-\frac{c v \tan \theta}{\cos \theta}-\tan \theta \ln \left[\tan \left(\frac{c u}{2}\right)\right]\right], 0\right) .
\end{aligned}
$$

Observando que

$$
\left\|F_{u} \times F_{v}\right\|^{2}=\cos ^{2}(c u) \quad \text { e } \quad\|V\|^{2}=\frac{\cos ^{2} \theta \sin ^{2}(c u)}{c^{2}},
$$

segue que

$$
\frac{\langle N, V\rangle}{\|V\|}=\cos \theta
$$





\begin{tabular}{l|c|}
\hline CAPÍtULo \\
\cline { 2 - 2 } \\
3 \\
\hline
\end{tabular}

\section{Superfícies de ângulo constante no grupo de Heisenberg}

Neste capítulo apresentaremos com mais detalhes os resultados do artigo [16] sobre a classificação completa das superfícies de ângulo constante no grupo de Heisenberg tridimensional e, também, alguns resultados parciais sobre a mesma categoria de superfícies em outros espaços homogêneos tridimensionais (com grupo de isometrias de dimensão 4).

Recentemente foram publicados vários trabalhos inerentes ao estudo desta classe de superfícies em variedades tridimensionais (ver, por exemplo, [16, 23, 24, 26, 27, 25]).

No caso do espaço ambiente ser do tipo produto $\mathbb{Q}_{\epsilon}^{2} \times \mathbb{R}$, com

$$
\mathbb{Q}_{\epsilon}^{2}= \begin{cases}\mathbb{R}^{2}, & \epsilon=0 \\ \mathbb{S}^{2}, & \epsilon=1 \\ \mathbb{H}^{2}, & \epsilon=-1\end{cases}
$$


nos artigos [7, 13, 12] foi considerada $\mathbb{R}$ como direção privilegiada (com a qual o vetor normal unitário à superfície faz um ângulo constante) e uma primeira observação que pode-se fazer é que:

- se $\theta=0$, a superfície é uma folha $\mathbb{Q}_{\epsilon}^{2} \times\left\{t_{0}\right\}, t_{0} \in \mathbb{R}$;

- se $\theta=\pi / 2$, a superfície é produto de uma curva em $\mathbb{Q}_{\epsilon}^{2} \operatorname{com} \mathbb{R}$.

Lembramos que os espaços $\mathbb{Q}_{\epsilon}^{2} \times \mathbb{R}$ são exemplos de espaços de Bianchi-Cartan-Vranceanu (definidos na Seção 3.1.1). No trabalho [16] os autores J. Fastenakels, M.I. Munteanu e J. Van der Veken generalizam o conceito de superfícies de ângulo constante (dado em [7, 13, 12]) a um espaço de Bianchi-Cartan-Vranceanu qualquer e mostram que este tipo de superfícies têm curvatura Gaussiana constante (veja Teorema 3.1.7). Além disso, exibem a classificação delas no caso do grupo de Heisenberg (ver Teorema 3.4.1).

\subsection{Espaços homogêneos de dimensão três}

Lembramos que uma variedades Riemanniana é dita homogênea se, para cada par de pontos sobre ela, existe uma isometria que leva um no outro. São exemplos de variedades homogêneas $\mathbb{R}^{3}$, a esfera $\mathbb{S}^{3}$ e o espaço hiperbólico $\mathbb{H}^{3}$. Cartan provou que existem três classes de espaços homogêneos 3-dimensionais simplesmente conexos, dependendo da dimensão do grupo de isometrias da variedade. Essa dimensão pode ser 3,4 ou 6.

Teorema 3.1.1. Seja $(M, g)$ uma variedade Riemanniana conexa, de dimensão $n \geq 2$. O grupo de isometrias I som $(M)$ tem dimensão $r \leq \frac{n(n+1)}{2}$. A igualdade $r=\frac{n(n+1)}{2}$ vale se, e somente se, $(M, g)$ tem curvatura constante.

Segue deste teorema que, dada uma variedade Riemanniana 3-dimensional, seu grupo de isometrias terá dimensão no máximo 6 . Além disso, em virtude do teorema que segue não existem 3 -variedades com grupo de isometrias de dimensão 5.

Teorema 3.1.2 (G. Fubini). Nenhuma variedade Riemanniana $(M, g)$ de dimensão $n \geq 2$ admite um grupo de dimensão $\frac{n(n+1)}{2}-1$, como grupo de isometrias completo ou parcial. 
Além disso, se uma 3-variedade Riemanniana tem grupo de isometria de dimensão 4, então ela é homogênea (ver [22]).

No caso da dimensão ser 6 , a variedade é uma forma espacial. Se a dimensão do grupo de isometrias for 4 , a variedade é isométrica a: $\mathbb{H}_{3}$ (grupo de Heisenberg tridimensional), $\mathbb{S}_{b}^{3}$ (esferas de Berger), $\widetilde{S L}(2, \mathbb{R})$ (revestimento universal do grupo linear especial), ou aos espaços produtos $\mathbb{S}^{2} \times \mathbb{R}$ ou $\mathbb{H}^{2} \times \mathbb{R}$. No caso do grupo de isometrias ser de dimensão três, a variedade possui a geometria do grupo de $\mathrm{Lie} \mathrm{Sol}_{3}$.

\subsubsection{Os espaços de Bianchi-Cartan-Vranceanu}

Feita exceção para $\mathbb{H}^{3}$, no caso em que a dimensão do grupo de isometrias do espaço homogêneo tridimensional é 4 ou 6 , o espaço é localmente isométrico a (uma parte de) $\mathbb{R}^{3}$, munido de uma métrica que depende de dois parâmetros reais. Tal família de métricas Riemannianas aparece primeiramente em 1897 no trabalho [5] do matemático italiano L. Bianchi e, mais tarde, nos artigos [7, 34] de É. Cartan e G. Vranceanu. Por esta razão, estes espaços são conhecidos como variedades de Bianchi-Cartan-Vranceanu (ou BCV-espaços). Temos a seguinte definição:

Definição 3.1.3. Sejam $k$ e $\tau$ números reais, $\operatorname{com} \tau \geq 0$. O espaço de Bianchi-Cartan-Vranceanu $\widetilde{M^{3}}(k, \tau)$ é definido como sendo o conjunto:

$$
\left\{(x, y, z) \in \mathbb{R}^{3} ; 1+\frac{k}{4}\left(x^{2}+y^{2}\right)>0\right\},
$$

equipado com a métrica:

$$
d s^{2}=\frac{d x^{2}+d y^{2}}{\left(1+\frac{k}{4}\left(x^{2}+y^{2}\right)\right)^{2}}+\left[d z+\tau\left(\frac{y d x-x d y}{1+\frac{k}{4}\left(x^{2}+y^{2}\right)}\right)\right]^{2} .
$$

Temos que a variedade $\widetilde{M^{3}}(k, \tau)$ é (localmente) isométrica às seguintes variedades homogêneas tridimensionais (ver [30]):

- se $k=\tau=0$, então $\widetilde{M^{3}}(k, \tau) \cong \mathbb{R}^{3}$;

- se $k=4 \tau^{2} \neq 0$, então $\widetilde{M^{3}}(k, \tau) \cong \mathbb{S}^{3}\left(\frac{k}{4}\right)$;

- se $k>0$ e $\tau=0$, então $\widetilde{M^{3}}(k, \tau) \cong \mathbb{S}^{2}(k) \times \mathbb{R}$; 
- se $k<0$ e $\tau=0$, então $\widetilde{M^{3}}(k, \tau) \cong \mathbb{H}^{2}(k) \times \mathbb{R}$;

- se $k>0$ e $\tau \neq 0$, então $\widetilde{M}^{3}(k, \tau) \cong \mathbb{S}_{b}^{3}$ (esferas de Berger);

- se $k<0$ e $\tau \neq 0$, então $\widetilde{M^{3}}(k, \tau) \cong \widetilde{S L}(2, \mathbb{R})$ (revestimento universal do grupo especial linear);

- se $k=0$ e $\tau \neq 0$, então $\widetilde{M}^{3}(k, \tau) \cong \mathbb{H}_{3}$ (grupo de Heisenberg tridimensional).

Com o intuito de estudar as superfícies de ângulo constante no espaço de Heisenberg e, também, apresentar alguns resultados parciais para a mesma classe de superfícies nos outros BCVespaços com grupo de isometrias 4-dimensional, vamos primeiramente estudar a geometria dos BCV-espaços.

Lema 3.1.4. Os seguintes campos vetoriais formam uma base ortonormal em $\widetilde{M}^{3}(k, \tau)$ :

$$
e_{1}=\left[1+\frac{k}{4}\left(x^{2}+y^{2}\right)\right] \partial_{x}-\tau y \partial_{z}, \quad e_{2}=\left[1+\frac{k}{4}\left(x^{2}+y^{2}\right)\right] \partial_{y}+\tau x \partial_{z}, \quad e_{3}=\partial_{z} .
$$

Além disso, a geometria dos BCV-espaços pode ser descrita em termos dessa base como segue:

1. os campos vetoriais acima satisfazem as relações de comutação:

$$
\begin{aligned}
& {\left[e_{1}, e_{2}\right]=-\frac{k}{2} y e_{1}+\frac{k}{2} x e_{2}+2 \tau e_{3},} \\
& {\left[e_{2}, e_{3}\right]=0} \\
& {\left[e_{3}, e_{1}\right]=0}
\end{aligned}
$$

2. A conexão de Levi-Civita $\bar{\nabla}$ de $\widetilde{M}^{3}(k, \tau)$ é dada por:

$$
\begin{aligned}
& \bar{\nabla}_{e_{1}} e_{1}=\frac{k}{2} y e_{2}, \quad \quad \bar{\nabla}_{e_{1}} e_{2}=-\frac{k}{2} y e_{1}+\tau e_{3}, \quad \bar{\nabla}_{e_{1}} e_{3}=-\tau e_{2}, \\
& \bar{\nabla}_{e_{2}} e_{1}=-\frac{k}{2} x e_{2}-\tau e_{3}, \quad \bar{\nabla}_{e_{2}} e_{2}=\frac{k}{2} x e_{1}, \quad \bar{\nabla}_{e_{2}} e_{3}=\tau e_{1}, \\
& \bar{\nabla}_{e_{3}} e_{1}=-\tau e_{2}, \quad \bar{\nabla}_{e_{3}} e_{2}=\tau e_{1}, \quad \bar{\nabla}_{e_{3}} e_{3}=0 .
\end{aligned}
$$

3. O tensor de curvatura de Riemann-Christoffel $\bar{R}$ de $\widetilde{M^{3}}(k, \tau)$ é dado por:

$$
\begin{aligned}
\bar{R}(X, Y) Z= & \left(k-3 \tau^{2}\right)(\langle Y, Z\rangle X-\langle X, Z\rangle Y) \\
& -\left(k-4 \tau^{2}\right)\left(\left\langle Y, e_{3}\right\rangle\left\langle Z, e_{3}\right\rangle X-\left\langle X, e_{3}\right\rangle\left\langle Z, e_{3}\right\rangle Y\right. \\
& \left.+\left\langle X, e_{3}\right\rangle\langle Y, Z\rangle e_{3}-\left\langle Y, e_{3}\right\rangle\langle X, Z\rangle e_{3}\right),
\end{aligned}
$$

para todo $X, Y, Z \in \chi\left(\widetilde{M^{3}}(k, \tau)\right)$. 
Demonstração. Observando que

$$
e_{1}=\left(1+\frac{k}{4}\left(x^{2}+y^{2}\right), 0,-\tau y\right), \quad e_{2}=\left(0,1+\frac{k}{4}\left(x^{2}+y^{2}\right),-\tau x\right), \quad e_{3}=(0,0,1),
$$

e escrevendo

$$
f=1+\frac{k}{4}\left(x^{2}+y^{2}\right)
$$

temos

$$
\begin{aligned}
d s^{2} & =\frac{d x^{2}}{f^{2}}+\frac{d y^{2}}{f^{2}}+\left(d z+\tau \frac{(y d x-x d y)}{f}\right)^{2} \\
& =\left(\frac{1+\tau^{2} y^{2}}{f^{2}}\right) d x^{2}+\left(\frac{1+\tau^{2} x^{2}}{f^{2}}\right) d y^{2}+d z^{2} \\
& +\frac{2 \tau y}{f} d x d z-\frac{2 \tau x}{f} d y d z-\frac{2 \tau^{2} x y}{f^{2}} d x d y,
\end{aligned}
$$

ou seja, a matriz associada à métrica $d s^{2}$ é dada por

$$
\left(\begin{array}{ccc}
\frac{1+\tau^{2} y^{2}}{f^{2}} & -\frac{\tau^{2} x y}{f^{2}} & \frac{\tau y}{f} \\
-\frac{\tau^{2} x y}{f^{2}} & \frac{1+\tau^{2} x^{2}}{f^{2}} & -\frac{\tau x}{f} \\
\frac{\tau y}{f} & -\frac{\tau x}{f} & 1
\end{array}\right)
$$

1. Temos

$$
\begin{aligned}
{\left[e_{1}, e_{2}\right] } & =\left[f \partial_{x}-\tau y \partial_{z}, f \partial_{y}+\tau x \partial_{z}\right] \\
& =f \partial_{x}(f) \partial_{y}-f \partial_{y}(f) \partial_{x}+\tau f \partial_{z}-\tau x \partial_{x}(f) \partial_{x}+\tau f \partial_{z} \\
& =-y \frac{k}{2}\left(f \partial_{x}-\tau y \partial_{z}\right)+\frac{x k}{2}\left(f \partial_{y}+\tau x \partial_{z}\right)+2 \tau \partial_{z} \\
& =-y \frac{k}{2} e_{1}+\frac{x k}{2} e_{2}+2 \tau e_{3}, \\
{\left[e_{2}, e_{3}\right] } & =\left[f \partial_{y}+\tau x \partial_{z}, \partial_{z}\right]=0, \\
{\left[e_{3}, e_{1}\right] } & =\left[\partial_{z}, f \partial_{x}-\tau y \partial_{z}\right]=0 .
\end{aligned}
$$

2. Pela fórmula de Koszul, resulta que

$$
\left\langle\bar{\nabla}_{e_{1}} e_{1}, e_{1}\right\rangle=0, \quad\left\langle\bar{\nabla}_{e_{1}} e_{1}, e_{3}\right\rangle=0
$$




$$
\begin{aligned}
\left\langle\bar{\nabla}_{e_{1}} e_{1}, e_{2}\right\rangle & =-\frac{1}{2}\left(\left\langle\left[e_{1}, e_{1}\right], e_{2}\right\rangle+\left\langle\left[e_{1}, e_{2}\right], e_{1}\right\rangle+\left\langle\left[e_{1}, e_{2}\right], e_{1}\right\rangle\right) \\
& =\frac{k y}{2}
\end{aligned}
$$

Logo

$$
\bar{\nabla}_{e_{1}} e_{1}=\frac{k y}{2} e_{2}
$$

De forma análoga, obtemos as outras expressões para a conexão.

3. Dado um campo de vetores $X$ em $\widetilde{M}^{3}(k, \tau)$, podemos verificar que

$$
\bar{\nabla}_{X} e_{3}=\tau X \times e_{3}
$$

onde $\times$ representa o produto vetorial em $\widetilde{M}^{3}(k, \tau)$. Pondo

$$
\langle\bar{R}(X \times Y), Z \times W\rangle=\langle\bar{R}(X, Y) Z, W\rangle,
$$

e usando que

$$
\langle\bar{R}(X, Y) Z, W\rangle=\bar{\nabla}_{X} \bar{\nabla}_{Y} Z-\bar{\nabla}_{Y} \bar{\nabla}_{X} Z-\bar{\nabla}_{[X, Y]} Z,
$$

obtemos que a matriz de $\bar{R}$ na base $\left\{e_{2} \times e_{3}, e_{3} \times e_{1}, e_{1} \times e_{2}\right\}$ é dada por

$$
\bar{R}=\left(\begin{array}{ccc}
-\tau^{2} & 0 & 0 \\
0 & -\tau^{2} & 0 \\
0 & 0 & 3 \tau^{2}-k
\end{array}\right)
$$

Decompondo os campos $X, Y, Z, W$ na forma:

$$
X=\bar{X}+x e_{3}, \quad Y=\bar{Y}+y e_{3}, \quad Z=\bar{Z}+z e_{3} \quad \text { e } \quad W=\bar{W}+w e_{3},
$$

onde $\bar{X}, \bar{Y}, \bar{Z}, \bar{W}$ são as componentes horizontais obtemos:

$$
\begin{aligned}
\langle\bar{R}(X, Y) Z, W\rangle= & \langle\bar{R}(\bar{X}, \bar{Y}) \bar{Z}, \bar{W}\rangle+z\left\langle\bar{R}(\bar{X}, \bar{Y}) e_{3}, \bar{W}\right\rangle \\
& +y\left\langle\bar{R}\left(\bar{X}, e_{3}\right) \bar{Z}, \bar{W}\right\rangle+y z\left\langle\bar{R}\left(\bar{X}, e_{3}\right) e_{3}, \bar{W}\right\rangle \\
& +x\left\langle\bar{R}\left(e_{3}, \bar{Y}\right) \bar{Z}, \bar{W}\right\rangle+x z\left\langle\bar{R}\left(e_{3}, \bar{Y}\right) e_{3}, \bar{W}\right\rangle \\
& +w\left\langle\bar{R}(\bar{X}, \bar{Y}) \bar{Z}, e_{3}\right\rangle+w z\left\langle\bar{R}(\bar{X}, \bar{Y}) e_{3}, e_{3}\right\rangle \\
& +y w\left\langle\bar{R}\left(\bar{X}, e_{3}\right) \bar{Z}, e_{3}\right\rangle+y z w\left\langle\bar{R}\left(\bar{X}, e_{3}\right) e_{3}, e_{3}\right\rangle \\
& +x w\left\langle\bar{R}\left(e_{3}, \bar{Y}\right) \bar{Z}, e_{3}\right\rangle+x z w\left\langle\bar{R}\left(e_{3}, \bar{Y}\right) e_{3}, e_{3}\right\rangle .
\end{aligned}
$$


Observemos agora que, como a matriz de $\bar{R}$ é diagonal, os termos em que $e_{3}$ aparece somente uma vez são nulos, uma vez que $\bar{X}, \bar{Y}, \bar{Z}$ e $\bar{W}$ dependem somente de $e_{1}, e_{2}$, assim

$$
\begin{aligned}
\langle\bar{R}(X, Y) Z, W\rangle= & \langle\bar{R}(\bar{X}, \bar{Y}) \bar{Z}, \bar{W}\rangle+z y\left\langle\bar{R}\left(\bar{X}, e_{3}\right) e_{3}, \bar{W}\right\rangle \\
& +x z\left\langle\bar{R}\left(e_{3}, \bar{Y}\right) e_{3}, \bar{W}\right\rangle+w y\left\langle\bar{R}\left(\bar{X}, e_{3}\right) \bar{Z}, e_{3}\right\rangle \\
& +w x\left\langle\bar{R}\left(e_{3}, \bar{Y}\right) \bar{Z}, e_{3}\right\rangle .
\end{aligned}
$$

Escrevendo, então

$$
X=\sum_{i=1}^{3} a_{i} e_{i}, \quad Y=\sum_{i=1}^{3} b_{i} e_{i} \quad \text { e } \quad Z=\sum_{i=1}^{3} z_{i} e_{i}
$$

obtemos que:

$$
\begin{aligned}
\langle\bar{R}(\bar{X}, \bar{Y}) \bar{Z}, \bar{W}\rangle & =\sum_{i, j, k, s=1}^{2} a_{i} b_{j} z_{k} w_{s}\left\langle\bar{R}\left(e_{i}, e_{j}\right) e_{k}, e_{s}\right\rangle \\
& =\left(a_{1} z_{1} b_{2} w_{2}+a_{2} z_{2} b_{1} w_{1}-\left(a_{2} w_{2} b_{1} z_{1}+a_{1} w_{1} b_{2} w_{2}\right)\right)\left\langle\bar{R}\left(e_{1}, e_{2}\right) e_{1}, e_{2}\right\rangle \\
& =\left(k-3 \tau^{2}\right)(\langle\bar{X}, \bar{W}\rangle\langle\bar{Y}, \bar{Z}\rangle-\langle\bar{X}, \bar{Z}\rangle\langle\bar{Y}, \bar{W}\rangle)
\end{aligned}
$$

$\mathrm{e}$

$$
\begin{aligned}
R\left(\bar{X}, e_{3}\right) e_{3} & =\bar{\nabla}_{\bar{X}} \bar{\nabla}_{e_{3}} e_{3}-\bar{\nabla}_{e_{3}} \bar{\nabla}_{\bar{X}} e_{3}-\bar{\nabla}_{\left[\bar{X}, e_{3}\right]} e_{3} \\
& =-\bar{\nabla}_{e_{3}}\left(-\tau a_{1} e_{2}+\tau a_{2} e_{1}\right)-\tau e_{3}\left(a_{1}\right) e_{2}-\tau e_{3}\left(a_{2}\right) e_{1} \\
& =\tau^{2} \bar{X}
\end{aligned}
$$

para todo campo $X \in \chi\left(\widetilde{M^{3}}(k, \tau)\right)$. Segue que

$$
\begin{aligned}
\langle\bar{R}(X, Y) Z, W\rangle & =\left(k-3 \tau^{2}\right)(\langle\bar{X}, \bar{W}\rangle\langle\bar{Y}, \bar{Z}\rangle-\langle\bar{X}, \bar{Z}\rangle\langle\bar{Y}, \bar{W}\rangle) \\
& =-\tau^{2}(-z y\langle\bar{X}, \bar{W}\rangle+x z\langle\bar{Y}, \bar{W}\rangle+w y\langle\bar{X}, \bar{Z}\rangle-w x\langle\bar{Y}, \bar{Z}\rangle) .
\end{aligned}
$$

Observando que

$$
\begin{aligned}
\langle\bar{X}, \bar{Z}\rangle\langle\bar{Y}, \bar{W}\rangle & =\langle X, Z\rangle\langle Y, W\rangle-y w\langle\bar{X}, \bar{Z}\rangle-x z\langle\bar{Y}, \bar{W}\rangle-x z y w \\
\langle\bar{X}, \bar{W}\rangle\langle\bar{Y}, \bar{Z}\rangle & =\langle X, W\rangle\langle Y, Z\rangle-x w\langle\bar{Y}, \bar{Z}\rangle-y z\langle\bar{X}, \bar{W}\rangle-x z y w
\end{aligned}
$$

e substituindo essas equações em (3.3), tendo em conta que

$$
\begin{aligned}
x z\langle\bar{Y}, \bar{W}\rangle & =x z\langle Y, W\rangle-x y z w, \\
y w\langle\bar{Z}, \bar{X}\rangle & =w y\langle Z, X\rangle-x y z w, \\
-z y\langle\bar{X}, \bar{W}\rangle & =-z y\langle X, W\rangle+x y z w, \\
-x w\langle\bar{Z}, \bar{Y}\rangle & =-x w\langle Z, Y\rangle+x y z w,
\end{aligned}
$$


e

$$
x=\left\langle X, e_{3}\right\rangle, \quad y=\left\langle Y, e_{3}\right\rangle, \quad z=\left\langle Z, e_{3}\right\rangle, \quad w=\left\langle W, e_{3}\right\rangle,
$$

resulta

$$
\begin{aligned}
\langle\bar{R}(X, Y) Z, W\rangle= & -3 \tau^{2}(\langle X, W\rangle\langle Y, Z\rangle-\langle X, Z\rangle\langle Y, W\rangle) \\
& -\left(k-4 \tau^{2}\right)\left(\langle X, W\rangle\left\langle Z, e_{3}\right\rangle\left\langle Y, e_{3}\right\rangle+\langle Z, Y\rangle\left\langle X, e_{3}\right\rangle\left\langle W, e_{3}\right\rangle\right. \\
& \left.-\langle Y, W\rangle\left\langle X, e_{3}\right\rangle\left\langle Z, e_{3}\right\rangle-\langle Z, X\rangle\left\langle W, e_{3}\right\rangle\left\langle Z, e_{3}\right\rangle\right) .
\end{aligned}
$$

Isto completa a demonstração.

Definição 3.1.5. Seja $\widetilde{M}^{3}(k)$ a superfície Riemanniana, com curvatura Gaussiana constante igual a $k$, dada por:

$$
\widetilde{M}^{2}(k)=\left(\left\{(x, y) \in \mathbb{R}^{2} \mid 1+\frac{k}{4}\left(x^{2}+y^{2}\right)>0\right\}, \frac{d x^{2}+d y^{2}}{\left(1+\frac{k}{4}\left(x^{2}+y^{2}\right)\right)^{2}}\right) .
$$

Então a aplicação

$$
\begin{aligned}
\pi: \widetilde{M^{3}}(k, \tau) & \longrightarrow \widetilde{M}^{2}(k) \\
(x, y, z) & \longmapsto(x, y)
\end{aligned}
$$

é uma submersão Riemanniana chamada fibração de Hopf. A imagem inversa de uma curva em $\widetilde{M}^{2}(k)$ pela aplicação $\pi$ é chamada cilindro de Hopf e a folha da fibração de Hopf é uma superfície que é ortogonal às fibras de $\pi$ em todo ponto.

Observação 3.1.6. No caso especial em que $k=4 \tau^{2} \neq 0$, a aplicação $\pi$ coincide localmente com a fibração de Hopf clássica $\pi: \mathbb{S}^{3}(k / 4) \rightarrow \mathbb{S}^{2}(k)$. Do Teorema de Frobenius e do item (1) do Lema 3.1.4 as folhas de $\pi$ existem se, e somente se, $\tau=0$. Elas nada mais são do que abertos de superfícies do tipo $\mathbb{R}^{2} \times\left\{t_{0}\right\}, \mathbb{S}^{2}(k) \times\left\{t_{0}\right\}$ ou $\mathbb{H}^{2}(k) \times\left\{t_{0}\right\}$.

\subsubsection{Imersões isométricas em BCV-espaços}

Sejam $M^{n}$ e $N^{n+1}$ variedades Riemannianas, com conexão de Levi-Civita $\nabla$ e $\bar{\nabla}$ e cujo tensor curvatura é $R$ e $\bar{R}$ (respectivamente), então as equações de Gauss e Codazzi dadas por:

$$
\begin{gathered}
\langle R(X, Y) Z, W\rangle-\langle\bar{R}(X, Y) Z, W\rangle=\langle A(X), Z\rangle\langle A(Y), W\rangle-\langle A(Y), Z\rangle\langle A(X), W\rangle, \\
\nabla_{X} A(Y)-\nabla_{Y} A(X)-A[X, Y]=-\bar{R}(X, Y) N, \quad X, Y, Z, W \in \chi(M),
\end{gathered}
$$


são condições necessárias para que $M$ possa ser imersa isometricamente em $N$. Além disso, no caso em que $N$ é uma forma espacial (i.e. a esfera $\mathbb{S}^{n+1}$, o espaço Euclidiano $\mathbb{R}^{n+1}$ e o espaço hiperbólico $\mathbb{H}^{n+1}$ ), as equações de Gauss e de Codazzi representam, também, condições suficientes para que $M$ possa ser imersa isometricamente em $N$, com $A$ como operador de forma. Neste caso estas equações envolvem somente a métrica e o operador de forma de $M$.

Em [11], foi considerado o problema de determinar quando uma superfície $M^{2}$ pode ser imersa isometricamente em uma variedade homogênea tridimensional $N^{3}$ cujo grupo de isometrias tem dimensão quatro. Como vimos, tais variedades são fibrações Riemannianas sobre uma forma espacial bidimensional, com fibras geodésicas e existe uma família a um parâmetro de translações ao longo das fibras, com gerador o campo de Killing unitário $e_{3}$.

Teorema 3.1.7. [1] Seja $F: M^{2} \longrightarrow \widetilde{M}^{3}(k, \tau)$ uma imersão isométrica de uma superfície orientada $M^{2}$ em um BCV-espaço. Seja $N$ o campo unitário de vetores normais a $M$, A o operador forma associado,

$$
\theta=\angle\left(N, e_{3}\right) \quad \text { e } \quad T=e_{3}+\cos \theta N
$$

Então, se $X, Y \in \chi(M)$, temos:

1. $\nabla_{X} A(Y)-\nabla_{Y} A(X)-A[X, Y]=\left(k-4 \tau^{2}\right) \cos \theta(\langle Y, T\rangle X-\langle X, T\rangle Y)$;

2. $K=\operatorname{det} A+\tau^{2}+\left(k-4 \tau^{2}\right) \cos ^{2} \theta$;

3. $X[\cos \theta]=-\langle A(X)-\tau J X, T\rangle$;

4. $\nabla_{X} T=\cos \theta(A(X)-\tau J X)$,

onde $K$ representa a curvatura gaussiana de $M$.

Demonstração. 1. Da equação de Codazzi e do item (3) do Lema 3.1.4 temos que

$$
\begin{aligned}
\nabla_{X} A(Y)-\nabla_{Y} A(X)-A[X, Y] & =\left(k-4 \tau^{2}\right) \cos \theta\left(\left\langle Y, e_{3}\right\rangle X-\left\langle X, e_{3}\right\rangle Y\right) \\
& =\left(k-4 \tau^{2}\right) \cos \theta(\langle Y, T\rangle X-\langle X, T\rangle Y)
\end{aligned}
$$

2. Segue das equações de Gauss e de Codazzi. 
3. e 4. Definindo $J X=N \times X$, temos

$$
\begin{aligned}
\bar{\nabla}_{X} e_{3} & =\bar{\nabla}_{X}(T+\cos \theta N)=\bar{\nabla}_{X} T+\nabla_{X}(\cos \theta N) \\
& =\nabla_{X} T+h(X, T)+X(\cos \theta) N+\cos \theta \nabla_{X} N
\end{aligned}
$$

Como

$$
h(X, T)=\langle T, A(X)\rangle N,
$$

resulta que

$$
\bar{\nabla}_{X} e_{3}=\nabla_{X} T+\langle h(X, T), T\rangle N+X(\cos \theta) N-\cos \theta A(X) .
$$

Por outro lado, pela equação (3.1) segue que

$$
\begin{aligned}
\bar{\nabla}_{X} e_{3} & =\tau X \times(T+\cos \theta N)=\tau(X \times T)+\tau \cos \theta(X \times N) \\
& =\tau(X \times T)-\tau \cos \theta J X .
\end{aligned}
$$

Notemos agora que, se $\varphi$ é o ângulo entre $X$ e $T$, então o ângulo entre $J X$ e $T$ é $(\varphi+\pi / 2)$. Além disso, como $X$ e $T$ estão no mesmo plano, temos que $X \times T$ é paralelo a $N$, assim

$$
X \times T=\langle J X, T\rangle N
$$

$\log 0$

$$
\bar{\nabla}_{X} e_{3}=\tau\langle J X, T\rangle N-\tau \cos \theta J X .
$$

Combinando (3.4) e (3.5) obtemos

$$
\nabla_{X} T=\cos \theta(A(X)-\tau J X)
$$

$\mathrm{e}$

$$
X[\cos \theta]=-\langle A(X)-\tau J X, T\rangle .
$$

As equações do Teorema 3.1.7] são ditas equações de compatibilidade para $\widetilde{M}^{3}(k, \tau)$ e em [11] também é provado que as mesmas são condições necessárias e suficientes para que uma superfície Riemanniana seja imersa isometricamente numa variedade homogênea tridimensional cujo grupo de isometrias seja de dimensão quatro. 


\subsubsection{Superfície de ângulo constante nos BCV-espaços}

O teorema de existência e unicidade provado por Benôit (ver [11]) mostra como a função ângulo $\cos \theta$ é um dos invariantes fundamentais para uma superfície em um BCV-espaço. Logo, é um problema natural estudar as superfícies para as quais esta função é constante. Desta forma, em [16] J.Fastenakels e M.I. Munteanu, generalizam o conceito de superfícies de ângulo constante dado em $\mathbb{Q}_{\epsilon}^{2} \times \mathbb{R}$ a um BCV-espaço qualquer da seguinte forma:

Definição 3.1.8. Dizemos que uma superfície orientada $M^{2}$ no $\mathrm{BCV}$-espaço $\widetilde{M^{3}}(k, \tau)$ é uma $s u$ perfície de ângulo constante se a função $\cos \theta$ é constante, ou seja, o ângulo entre a normal unitária $N$ e a direção $e_{3}$, tangente às fibras da fibração de Hopf, é o mesmo em todos os pontos.

Lema 3.1.9. Seja $M$ uma superfície de ângulo constante em um $B C V$-espaço $\widetilde{M}^{3}(k, \tau)$. Então valem as seguintes afirmações:

1. o operador de forma com respeito a base $\{T, J T\}$ é dado por:

$$
A=\left(\begin{array}{cc}
0 & -\tau \\
\tau & \lambda
\end{array}\right),
$$

onde $\lambda \in C^{\infty}(M)$.

2. A conexão de Levi-Civita da superfície é determinada por:

$$
\begin{array}{ll}
\nabla_{T} T=-2 \tau \cos \theta J T, & \nabla_{J T} T=\lambda \cos \theta J T, \\
\nabla_{T} J T=2 \tau \cos \theta T, & \nabla_{J T} J T=-\lambda \cos \theta T .
\end{array}
$$

3. A curvatura Gaussiana da superfície é a constante dada por:

$$
K=\left(k-4 \tau^{2}\right) \cos ^{2} \theta
$$

4. A função $\lambda$ satisfaz a seguinte equação diferencial:

$$
T[\lambda]+\lambda^{2} \cos \theta+k \cos \theta \sin ^{2} \theta+4 \tau^{2} \cos ^{3} \theta=0 .
$$

Demonstração. 1. Como o ângulo $\theta$ é constante, dado um campo $X \in \chi\left(\widetilde{M}^{3}(k, \tau)\right)$, temos que

$$
X(\cos \theta)=0 .
$$


Sendo assim do item 3, do Teorema 3.1.7 resulta que

$$
\langle A(X), T\rangle=\langle\tau J X, T\rangle
$$

e, portanto,

$$
\langle A(T), T\rangle=\tau\langle J T, T\rangle=0
$$

$\mathrm{e}$

$$
\langle A(T), J T\rangle=\tau\langle J(J T), T\rangle=-\tau\langle T, T\rangle .
$$

Logo

$$
A(T)=-\tau J T
$$

De forma análoga, obtemos que

$$
A(J T)=-\tau T+\lambda J T
$$

onde $\lambda=\langle A(J T), J T\rangle$. Portanto a matriz de $A$ na base $\{T, J T\}$ é dada como no item 1 .

2. Do item 4, do Teorema 3.1.7 segue que

$$
\nabla_{T} T=\cos \theta(A(T)-\tau J T)=-2 \tau \cos \theta J T .
$$

Além disso, temos

$$
\left\langle\nabla_{T} J T, T\right\rangle=-\left\langle\nabla_{T} T, J T\right\rangle=2 \tau \cos \theta\|J T\|^{2} J T .
$$

Como $\|T\|^{2}=\|J T\|^{2}=\sin ^{2} \theta$, resulta que

$$
\left\langle\nabla_{T} J T, J T\right\rangle=0,
$$

então

$$
\nabla_{T} J T=2 \cos \theta J T
$$

Novamente do Teorema 3.1.7, item 4, temos

$$
\nabla_{J T} T=\cos \theta(A(J T)-\tau J(J T))=\lambda \cos \theta J T .
$$

Por fim, como

$$
\left\langle\nabla_{J T} J T, J T\right\rangle=0
$$


e

$$
\left\langle\nabla_{J T} J T, T\right\rangle=-\left\langle\nabla_{J T} T, J T\right\rangle=-\lambda \cos \theta\|J T\|^{2} J T
$$

segue que

$$
\nabla_{J T} J T=-\lambda \cos \theta J T
$$

3. Do item 11, temos que $\operatorname{det} A=-\tau^{2}$. Assim, do item 2, do Teorema 3.1.7, a curvatura Gaussiana da superfície é dada por

$$
K=\operatorname{det} A+\tau^{2}+\left(k-4 \tau^{2}\right) \cos ^{2} \theta=\left(k-4 \tau^{2}\right) \cos ^{2} \theta .
$$

4. Sabemos que

$$
K(T, J T)=\frac{\langle R(T, J T) J T, T\rangle}{\|T\|^{2}\|J T\|^{2}}=\left(k-4 \tau^{2}\right) \cos ^{2} \theta
$$

Como

$$
R(T, J T) J T=-\cos \theta T[\lambda] T-4 \tau^{2} \cos ^{2} \theta T-\lambda^{2} \cos ^{2} \theta T,
$$

segue que

$$
\begin{aligned}
0 & =\cos \theta T[\lambda]+4 \tau^{2} \cos ^{2} \theta+\lambda^{2} \cos ^{2} \theta+\left(k-4 \tau^{2}\right) \cos ^{4} \theta \\
& =\cos \theta T[\lambda]+4 \tau^{2} \cos ^{4} \theta+k \cos ^{2} \theta \sin ^{2} \theta+\lambda^{2} \cos ^{2} \theta
\end{aligned}
$$

Dado $\cos \theta \neq 0$, obtemos

$$
T[\lambda]+4 \tau^{2} \cos ^{2} \theta+k \cos \theta \sin ^{2} \theta+\lambda^{2} \cos \theta=0 .
$$

Em nosso estudo estamos considerando o caso das superfícies de ângulo constante nos BCVespaços com $k, \tau \neq 0$. Observe que o caso $\theta=0$ não pode ocorrer pois a distribuição gerada por $e_{1}$ e $e_{2}$ não é integrável. Se $\theta=\pi / 2$, temos que o campo $e_{3}$ será tangente à superfície em todo ponto, assim ela conterá as curvas integrais deste campo, as quais se projetam em um ponto. Portanto a projeção da superfície através da aplicação de Hopf será uma curva, ou seja, a superfície será um cilindro de Hopf.

Para obtermos uma classificação local explícita das superfícies de ângulo constante $\theta \in(0, \pi / 2)$ em um BCV-espaço, com $k, \tau \neq 0$, escolhemos primeiramente um sistema de coordenadas locais $(u, v)$ de forma que:

$$
T=\partial_{u} \quad \text { e } \quad \partial_{v}=a T+b J T
$$


onde $a, b \in C^{\infty}(M)$. Teremos, então, que a equação (3.6) poderá ser reescrita como

$$
\lambda_{u}+4 \tau^{2} \cos ^{3} \theta+k \cos \theta \sin ^{2} \theta+\lambda^{2} \cos \theta=0 .
$$

A solução dessa equação depende do sinal da constante $\left(k \sin ^{2} \theta+4 \tau^{2} \cos ^{2} \theta\right)$. Supondo que este seja positivo e denotando essa constante por $r^{2}$, temos

$$
\lambda(u, v)=r \tan (\varphi(v)-r \cos \theta u)
$$

para alguma função real $\varphi(v)$. Temos ainda que, como $\left[\partial_{u}, \partial_{v}\right]=0$, então

$$
0=[T, a T+b J T]=\partial_{u} a T+2 b \tau \cos \theta T+\partial_{u} b J T-b \lambda \cos \theta J T
$$

Obtemos então o sistema de equações:

$$
\left\{\begin{array}{l}
a_{u}=-2 \tau b \cos \theta \\
b_{u}=b \lambda \cos \theta
\end{array}\right.
$$

Da expressão de $\lambda$ segue que

$$
b_{u}=b r \tan (\varphi(v)-r \cos \theta u) \cos \theta
$$

ou seja

$$
b(u, v)=\cos (\varphi(v)-r \cos \theta u) .
$$

Substituindo esta expressão no sistema (3.7) temos

$$
a_{u}=-2 \tau \cos (\varphi(v)-r \cos \theta u) \cos \theta
$$

$\log 0$

$$
a(u, v)=\frac{2 \tau}{r} \sin (\varphi(v)-r \cos \theta u) .
$$

Como $N$ é unitário e a superfície é de ângulo constante existe uma função diferenciável $\phi$, localmente definida em $M$, tal que

$$
N=\sin \theta \cos \phi e_{1}+\sin \theta \sin \phi e_{2}+\cos \theta e_{3} .
$$

Com isso, obtemos

$$
\begin{aligned}
T & =e_{3}-\cos \theta N=e_{3}-\sin \theta \cos \theta \cos \phi e_{1}-\sin \theta \cos \theta \sin \phi e_{2}-\cos ^{2} \theta e_{3} \\
& =-\sin \theta\left(\cos \theta \cos \phi e_{1}+\cos \theta \sin \phi e_{2}-\sin \theta e_{3}\right)
\end{aligned}
$$


$\mathrm{e}$

$$
J T=N \times T=\sin \theta\left(\sin \phi e_{1}-\cos \phi e_{2}\right) .
$$

Temos ainda, pela fórmula de Weingarten, juntamente com o Lema 3.1.4 (item 2) e as equações acima, que

$$
\begin{aligned}
A(T) & =-\bar{\nabla}_{T} N=\left(T[\phi] J T+\sin \theta \cos \theta \frac{k}{2}(\sin \phi x-\cos \phi y)\right. \\
& \left.-\tau \sin ^{2} \theta+\tau \cos ^{2} \theta\right) J T
\end{aligned}
$$

$\mathrm{e}$

$$
A(J T)=-\bar{\nabla}_{J T} N=\left(J T[\phi] J T+\sin \theta \frac{k}{2}(\sin \phi y+\cos \phi x)\right) J T-\tau T .
$$

Comparando com o item 1 do Lema 3.1.9, obtemos

$$
\left\{\begin{aligned}
T[\phi] & =-2 \tau \cos ^{2} \theta \\
J T[\phi] & =\lambda-\sin \theta \frac{k}{2}(\sin \phi y+\cos \phi x) .
\end{aligned}\right.
$$

Observe que a condição de integrabilidade para esse sistema de equações diferenciais é exatamente a equação (3.6).

Seja agora

$$
\begin{aligned}
F: U \subset \mathbb{R}^{2} \longrightarrow M \subset \widetilde{M}^{3}(k, \tau) \\
(u, v) \longmapsto\left(F_{1}(u, v), F_{2}(u, v), F_{3}(u, v)\right)
\end{aligned}
$$

uma parametrização de $M$. Observando que, no ponto $\left(F_{1}(u, v), F_{2}(u, v), F_{3}(u, v)\right)$ temos

$$
e_{1}=\left(1+\frac{k}{4}\left(F_{1}^{2}+F_{2}^{2}\right), 0,-\tau F_{2}\right), \quad e_{2}=\left(0,1+\frac{k}{4}\left(F_{1}^{2}+F_{2}^{2}\right), \tau F_{1}\right), \quad e_{3}=(0,0,1)
$$

e que

$$
\phi_{v}=a(u, v) T[\phi]+b(u, v)\left[\lambda-\frac{k}{2} \sin \theta(\sin \phi y-\cos \phi x)\right]
$$


para encontrar uma parametrização para $M$ precisamos resolver o sistema:

$$
\begin{aligned}
\phi_{u} & =-\frac{k}{2} \sin \theta \cos \theta\left(F_{1} \sin \phi-F_{2} \cos \phi\right)-2 \tau \cos ^{2} \theta \\
\phi_{v} & =a(u, v) \phi_{u}+b(u, v)\left(\lambda(u, v)-\frac{k}{2} \sin \theta\left(F_{1} \cos \phi+F_{2} \sin \phi\right)\right) \\
\left(F_{1}\right)_{u} & =-\sin \theta \cos \theta \cos \phi\left(1+\frac{k}{4}\left(F_{1}^{2}+F_{2}^{2}\right)\right) \\
\left(F_{1}\right)_{v} & =a(u, v)\left(F_{1}\right)_{u}+b(u, v) \sin \theta \sin \phi\left(1+\frac{k}{4}\left(F_{1}^{2}+F_{2}^{2}\right)\right) \\
\left(F_{2}\right)_{u} & =-\sin \theta \cos \theta \sin \phi\left(1+\frac{k}{4}\left(F_{1}^{2}+F_{2}^{2}\right)\right) \\
\left(F_{2}\right)_{v} & =a(u, v)\left(F_{2}\right)_{u}-b(u, v) \sin \theta \cos \phi\left(1+\frac{k}{4}\left(F_{1}^{2}+F_{2}^{2}\right)\right) \\
\left(F_{3}\right)_{u} & =-\sin \theta\left(-\tau F_{2} \cos \theta \cos \phi+\tau F_{1} \cos \theta \sin \phi-\sin \theta\right) \\
\left(F_{3}\right)_{v} & =a(u, v)\left(F_{3}\right)_{u}-b(u, v) \tau \sin \theta\left(F_{2} \sin \phi+F_{1} \cos \phi\right)
\end{aligned}
$$

De 3.9, 3.11) e 3.13), obtemos:

$$
\begin{aligned}
F_{1} & =\frac{\sin 2 \theta}{2 D(v)} \sin \phi+L(v) \cos (\rho(v)), \\
F_{2} & =-\frac{\sin 2 \theta}{2 D(v)} \cos \phi+L(v) \sin (\rho(v)), \\
\phi & =\rho(v)+2 \arctan \left(\frac{-A+\sqrt{B^{2}-A^{2}} \tan \left(-\frac{1}{2} \sqrt{B^{2}-A^{2}} u+C(v)\right)}{B}\right)
\end{aligned}
$$

onde $D(v), L(v), \rho(v)$ e $C(v)$ são constantes de integração e

$$
A(v)=\frac{k}{4} \sin 2 \theta L(v), \quad B(v)=D(v)+\frac{k}{4}\left(\frac{\sin ^{2} 2 \theta}{4 D(v)}+D(v) L^{2}(v)\right) .
$$

Observe que $B^{2}-A^{2}=r^{2} \cos ^{2} \theta$ é uma constante positiva.

\subsection{O grupo de Heisenberg}

Começaremos dando a seguinte

Definição 3.2.1. Seja $(V, \omega)$ um espaço vetorial simplético de dimensão $2 n$. O grupo de Heisenberg associado a ele é o conjunto $V \times \mathbb{R}$ munido da operação:

$$
\left(v_{1}, t_{1}\right) *\left(v_{2}, t_{2}\right)=\left(v_{1}+v_{2}, t_{1}+t_{2}+\frac{1}{2} \omega\left(v_{1}, v_{2}\right)\right) .
$$


Nesta seção nos restringiremos ao grupo de Heisenberg tridimensional vindo de $\mathbb{R}^{2}$ com a forma simplética canônica:

$$
\omega((x, y),(\bar{x}, \bar{y}))=(x \bar{y}-\bar{x} y)
$$

isto é, estaremos considerando $\mathbb{R}^{3}$ com a operação de grupo dada por:

$$
(x, y, z) *(\bar{x}, \bar{y}, \bar{z})=\left(x+\bar{x}, y+\bar{y}, z+\bar{z}+\frac{x \bar{y}}{2}-\frac{\bar{x} y}{2}\right) .
$$

Considerando a aplicação:

$$
\mathbb{R}^{3} \longrightarrow\left\{\left(\begin{array}{ccc}
1 & a & b \\
0 & 1 & c \\
0 & 0 & 1
\end{array}\right): a, b, c \in \mathbb{R}\right\}
$$

dada por

$$
(x, y, z) \longmapsto\left(\begin{array}{ccc}
1 & x & \frac{x y}{2} \\
0 & 1 & y \\
0 & 0 & 1
\end{array}\right),
$$

podemos verificar que ela é um isomorfismo entre $\left(\mathbb{R}^{3}, *\right)$ e um subgrupo de $G L(3, \mathbb{R})$. Para todo $\tau \in \mathbb{R}, \operatorname{com} \tau \neq 0$, a métrica Riemanniana em $\left(\mathbb{R}^{3}, *\right)$ dada por

$$
d s^{2}=d x^{2}+d y^{2}+4 \tau^{2}\left(d z+\frac{y d x-x d y}{2}\right)^{2}
$$

é invariante à esquerda e, após a mudança de coordenadas

$$
(x, y, 2 \tau z) \mapsto(x, y, z),
$$

pode ser reescrita como:

$$
d s^{2}=d x^{2}+d y^{2}+(d z+\tau(y d x-x d y))^{2} .
$$

De agora em diante, denotaremos o grupo $\left(\mathbb{R}^{3}, *\right)$ com a métrica 3.17 por $\mathbb{H}_{3}$. O lema que segue é um caso particular do Lema 3.1 .4

Lema 3.2.2. Os seguintes campos vetoriais formam uma base ortonormal de campos invariantes à esquerda em $\mathbb{H}_{3}$ :

$$
e_{1}=\partial_{x}-\tau y \partial_{z}, \quad e_{2}=\partial_{y}+\tau x \partial_{z}, \quad e_{3}=\partial_{z}
$$

A geometria de $\mathbb{H}_{3}$ pode ser descrita em termos dessa base como segue: 
1. Os campos acima satisfazem as relações de comutação:

$$
\left[e_{1}, e_{2}\right]=2 \tau e_{3}, \quad\left[e_{2}, e_{3}\right]=0, \quad\left[e_{3}, e_{1}\right]=0 .
$$

2. A conexão de Levi-Civita $\bar{\nabla}$ de $\mathbb{H}_{3}$ é dada por:

$$
\begin{aligned}
& \bar{\nabla}_{e_{1}} e_{1}=0, \quad \bar{\nabla}_{e_{1}} e_{2}=\tau e_{3}, \quad \bar{\nabla}_{e_{1}} e_{3}=-\tau e_{2}, \\
& \bar{\nabla}_{e_{2}} e_{1}=-\tau e_{3}, \quad \bar{\nabla}_{e_{2}} e_{2}=0, \quad \bar{\nabla}_{e_{2}} e_{3}=\tau e_{1}, \\
& \bar{\nabla}_{e_{3}} e_{1}=-\tau e_{2}, \quad \bar{\nabla}_{e_{3}} e_{2}=\tau e_{1}, \quad \bar{\nabla}_{e_{3}} e_{3}=0 \text {. }
\end{aligned}
$$

3. O tensor curvatura de Riemann-Christoffel $\bar{R}$ de $\mathbb{H}_{3}$ é determinado por:

$$
\begin{aligned}
\bar{R}(X, Y) Z= & -3 \tau^{2}(\langle Y, Z\rangle X-\langle X, Z\rangle Y) \\
& +4 \tau^{2}\left(\left\langle Y, e_{3}\right\rangle\left\langle Z, e_{3}\right\rangle X-\left\langle X, e_{3}\right\rangle\left\langle Z, e_{3}\right\rangle Y\right. \\
& \left.+\left\langle X, e_{3}\right\rangle\langle Y, Z\rangle e_{3}-\left\langle Y, e_{3}\right\rangle\langle X, Z\rangle e_{3}\right)
\end{aligned}
$$

onde $X, Y, Z \in \chi\left(\mathbb{H}_{3}\right)$.

Demonstração. Basta tomar $k=0$ no Lema 3.1.4.

Observação 3.2.3. O campo de Killing $e_{3}$ tem um papel importante na geometria de $\mathbb{H}_{3}$ pois suas curvas integrais são exatamente as fibras da fibração de Hopf $\pi: \mathbb{H}_{3} \longrightarrow \mathbb{R}^{2}$, dada por:

$$
\pi(x, y, z)=(x, y)
$$

\subsection{Preliminares}

Seja $F: M^{2} \longrightarrow \mathbb{H}_{3}$ uma imersão isométrica de uma superfície orientada no grupo de Heisenberg. Então, neste caso, as quatro equações de compatibilidade se tornam:

$$
\begin{gathered}
\nabla_{X} A(Y)-\nabla_{Y} A(X)-A[X, Y]=-4 \tau^{2} \cos \theta(\langle Y, T\rangle X-\langle X, T\rangle Y), \\
K=\operatorname{det} A-4 \tau^{2} \cos ^{2} \theta \\
\nabla_{X} T=\cos \theta(A(X)-\tau J X) \\
X[\cos \theta]=-\langle A(X)-\tau J X, T\rangle
\end{gathered}
$$

e o Lema 3.1.9 pode ser escrito como segue 
Lema 3.3.1. Seja $M$ uma superfície de ângulo constante $\theta$ em $\mathbb{H}_{3}$. Então:

1. a matriz do operador forma com respeito a base $\{T, J T\}$ é dada por:

$$
A=\left(\begin{array}{cc}
0 & -\tau \\
\tau & \lambda
\end{array}\right),
$$

onde $\lambda \in C^{\infty}(M)$.

2. A conexão de Levi-Civita da superfície é determinada por:

$$
\begin{array}{ll}
\nabla_{T} T=-2 \tau \cos \theta J T, & \nabla_{J T} T=\lambda \cos \theta J T, \\
\nabla_{T} J T=2 \tau \cos \theta T, & \nabla_{J T} J T=-\lambda \cos \theta T .
\end{array}
$$

3. A curvatura Gaussiana da superfície $M$ é a constante negativa dada por:

$$
K=-4 \tau^{2} \cos ^{2} \theta
$$

4. A função $\lambda$ satisfaz a equação:

$$
T[\lambda]+\lambda^{2} \cos \theta+4 \tau^{2} \cos ^{3} \theta=0 .
$$

\subsection{Superfícies de ângulo constante em $\mathbb{H}_{3}$}

Usando o Lema 3.3.1 provaremos o teorema principal deste capítulo que da a classificação (local) completa das superfícies de ângulo constante em $\mathbb{H}_{3}$, ou seja:

Teorema 3.4.1. Seja $M$ uma superfície de ângulo constante em $\mathbb{H}_{3}$. Então $M$ é isométrica a um aberto de alguma das seguintes superfícies:

1. um cilindro de Hopf;

2. a superfície dada por:

$$
\begin{aligned}
F(u, v)= & \left(\frac{1}{2 \tau} \tan \theta \sin u+f_{1}(v),-\frac{1}{2 \tau} \tan \theta \cos u+f_{2}(v),\right. \\
& \left.-\frac{1}{4 \tau} \tan ^{2} \theta u-\frac{1}{2} \tan \theta \cos u f_{1}(v)-\frac{1}{2} \tan \theta \sin u f_{2}(v)-\tau f_{3}(v)\right),
\end{aligned}
$$


com

$$
\left(f_{1}^{\prime}\right)^{2}+\left(f_{2}^{\prime}\right)^{2}=\sin ^{2} \theta \quad \text { e } \quad f_{3}^{\prime}(v)=f_{1}^{\prime}(v) f_{2}(v)-f_{1}(v) f_{2}^{\prime}(v)
$$

onde $\theta$ denota o ângulo constante.

Demonstração. Suponhamos que a superfície $M$ faz ângulo constante $\theta=\pi / 2$ com $e_{3}$. Neste caso, teremos que $e_{3}$ será tangente à superfície em todo ponto. Logo $M$ é um cilindro de Hopf.

Observemos agora que $\theta$ deve ser diferente de zero, pois caso fosse nulo, teríamos uma contradição com o item 1, do Lema 3.2.2. Assim, a partir de agora podemos assumir que $\theta \in(0, \pi / 2)$. Como $N$ é unitário, decompondo ele na base $\left\{e_{1}, e_{2}, e_{3}\right\}$ existirá uma função diferenciável $\phi$, localmente definida em $M$, tal que:

$$
N=\sin \theta \cos \phi e_{1}+\sin \theta \sin \phi e_{2}+\cos \theta e_{3} .
$$

Com isso, obtemos

$$
\begin{gathered}
T=-\sin \theta\left(\cos \theta \cos \phi e_{1}+\cos \theta \sin \phi e_{2}-\sin \theta e_{3}\right), \\
J T=\sin \theta\left(\sin \phi e_{1}-\cos \phi e_{2}\right) .
\end{gathered}
$$

Temos ainda, pela fórmula de Weingarten combinada com o item 2 , do Lema 3.2.2 e as equações (3.19) e (3.20), que o operador forma $A$ satisfaz

$$
\begin{gathered}
A(T)=-\bar{\nabla}_{T} N=\left(T[\phi]-\tau \sin ^{2} \theta+\tau \cos ^{2} \theta\right) J T, \\
A(J T)=-\bar{\nabla}_{J T} N=-\tau T+(J T)[\phi] J T .
\end{gathered}
$$

Comparando essas equações com o item 1, do Lema 3.3.1 temos

$$
\left\{\begin{array}{l}
T[\phi]=-2 \tau \cos ^{2} \theta \\
(J T)[\phi]=\lambda
\end{array}\right.
$$

Observe que a condição de integrabilidade para esse sistema de equações diferenciais é exatamente o item 4, do Lema 3.3.1. A fim de resolver o sistema (3.21), vamos escolher as coordenadas $(u, v)$ em $M$ de forma que:

$$
\partial_{u}=T \quad \text { e } \quad \partial_{v}=a T+b J T,
$$


onde $a, b \in C^{\infty}(M)$. Assim a condição $\left[\partial_{u}, \partial_{v}\right]=0$ é equivalente ao seguinte sistema de equações:

$$
\left\{\begin{array}{l}
\partial_{u} a=-2 \tau b \cos \theta, \\
\partial_{u} b=b \lambda \cos \theta
\end{array}\right.
$$

A equação diferencial do item4 do Lema 3.3.1é agora equivalente a

$$
\lambda_{u}+\lambda^{2} \cos ^{2} \theta+4 \tau^{2} \cos ^{3} \theta=0,
$$

cuja solução geral dada por

$$
\lambda(u, v)=2 \tau \cos \theta \tan \left(\varphi(v)-2 \tau \cos ^{2} \theta u\right)
$$

onde $\varphi \in C^{\infty}(\mathbb{R})$. Podemos agora resolver o sistema (3.22). Como estamos interessados em um único sistema de coordenadas para a superfície $M$, precisamos somente de uma solução para $a$ e $b$, por exemplo:

$$
\begin{aligned}
& a(u, v)=\frac{1}{\cos \theta} \sin \left(\varphi(v)-2 \tau \cos ^{2} \theta u\right), \\
& b(u, v)=\cos \left(\varphi(v)-2 \tau \cos ^{2} \theta u\right)
\end{aligned}
$$

Portanto, o sistema (3.21) é agora equivalente a

$$
\left\{\begin{array}{l}
\phi_{u}=-2 \tau \cos ^{2} \theta \\
\phi_{v}=0
\end{array}\right.
$$

o qual tem solução geral dada por:

$$
\phi(u, v)=-2 \tau \cos ^{2} \theta u+c, \quad c \in \mathbb{R} .
$$

Para concluir a demonstração, resta somente integrar a distribuição gerada por $T$ e $J T$. Denotando a parametrização de $M$ por:

$$
\begin{aligned}
F: U \subseteq \mathbb{R}^{2} \longrightarrow M \subset \mathbb{H}_{3} \\
(u, v) \longmapsto F(u, v)=\left(F_{1}(u, v), F_{2}(u, v), F_{3}(u, v)\right),
\end{aligned}
$$

das expressões de $T$ e $J T$ na base $\left\{e_{1}, e_{2}, e_{3}\right\}$ e pela escolha das coordenadas $(u, v)$ que:

$$
\begin{aligned}
\left(\partial_{u} F_{1}, \partial_{u} F_{2}, \partial_{u} F_{3}\right)= & -\sin \theta\left(\cos \theta \cos \phi e_{1}+\cos \theta \sin \phi e_{2}-\sin \theta e_{3}\right), \\
\left(\partial_{v} F_{1}, \partial_{v} F_{2}, \partial_{v} F_{3}\right)= & \sin \theta\left[(-a \cos \theta \cos \phi+b \sin \phi) e_{1}\right. \\
& \left.-(a \cos \theta \sin \phi+b \cos \phi) e_{2}+a \sin \theta e_{3}\right] .
\end{aligned}
$$


Além disso, no ponto $F(u, v)$, temos:

$$
e_{1}=\left(1,0,-\tau F_{2}\right), \quad e_{2}=\left(0,1, \tau F_{1}\right), \quad e_{3}=(0,0,1)
$$

e $a, b, \phi$ são dados por (3.24), (3.25) e (3.27), respectivamente. Uma integração direta das equações (3.28) e (3.29), seguida pela reparametrização:

$$
-2 \tau \cos ^{2} \theta u+c \mapsto u
$$

resulta na parametrização dada no enunciado do teorema, onde $f_{1}(v)$ e $f_{2}(v)$ são primitivas das funções

$$
\sin \theta \sin (c-\varphi(v)) \quad \text { e } \quad \sin \theta \cos (c-\varphi(v))
$$

respectivamente.

Segue um exemplo de uma superfície, não trivial, de ângulo constante em $\mathbb{H}_{3}$.

Exemplo 3.4.2. Tomemos

$$
f_{1}(v)=f_{2}(v)=0 \quad \text { e } \quad f_{3}(v)=\frac{1}{\sqrt{2}} v
$$

Então, segue do Teorema 3.4.1 que a superfície dada por:

$$
F(u, v)=\left(\frac{1}{2 \tau} \sin u,-\frac{1}{2 \tau} \cos u+\frac{1}{\sqrt{2}} v,-\frac{1}{4 \tau} u-\frac{1}{2 \sqrt{2}} v \sin u\right)
$$

é uma superfície de ângulo constante em $\mathbb{H}_{3}, \operatorname{com} \theta=\pi / 4$. Esta é uma superfície regrada com curva base uma hélice.

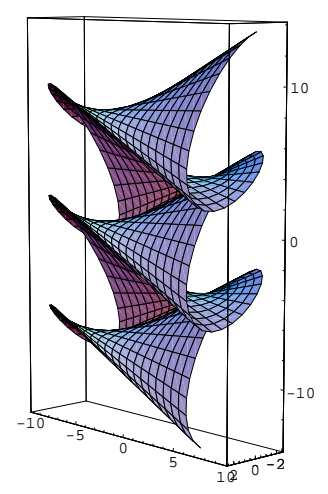

Figura 3.1: Superfície de ângulo constante no grupo de Heisenberg $\operatorname{com} \tau=2$. 


\begin{tabular}{l|l|}
\hline CAPÍtulo \\
\cline { 2 - 2 } & 4 \\
\hline
\end{tabular}

\section{Superfícies de ângulo constante nas esferas de Berger}

As esferas de Berger $\mathbb{S}_{\epsilon}^{3}, \epsilon>0$, são espaços Riemannianos homogêneos difeomorfos à esfera tridimensional $\mathbb{S}^{3}$. Esses espaços foram descobertos por M. Berger (ver [4]) em sua classificação de todas as variedades Riemannianas, normais, simplesmente conexas, com curvatura seccional positiva e não constante. Suas métricas são obtidas a partir da métrica usual de $\mathbb{S}^{3}$ através uma deformação ao longo das fibras do fibrado de Hopf $\psi: \mathbb{S}^{3} \longrightarrow \mathbb{S}^{2}$ por $\epsilon$. A importância desse espaços está ligada ao fato do que eles servem como contra-exemplo para varias conjecturas (ver [3, 28, 33, 35]).

Neste capítulo iremos apresentar de forma detalhada os resultados obtidos por Montaldo e Onnis em [24] sobre a classificação das superfícies de ângulo constante nas esferas de Berger 3-dimensionais, que são definidas a partir do fibrado de Hopf como descrito na seção que segue. 


\subsection{As esferas de Berger}

Sejam

$$
\mathbb{S}^{2}\left(\frac{1}{2}\right)=\left\{(z, t) \in \mathbb{C} \times \mathbb{R} ;|z|^{2}+t^{2}=\frac{1}{4}\right\}
$$

$\mathrm{e}$

$$
\mathbb{S}^{3}=\left\{(z, w) \in \mathbb{C}^{2} ;|z|^{2}+|w|^{2}=1\right\}
$$

as esferas 2-dimensional e 3-dimensional usuais (de curvatura constante 4 e 1 , respectivamente). Então chama-se aplicação de Hopf a submersão Riemanniana $\psi: \mathbb{S}^{3} \longrightarrow \mathbb{S}^{2}(1 / 2)$, definida por:

$$
\psi(z, w)=\frac{1}{2}\left(2 z \bar{w},|z|^{2}-|w|^{2}\right) .
$$

Consideremos agora os seguintes campos vetoriais sobre $\mathbb{S}^{3}$ :

$$
X_{1}(z, w)=(i z, i w), \quad X_{2}(z, w)=(-i \bar{w}, i \bar{z}), \quad X_{3}(z, w)=(-\bar{w}, \bar{z}) .
$$

Temos que eles paralelizam $\mathbb{S}^{3}$, sendo que $X_{1}$ é vertical (i.e. $\left.d \psi\left(X_{1}\right)=0\right)$ e $X_{2}, X_{3}$ são horizontais. O campo $X_{1}$ é chamado campo vetorial de Hopf. A esfera de Berger $\mathbb{S}_{\epsilon}^{3}, \epsilon>0$, é a esfera $\mathbb{S}^{3}$ munida com a métrica:

$$
g_{\epsilon}(X, Y)=\langle X, Y\rangle+\left(\epsilon^{2}-1\right)\left\langle X, X_{1}\right\rangle\left\langle Y, X_{1}\right\rangle
$$

onde $\langle.,$.$\rangle representa a métrica canônica de \mathbb{S}^{3}$. Observe que o campo vetorial de Hopf $X_{1}$ é um campo de Killing de norma constante e igual a $\epsilon$. Assim, definimos uma superfície de ângulo constante em $\mathbb{S}_{\epsilon}^{3}$ como uma superfície orientada tal que sua normal unitária $N$ satisfaz

$$
\left|g_{\epsilon}(X, N)\right|=\epsilon \cos \theta
$$

para um $\theta \in[0, \pi / 2]$ fixado.

Teorema 4.1.1. Seja $M^{2}$ uma superfície na esfera de Berger $\mathbb{S}_{\epsilon}^{3}$ de ângulo constante $\theta \neq \pi / 2$. Então existe um sistema de coordenadas locais em $M^{2}$ tal que seu vetor posição em $\mathbb{R}^{4}$ é dado por:

$$
F(u, v)=A(v) \beta(u)
$$

onde

$$
\beta(u)=\left(\sqrt{c_{1}} \cos \left(\alpha_{1} u\right), \sqrt{c_{1}} \sin \left(\alpha_{1} u\right), \sqrt{c_{2}} \cos \left(\alpha_{2} u\right), \sqrt{c_{2}} \sin \left(\alpha_{2} u\right)\right)
$$


é uma geodésica no toro $\mathbb{S}^{1}\left(\sqrt{c_{1}}\right) \times \mathbb{S}^{1}\left(\sqrt{c_{2}}\right)$, com:

$$
c_{1,2}=\frac{1}{2} \pm \epsilon \frac{\cos \theta}{2 \sqrt{B}}, \quad \alpha_{1}=\frac{2 B}{\epsilon} c_{2}, \quad \alpha_{2}=\frac{2 B}{\epsilon} c_{1}, \quad B=1+\left(\epsilon^{2}-1\right) \cos ^{2} \theta,
$$

enquanto $A(v)=A\left(\xi, \xi_{1}, \xi_{2}, \xi_{3}\right)(v)$ é uma família a 1-parâmetro de matrizes $4 \times 4$ que comutam com a estrutura complexa $J_{1}$ de $\mathbb{R}^{4}$ (como será descrito em (4.46)), com $\xi=$ constante $e$

$$
\cos ^{2}\left(\xi_{1}(v)\right) \xi_{2}^{\prime}(v)-\sin ^{2}\left(\xi_{2}(v)\right) \xi_{3}^{\prime}(v)=0
$$

Reciprocamente, a parametrização $F(u, v)=A(v) \beta(v)$, com $\beta(u)$ e $A(v)$ como acima, define uma superfície na esfera de Berger $\mathbb{S}_{\epsilon}^{3}$ de ângulo constante $\theta \neq \pi / 2$.

\subsubsection{Estrutura Riemanniana das esfera de Berger}

Considere os seguintes campos

$$
E_{1}=\epsilon^{-1} X_{1}, \quad E_{2}=X_{2}, \quad E_{3}=X_{3},
$$

que formam uma base ortonormal em $\mathbb{S}_{\epsilon}^{3}$. Primeiramente observe que eles satisfazem as relações:

$$
\begin{aligned}
& {\left[E_{1}, E_{2}\right]=2 \epsilon^{-1} E_{3},} \\
& {\left[E_{1}, E_{3}\right]=-2 \epsilon^{-1} E_{2},} \\
& {\left[E_{2}, E_{3}\right]=2 \epsilon E_{1} .}
\end{aligned}
$$

A conexão de Levi-Civita $\nabla^{\epsilon}$ de $\left(\mathbb{S}_{\epsilon}^{3}, g_{\epsilon}\right)$ é dada por:

$$
\begin{array}{lll}
\nabla_{E_{1}}^{\epsilon} E_{1}=0, & \nabla_{E_{2}}^{\epsilon} E_{2}=0, & \nabla_{E_{3}}^{\epsilon} E_{3}=0, \\
\nabla_{E_{1}}^{\epsilon} E_{2}=\epsilon^{-1}\left(2-\epsilon^{2}\right) E_{3}, & & \nabla_{E_{1}}^{\epsilon} E_{3}=-\epsilon^{-1}\left(2-\epsilon^{2}\right) E_{2}, \\
\nabla_{E_{2}}^{\epsilon} E_{1}=-\epsilon E_{3}, & \nabla_{E_{3}}^{\epsilon} E_{1}=\epsilon E_{2}, & \nabla_{E_{3}}^{\epsilon} E_{2}=-\epsilon E_{1}=-\nabla_{E_{2}}^{\epsilon} E_{3} .
\end{array}
$$

Além disso, as componentes do tensor curvatura são dadas por:

$$
\begin{array}{lll}
R\left(E_{1}, E_{2}\right) E_{1}=-\epsilon^{2} E_{2}, & R\left(E_{1}, E_{2}\right) E_{2}=\epsilon^{2} E_{1}, & R\left(E_{1}, E_{2}\right) E_{3}=0, \\
R\left(E_{1}, E_{3}\right) E_{1}=-\epsilon^{2} E_{3}, & R\left(E_{1}, E_{3}\right) E_{2}=0, & R\left(E_{1}, E_{3}\right) E_{3}=\epsilon^{2} E_{1}, \\
R\left(E_{2}, E_{3}\right) E_{1}=0, & R\left(E_{2}, E_{3}\right) E_{2}=\left(3 \epsilon^{2}-4\right) E_{3}, & R\left(E_{2}, E_{3}\right) E_{3}=\left(4-3 \epsilon^{2}\right) E_{2} .
\end{array}
$$

Por fim, a curvatura seccional da esfera de Berger é dada por:

$$
K\left(E_{1}, E_{2}\right)=K\left(E_{1}, E_{3}\right)=\epsilon^{2} \quad \text { e } \quad K\left(E_{2}, E_{3}\right)=4-3 \epsilon^{2} .
$$




\subsection{Superfícies de ângulo constante}

Seja $M^{2}$ uma superfície orientada de ângulo constante em $\mathbb{S}_{\epsilon}^{3}$ e seja $N$ seu campo normal unitário, então por definição temos:

$$
\left|g_{\epsilon}\left(E_{1}, N\right)\right|=\cos \theta
$$

para algum $\theta \in[0, \pi / 2]$ fixado. Note que $\theta \neq 0$, pois se isso ocorresse, teríamos que os campos $E_{2}$ e $E_{3}$ seriam tangentes à superfície $M^{2}$, o que é um absurdo pois a distribuição horizontal da aplicação de Hopf não é integrável. Observe, também, que se $\theta=\pi / 2$ teríamos que $E_{1}$ seria sempre tangente a $M$ e, neste caso, $M$ seria um cilindro de Hopf. Assim, daqui em diante, assumiremos que $\theta \neq 0, \pi / 2$.

Dados $X, Y \in \chi(M)$, lembramos que as fórmulas de Gauss e Weingarten são dadas (respectivamente) por:

$$
\begin{aligned}
\nabla_{X}^{\epsilon} Y & =\nabla_{X} Y+h(X, Y), \\
\nabla_{X}^{\epsilon} N & =-A(X),
\end{aligned}
$$

onde $A$ indica o operador forma de $M$ em $\mathbb{S}_{\epsilon}^{3}, \nabla$ a conexão de Levi-Civita induzida em $M$ e $h$ a segunda forma fundamental de $M$ em $\mathbb{S}_{\epsilon}^{3}$. Decompondo $E_{1}$ em parte tangente e parte normal, e levando em consideração a equação (4.7), temos:

$$
E_{1}=T+\cos \theta N
$$

onde $g_{\epsilon}(T, T)=\sin ^{2} \theta$. Assim, para todo $X \in \chi(M)$, resulta

$$
\begin{aligned}
\nabla_{X}^{\epsilon} E_{1} & =\nabla_{X}^{\epsilon} T+\cos \theta \nabla_{X}^{\epsilon} N \\
& =\nabla_{X} T+h(X, T)+\cos \theta \nabla_{X}^{\epsilon} N \\
& =\nabla_{X} T+h(X, T)-\cos \theta A(X) .
\end{aligned}
$$

Como

$$
h(X, T)=g_{\epsilon}(h(X, T), N) N,
$$

segue que

$$
\nabla_{X}^{\epsilon} E_{1}=\nabla_{X} T+g_{\epsilon}(A(X), T) N-\cos \theta A(X) .
$$


Por outro lado, escrevendo $X=\sum_{i=1}^{3} X_{i} E_{i}$, temos:

$$
\begin{aligned}
\nabla_{X}^{\epsilon} E_{1} & =\epsilon\left(X_{3} E_{2}-X_{2} E_{3}\right) \\
& =\epsilon g_{\epsilon}(J X, T) N-\epsilon \cos \theta J X,
\end{aligned}
$$

onde $J X$ denota a rotação de um ângulo $\pi / 2$ em $T M$. Identificando as componentes tangente e normal de (4.10) e (4.11) respectivamente, obtemos:

$$
\nabla_{X} T=\cos \theta(A(X)-\epsilon J X)
$$

e

$$
g_{\epsilon}(A(X)-\epsilon J X, T)=0 .
$$

Lema 4.2.1. Seja $M^{2}$ uma superfície orientada de ângulo constante $\theta$ em $\mathbb{S}_{\epsilon}^{3}$. Então:

1. com respeito a base $\{T, J T\}$, a matriz associada ao operador forma A é dada por

$$
A=\left(\begin{array}{cc}
0 & -\epsilon \\
-\epsilon & \lambda
\end{array}\right),
$$

onde $\lambda \in C^{\infty}(M)$.

2. A conexão de Levi-Civita $\nabla$ de $M$ é dada por:

$$
\begin{gathered}
\nabla_{T} T=-2 \epsilon \cos \theta J T, \quad \nabla_{J T} T=\lambda \cos \theta J T, \\
\nabla_{T} J T=2 \epsilon \cos \theta T, \quad \nabla_{J T} J T=-\lambda \cos \theta T .
\end{gathered}
$$

3. A curvatura Gaussiana de $M$ é constante e é dada por:

$$
K=4\left(1-\epsilon^{2}\right) \cos ^{2} \theta
$$

4. A função $\lambda$, definida em 1 , satisfaz a seguinte equação:

$$
T[\lambda]+\lambda^{2} \cos \theta+4\left(\epsilon^{2}-1\right) \cos ^{3} \theta+4 \cos \theta=0 .
$$

Demonstração. 1. Das equações (4.12) e (4.13) temos que:

$$
g_{\epsilon}(A(X), T)=\epsilon g_{\epsilon}(J X, T),
$$


$\log 0$

$$
\begin{array}{ll}
g_{\epsilon}(A(T), T)=0, & g_{\epsilon}(A(T), J T)=-\epsilon \sin ^{2} \theta, \\
g_{\epsilon}(A(J T), T)=-\epsilon \sin ^{2} \theta, & g_{\epsilon}(A(J T), J T)=\bar{\lambda} .
\end{array}
$$

Como

$$
\|T\|_{g_{\epsilon}}^{2}=\|J T\|_{g_{\epsilon}}^{2}=\sin ^{2} \theta
$$

tomando $\lambda=\frac{\bar{\lambda}}{\sin ^{2} \theta}$, temos o resultado.

2. Da equação (4.12) segue que:

$$
\begin{aligned}
\nabla_{T} T & =-2 \epsilon \cos \theta J T, \\
\nabla_{J T} T & =\lambda \cos \theta J T .
\end{aligned}
$$

Usando (4.15) e $g_{\epsilon}(J T, T)=0$, resulta

$$
g_{\epsilon}\left(\nabla_{T} J T, T\right)=-g_{\epsilon}\left(\nabla_{T} T, J T\right)=2 \epsilon \frac{\cos \theta}{\sin ^{2} \theta} .
$$

Logo

$$
\nabla_{T} J T=2 \epsilon \cos \theta T
$$

De forma análoga, obtemos que:

$$
\nabla_{J T} J T=-\lambda \cos \theta T
$$

3. Da equação de Gauss e do primeiro item temos que a curvatura de Gauss de $M$ é dada por:

$$
\begin{aligned}
K & =\operatorname{det} A+\epsilon^{2}+4\left(1-\epsilon^{2}\right) \cos ^{2} \theta \\
& =4\left(1-\epsilon^{2}\right) \cos ^{2} \theta
\end{aligned}
$$

4. Sabemos que

$$
K(J T, T)=\frac{\langle R(J T, T) T, J T\rangle}{\|J T\|^{2}\|T\|^{2}}=4\left(1-\epsilon^{2}\right) \cos ^{2} \theta .
$$

Como

$$
R(J T, T) T=-\cos \theta T[\lambda] J T-\lambda^{2} \cos ^{2} \theta J T-4 \epsilon^{2} \cos ^{2} \theta J T,
$$

segue que

$$
\begin{aligned}
0 & =-\cos \theta T[\lambda]-\lambda^{2} \cos ^{2} \theta-4 \epsilon^{2} \cos ^{2} \theta-4\left(1-\epsilon^{2}\right) \cos ^{2} \theta \sin ^{2} \theta \\
& =\cos \theta T[\lambda]+4\left(\epsilon^{2}-1\right) \cos ^{4} \theta+\lambda^{2} \cos ^{2} \theta+4 \cos ^{2} \theta .
\end{aligned}
$$


Sendo $\cos \theta \neq 0$, obtemos

$$
T[\lambda]+4\left(\epsilon^{2}-1\right) \cos ^{3} \theta+\lambda^{2} \cos \theta+4 \cos \theta=0 .
$$

Observação 4.2.2. Se uma superfície de ângulo constante é mínima (isto é, $\operatorname{tr} A=0$ ), então $\theta=\pi / 2$. De fato, neste caso temos que $\lambda=0$, e assim, pela equação (4.14) resulta que $\cos \theta=0$, ou seja, $\theta=\pi / 2$.

Agora, de (4.7) temos que existe uma função $\varphi \in C^{\infty}(M)$ tal que:

$$
N=\cos \theta E_{1}+\sin \theta \cos \varphi E_{2}+\sin \theta \sin \varphi E_{3} .
$$

Assim,

$$
T=E_{1}-\cos \theta N=\sin \theta\left(\sin \theta E_{1}-\cos \theta \cos \varphi E_{2}-\cos \theta \sin \varphi E_{3}\right)
$$

$\mathrm{e}$

$$
J T=N \times T=\sin \theta\left(\sin \varphi E_{2}-\cos \varphi E_{3}\right) .
$$

Pela fórmula de Weingarten segue que

$$
A(T)=\left(T[\varphi]+\epsilon^{-1}\left(2-\epsilon^{2}\right) \sin ^{2} \theta+\epsilon \cos ^{2} \theta\right) J T
$$

$\mathrm{e}$

$$
A(J T)=J T[\varphi] J T-\epsilon T .
$$

Comparando essas duas últimas equações com o item 1, do Lema 4.2.1 temos:

$$
\left\{\begin{array}{l}
J T[\varphi]=\lambda, \\
T[\varphi]=-2 \epsilon^{-1} B,
\end{array}\right.
$$

onde

$$
B=1+\left(\epsilon^{2}-1\right) \cos ^{2} \theta .
$$

Como

$$
[T, J T]=\cos \theta(2 \epsilon T-\lambda J T),
$$

a condição de compatibilidade do sistema (4.20) é dada por:

$$
\left(\nabla_{T} J T-\nabla_{J T} T\right)[\varphi]=[T, J T][\varphi]=T(J T[\varphi])-J T(T[\varphi])
$$


e é equivalente à equação (4.14).

Ao fim de resolver o sistema (4.20), vamos escolher um sistema de coordenadas locais $(u, v)$ em $M$ de forma que:

$$
\partial_{u}=T
$$

Como $\partial_{v}$ é também tangente a $M$, podemos escrever

$$
\partial_{v}=a T+b J T
$$

onde $a=a(u, v)$ e $b=b(u, v)$ são funções definidas (localmente) em $M$. Agora, como

$$
0=\left[\partial_{u}, \partial_{v}\right]=\left(a_{u}+2 \epsilon b \cos \theta\right) T+\left(b_{u}-b \lambda \cos \theta\right) J T,
$$

obtemos que

$$
\left\{\begin{array}{l}
a_{u}=-2 \epsilon b \cos \theta, \\
b_{u}=b \lambda \cos \theta .
\end{array}\right.
$$

Além disso, podemos reescrever a equação (4.14) na forma

$$
\lambda_{u}+\cos \theta \lambda^{2}+4\left(\epsilon^{2}-1\right) \cos ^{3} \theta+4 \cos \theta=0,
$$

a qual, após integração, tem solução dada por:

$$
\lambda(u, v)=2 \sqrt{B} \tan (\eta(v)-2 \cos \theta \sqrt{B} u),
$$

onde $\eta \in C^{\infty}(\mathbb{R})$. Substituindo a equação acima no sistema (4.24) obtemos:

$$
b_{u}=2 b \sqrt{B} \tan (\eta(v)-2 \cos \theta \sqrt{B} u) \cos \theta
$$

$\mathrm{e}$

$$
a_{u}=-2 \epsilon \cos \theta \cos (\eta(v)-2 \cos \theta \sqrt{B} u) .
$$

Consequentemente

$$
\left\{\begin{array}{l}
a(u, v)=\frac{\epsilon}{\sqrt{B}} \sin (\eta(v)-2 \cos \theta \sqrt{B} u), \\
b(u, v)=\cos (\eta(v)-2 \cos \theta \sqrt{B} u)
\end{array}\right.
$$

Além disso, a partir da equação (4.20) obtemos o sistema

$$
\left\{\begin{array}{l}
\varphi_{u}=-2 \epsilon^{-1} B \\
\varphi_{v}=0
\end{array}\right.
$$


o qual tem solução dada por:

$$
\varphi(u, v)=-2 \epsilon^{-1} B u+c,
$$

onde $c \in \mathbb{R}$.

Temos agora a seguinte caracterização do vetor posição de uma superfície de ângulo constante com respeito as coordenadas locais $(u, v)$ descritas em (4.22) e (4.23).

Proposição 4.2.3. Seja $M^{2}$ uma superfície em $\mathbb{S}_{\epsilon}^{3}$ de ângulo constante $\theta$. Então, com respeito as coordenadas locais $(u, v)$ definidas em (4.22) e (4.23), o vetor posição $F$ de $M^{2}$ satisfaz a equação:

$$
\frac{\partial^{4} F}{\partial u^{4}}+\left(\tilde{b}^{2}-2 \tilde{a}\right) \frac{\partial^{2} F}{\partial u^{2}}+\tilde{a}^{2} F=0
$$

onde

$$
\tilde{a}=\epsilon^{-2} \sin ^{2} \theta B, \quad \tilde{b}=-2 \epsilon^{-1} B
$$

$e$

$$
B=1+\left(\epsilon^{2}-1\right) \cos ^{2} \theta .
$$

Demonstração. Seja $M^{2}$ uma superfície de ângulo constante em $\mathbb{S}_{\epsilon}^{3} \subset \mathbb{R}^{4}$ e seja $F$ o vetor posição de $M^{2}$. Então, com respeito as coordenadas locais $(u, v)$ definidas em (4.22) e (4.23), podemos escrever

$$
F(u, v)=\left(F_{1}(u, v), F_{2}(u, v), F_{3}(u, v), F_{4}(u, v)\right) .
$$

Pela definição e levando em consideração a equação (4.16) temos:

$$
\begin{aligned}
\partial_{u} F & =\left(\partial_{u} F_{1}, \partial_{u} F_{2}, \partial_{u} F_{3}, \partial_{u} F_{4}\right)=T \\
& =\sin \theta\left(\sin \theta E_{1 \mid F(u, v)}-\cos \theta \cos \varphi E_{2 \mid F(u, v)}-\cos \theta \sin \varphi E_{3 \mid F(u, v)}\right) .
\end{aligned}
$$

Usando, então, as expressões de $E_{1}, E_{2}$, e $E_{3}$ com respeito aos campos coordenados de $\mathbb{R}^{4}$, obtemos:

$$
\left\{\begin{array}{l}
\partial_{u} F_{1}=\sin \theta\left(\epsilon^{-1} \sin \theta F_{2}+\cos \theta \cos \varphi F_{4}+\cos \theta \sin \varphi F_{3}\right), \\
\partial_{u} F_{2}=\sin \theta\left(\epsilon^{-1} \sin \theta F_{1}+\cos \theta \cos \varphi F_{3}-\cos \theta \sin \varphi F_{4}\right), \\
\partial_{u} F_{3}=-\sin \theta\left(\epsilon^{-1} \sin \theta F_{4}+\cos \theta \cos \varphi F_{2}+\cos \theta \sin \varphi F_{1}\right), \\
\partial_{u} F_{4}=\sin \theta\left(\epsilon^{-1} \sin \theta F_{3}-\cos \theta \cos \varphi F_{1}+\cos \theta \sin \varphi F_{2}\right) .
\end{array}\right.
$$


Derivando as equações (4.32) com relação a $u$, achamos que existem constantes $\tilde{a}$ e $\tilde{b}$ tais que:

$$
\left\{\begin{array}{l}
\left(F_{1}\right)_{u u}=\tilde{a} F_{1}+\tilde{b}\left(F_{2}\right)_{u}, \\
\left(F_{2}\right)_{u u}=\tilde{a} F_{2}+\tilde{b}\left(F_{1}\right)_{u}, \\
\left(F_{3}\right)_{u u}=\tilde{a} F_{3}+\tilde{b}\left(F_{4}\right)_{u} \\
\left(F_{4}\right)_{u u}=\tilde{a} F_{4}+\tilde{b}\left(F_{3}\right)_{u},
\end{array}\right.
$$

onde, usando (4.27), obtemos:

$$
\tilde{a}=-\frac{\epsilon^{-1} \sin ^{2} \theta}{2} \varphi_{u}=\epsilon^{-2} \sin ^{2} \theta B, \quad \tilde{b}=\varphi_{u}=-2 \epsilon^{-1} B .
$$

Derivando duas vezes as equações (4.33) e usando que:

$$
\left\{\begin{array}{l}
\tilde{b} \partial_{u} F_{2}=\partial_{u}^{2} F_{1}-\tilde{a} F_{1}, \\
\tilde{b} \partial_{u} F_{1}=\tilde{a} F_{2}-\partial_{u}^{2} F_{2}, \\
\tilde{b} \partial_{u} F_{4}=\partial_{u}^{2} F_{3}-\tilde{a} F_{3}, \\
\tilde{b} \partial_{u} F_{3}=\tilde{a} F_{4}-\partial_{u}^{2} F_{4},
\end{array}\right.
$$

segue que o vetor posição de $M$ satisfaz a equação 4.29).

Corolário 4.2.4. Seja $M^{2}$ uma superfície em $\mathbb{S}_{\epsilon}^{3}$ de ângulo constante $\theta$. Então, com respeito as coordenadas locais $(u, v)$ em $M$ definidas por (4.22) e (4.23), o vetor posição $F$ é dado por:

$$
F(u, v)=\cos \left(\alpha_{1} u\right) g^{1}(v)+\sin \left(\alpha_{1} u\right) g^{2}(v)+\cos \left(\alpha_{2} u\right) g^{3}(v)+\sin \left(\alpha_{2} u\right) g^{4}(v),
$$

onde

$$
\alpha_{1,2}=\frac{1}{\epsilon}(B \pm \epsilon \sqrt{B} \cos \theta)
$$

são constantes reais e $g^{i}(v), i \in\{1,2,3,4\}$, são campos vetoriais de $\mathbb{R}^{4}$, mutualmente ortogonais, que dependem somente de $v$ tais que:

$$
\begin{aligned}
& g_{11}=\left\langle g^{1}(v), g^{1}(v)\right\rangle=g_{22}=\left\langle g^{2}(v), g^{2}(v)\right\rangle=\frac{\epsilon}{2 B} \alpha_{2}, \\
& g_{33}=\left\langle g^{3}(v), g^{3}(v)\right\rangle=g_{44}=\left\langle g^{4}(v), g^{4}(v)\right\rangle=\frac{\epsilon}{2 B} \alpha_{1} .
\end{aligned}
$$

Demonstração. Notemos que a equação (4.29) pode ser vista como uma EDO na variável $u$ e com coeficientes constantes. Assim, por uma integração direta a partir do polinômio característico e levando em consideração que as constantes de integração dependem de $v$, temos a solução

$$
F(u, v)=\cos \left(\alpha_{1} u\right) g^{1}(v)+\sin \left(\alpha_{1} u\right) g^{2}(v)+\cos \left(\alpha_{2} u\right) g^{3}(v)+\sin \left(\alpha_{2} u\right) g^{4}(v),
$$


onde

$$
\alpha_{1,2}=\sqrt{\frac{\tilde{b}^{2}-2 \tilde{a} \pm \sqrt{\tilde{b}^{4}-4 \tilde{a} \tilde{b}^{2}}}{2}} .
$$

Agora, como $\|F\|^{2}=1$, escrevendo

$$
\partial_{u} F=\left(\partial_{u} F_{1}, \partial_{u} F_{2}, \partial_{u} F_{3}, \partial_{u} F_{4}\right),
$$

e considerando as equações (4.29), (4.32) e (4.33), obtemos que o vetor posição $F$ e suas derivadas satisfazem as relações:

$$
\begin{array}{lll}
\langle F, F\rangle=1, & \left\langle F_{u}, F_{u}\right\rangle=\epsilon^{-2} B \sin ^{2} \theta, & \left\langle F, F_{u}\right\rangle=0, \\
\left\langle F_{u}, F_{u u}\right\rangle=0, & \left\langle F_{u u}, F_{u u}\right\rangle=D, & \left\langle F, F_{u u}\right\rangle=-\epsilon^{-2} B \sin ^{2} \theta, \\
\left\langle F_{u}, F_{\text {uuu }}\right\rangle=-D, & \left\langle F_{\text {uu }}, F_{\text {uuu }}\right\rangle=0, & \left\langle F, F_{\text {uuu }}\right\rangle=0, \\
\left\langle F_{\text {uuu }}, F_{\text {uuu }}\right\rangle=E, &
\end{array}
$$

onde

$$
D=\epsilon^{-2} B \tilde{b}^{2} \sin ^{2} \theta-3 \tilde{a}^{2}, \quad E=\left(\tilde{b}^{2}-2 \tilde{a}\right) D-\epsilon^{-2} B \tilde{a}^{2} \sin ^{2} \theta .
$$

Calculando agora $F$ e suas derivadas no ponto $(0, v)$ temos:

$$
\begin{array}{rr}
F(0, v)=g^{1}(v)+g^{3}(v), & F_{u u}(0, v)=-\alpha_{1}^{2} g^{1}(v)-\alpha_{2}^{2} g^{3}(v) \\
F_{u}(0, v)=\alpha_{1} g^{2}(v)+\alpha_{2} g^{4}(v), & F_{u u u}(0, v)=-\alpha_{1}^{3} g^{2}(v)-\alpha_{2}^{3} g^{4}(v) .
\end{array}
$$

Substituindo então essas expressões em (4.34), e pondo $g_{i j}(v)=\left\langle g^{i}(v), g^{j}(v)\right\rangle$, resulta

$$
\begin{gathered}
g_{11}+g_{33}+2 g_{13}=1, \\
\alpha_{1}^{2} g_{22}+\alpha_{2}^{2} g_{44}+2 \alpha_{1} \alpha_{2} g_{24}=\epsilon^{-1} B \sin ^{2} \theta, \\
\alpha_{1} g_{12}+\alpha_{2} g_{14}+\alpha_{1} g_{23}+\alpha_{2} g_{34}=0, \\
\alpha_{1}^{3} g_{12}+\alpha_{1} \alpha_{2}^{2} g_{23}+\alpha_{1}^{2} \alpha_{2} g_{14}+\alpha_{2}^{3} g_{34}=0, \\
\alpha_{1}^{4} g_{11}+\alpha_{2}^{4} g_{33}+2 \alpha_{1}^{2} \alpha_{2}^{2} g_{13}=D, \\
\alpha_{1}^{2} g_{11}+\alpha_{2}^{2} g_{33}+\left(\alpha_{1}^{2}+\alpha_{2}^{2}\right) g_{13}=\epsilon^{-2} \sin ^{2} \theta, \\
\alpha_{1}^{4} g_{22}+\alpha_{1}^{3} \alpha_{2} g_{24}+\alpha_{1} \alpha_{2}^{3} g_{24}+\alpha_{2}^{4} g_{44}=D, \\
\alpha_{1}^{5} g_{12}+\alpha_{1}^{3} \alpha_{2}^{2} g_{23}+\alpha_{1}^{2} \alpha_{2}^{3} g_{14}+\alpha_{2}^{5} g_{34}=0, \\
\alpha_{1}^{3} g_{12}+\alpha_{1}^{3} g_{23}+\alpha_{2}^{3} g_{14}+\alpha_{2}^{3} g_{34}=0, \\
\alpha_{1}^{6} g_{22}+\alpha_{2}^{6} g_{44}+2 \alpha_{1}^{3} \alpha_{2}^{3} g_{24}=E .
\end{gathered}
$$


De (4.37), 4.38), (4.42) e (4.43) segue que:

$$
g_{12}=g_{14}=g_{23}=g_{34}=0 .
$$

Temos ainda, de (4.35), 4.39) e 4.40), que

$$
g_{11}=\frac{\epsilon^{2}\left(D+\alpha_{2}^{4}\right)-2 B \sin ^{2} \theta \alpha_{2}^{2}}{\epsilon^{2}\left(\alpha_{1}^{2}-\alpha_{2}^{2}\right)^{2}}, \quad g_{13}=0, \quad g_{33}=\frac{\epsilon^{2}\left(D+\alpha_{1}^{4}\right)-2 B \sin ^{2} \theta \alpha_{1}^{2}}{\epsilon^{2}\left(\alpha_{1}^{2}-\alpha_{2}^{2}\right)^{2}} .
$$

Além disso, usando (4.36), (4.41) e (4.44), obtemos:

$$
g_{22}=\frac{\epsilon^{2}\left(E-2 D \alpha_{2}^{2}\right)+B \sin ^{2} \theta \alpha_{2}^{4}}{\epsilon^{2} \alpha_{1}^{2}\left(\alpha_{1}^{2}-\alpha_{2}^{2}\right)^{2}}, \quad g_{24}=0, \quad g_{44}=\frac{\epsilon^{2}\left(E-2 D \alpha_{1}^{2}\right)+B \sin ^{2} \theta \alpha_{1}^{4}}{\epsilon^{2} \alpha_{2}^{2}\left(\alpha_{1}^{2}-\alpha_{2}^{2}\right)^{2}} .
$$

Por fim, contas simples nos dão

$$
g_{11}=g_{22}=\frac{\epsilon}{2 B} \alpha_{2}, \quad g_{33}=g_{44}=\frac{\epsilon}{2 B} \alpha_{1} .
$$

\subsubsection{O resultado principal}

Para chegar ao resultado principal deste capítulo precisamos primeiramente relembrar que, olhando $\mathbb{S}_{\epsilon}^{3}$ em $\mathbb{R}^{4}$, seu grupo de isometrias pode ser identificado com o conjunto

$$
\left\{A \in O(4) ; A J_{1}= \pm J_{1} A\right\}
$$

onde $J_{1}$ é a estrutura complexa de $\mathbb{R}^{4}$ definida por

$$
J_{1}=\left(\begin{array}{cccc}
0 & -1 & 0 & 0 \\
1 & 0 & 0 & 0 \\
0 & 0 & 0 & -1 \\
0 & 0 & 1 & 0
\end{array}\right)
$$

e $O(4)$ é o grupo ortogonal. Suponhamos que seja dada uma família a 1-parâmetro $A(v), v \in(a, b) \subset \mathbb{R}$, consistindo de matrizes $4 \times 4$ ortogonais, que comutam com $J_{1}$. Para conseguirmos descrever explicitamente tal família, iremos usar outras duas estruturas complexas de $\mathbb{R}^{4}$ :

$$
J_{2}=\left(\begin{array}{cccc}
0 & 0 & 0 & -1 \\
0 & 0 & -1 & 0 \\
0 & 1 & 0 & 0 \\
1 & 0 & 0 & 0
\end{array}\right), \quad J_{3}=\left(\begin{array}{cccc}
0 & 0 & -1 & 0 \\
0 & 0 & 0 & 1 \\
1 & 0 & 0 & 0 \\
0 & -1 & 0 & 0
\end{array}\right)
$$


Observe que essas três estruturas satisfazem as relações:

$$
J_{1} J_{2}=-J_{3}, \quad J_{1} J_{3}=J_{2} \quad \text { e } \quad J_{2} J_{3}=-J_{1} .
$$

Agora, como $A(v)$ é ortogonal, se $r_{i}(v)$ denota a i-ésima linha da matriz $A(v)$ teremos que

$$
\left\|r_{i}(v)\right\|^{2}=1 \quad \text { e } \quad\left\langle r_{i}(v), r_{j}(v)\right\rangle=0 \quad \text { se } \quad i \neq j
$$

ou seja, as linhas da matriz $A(v)$ são vetores ortonormais de $\mathbb{R}^{4}$. Sendo assim, sem perda de generalidade, podemos tomar:

$$
\begin{aligned}
r_{1}(v)= & \left(\cos \xi_{1}(v) \cos \xi_{2}(v),-\cos \xi_{1}(v) \sin \xi_{2}(v),\right. \\
& \left.\sin \xi_{1}(v) \cos \xi_{3}(v),-\sin \xi_{1}(v) \cos \xi_{3}(v)\right),
\end{aligned}
$$

onde $\xi_{1}, \xi_{2}$ e $\xi_{3}$ são funções reais definidas em $(a, b)$. Como $A(v)$ comuta com $J_{1}$ devemos ter que $r_{2}(v)=J_{1} r_{1}(v)$. Além disso, que como os vetores $\left\{r_{1}, J_{1} r_{1}, J_{2} r_{1}, J_{3} r_{1}\right\}$ formam uma base ortonormal de $\mathbb{R}^{4}$, a terceira linha $r_{3}(v)$ é uma combinação linear deles. Sendo $r_{3}(v)$ um vetor unitário e ortogonal a $r_{1}(v)$ e a $r_{2}(v)$ temos:

$$
r_{3}(v)=\left\langle r_{3}(v), J_{2} r_{1}(v)\right\rangle J_{2} r_{1}(v)+\left\langle r_{3}(v), J_{3} r_{1}(v)\right\rangle J_{3} r_{1}(v)
$$

Logo, existe uma função $\xi \in C^{\infty}((a, b))$ tal que

$$
r_{3}(v)=\cos \xi(v) J_{2} r_{1}(v)+\sin \xi(v) J_{3} r_{1}(v)
$$

Notemos agora que, ainda por $A(v)$ comutar com $J_{1}$, obtemos que $r_{4}(v)=J_{1} r_{3}(v)$, assim

$$
r_{4}(v)=-\cos \xi(v) J_{3} r_{1}(v)+\sin \xi(v) J_{2} r_{1}(v)
$$

Portanto, qualquer família a 1-parâmetro $A(v)$ de matrizes ortogonais $4 \times 4$, que comutam com a estrutura complexa $J_{1}$, pode ser descrita por meio de quatro funções $\xi_{1}, \xi_{2}, \xi_{3}$ e $\xi$ como abaixo:

$$
A\left(\xi, \xi_{1}, \xi_{2}, \xi_{3}\right)(v)=\left(\begin{array}{c}
r_{1}(v) \\
J_{1} r_{1}(v) \\
\cos \xi(v) J_{2} r_{1}(v)+\sin \xi(v) J_{3} r_{1}(v) \\
-\cos \xi(v) J_{3} r_{1}(v)+\sin \xi(v) J_{2} r_{1}(v)
\end{array}\right)
$$


Teorema 4.2.5. Seja $M^{2}$ uma superfície na esfera de Berger $\mathbb{S}_{\epsilon}^{3}$ de ângulo constante $\theta \neq \pi / 2$. Então, localmente, o vetor posição de $M^{2}$ em $\mathbb{R}^{4}$ com respeito as coordenadas locais $(u, v)$ em $M$ definidas em (4.22) e (4.23) é dado por

$$
F(u, v)=A(v) \beta(u)
$$

onde

$$
\beta(u)=\left(\sqrt{g_{11}} \cos \left(\alpha_{1} u\right), \sqrt{g_{11}} \sin \left(\alpha_{1} u\right), \sqrt{g_{33}} \cos \left(\alpha_{2} u\right), \sqrt{g_{33}} \sin \left(\alpha_{2} u\right)\right),
$$

é uma geodésica no toro $\mathbb{S}^{1}\left(\sqrt{g_{11}}\right) \times \mathbb{S}^{1}\left(\sqrt{g_{33}}\right) \subset \mathbb{S}^{3}$, com $g_{11}, g_{33}, \alpha_{1}, \alpha_{2}$ sendo as quatro constantes dadas no Corolário 4.2 .4 e $A\left(\xi, \xi_{1}, \xi_{2}, \xi_{3}\right)(v)$ é uma família a 1-parâmetro de matrizes ortogonais $4 \times 4$ que comutam com a estrutura complexa $J_{1}$ (ver (4.46) com $\xi=$ constante, satisfazendo:

$$
\cos ^{2}\left(\xi_{1}(v)\right) \xi_{2}^{\prime}(v)-\sin ^{2}\left(\xi_{1}(v)\right) \xi_{3}^{\prime}(v)=0
$$

Reciprocamente, a parametrização $F(u, v)=A(v) \beta(u)$ com $A(v)$ e $\beta(u)$ como acima, define uma superfície na esfera de Berger $\mathbb{S}_{\epsilon}^{3}$ de ângulo constante $\theta \neq \pi / 2$.

Demonstração. Vimos anteriormente que, com respeito as coordenadas locais $(u, v)$ em $M$ definidas em (4.22) e (4.23), pelo Corolário 4.2.4, o vetor posição de $M$ em $\mathbb{R}^{4}$ é dado por:

$$
F(u, v)=\cos \left(\alpha_{1} u\right) g^{1}(v)+\sin \left(\alpha_{1} u\right) g^{2}(v)+\cos \left(\alpha_{2} u\right) g^{3}(v)+\sin \left(\alpha_{2} u\right) g^{4}(v),
$$

onde os campos vetoriais $\left\{g^{i}(v)\right\}_{i=1}^{4}$ são mutuamente ortogonais e satisfazem:

$$
\begin{aligned}
& \left\|g^{1}(v)\right\|=\left\|g^{2}(v)\right\|=\sqrt{g_{11}}=\text { constante } \\
& \left\|g^{3}(v)\right\|=\left\|g^{4}(v)\right\|=\sqrt{g_{33}}=\text { constante }
\end{aligned}
$$

Assim, definindo

$$
e_{i}(v)=\frac{g^{i}(v)}{\left\|g^{i}(v)\right\|}, \quad i \in\{1,2,3,4\},
$$

podemos reescrever $F$ como

$$
\begin{aligned}
F(u, v) & =\sqrt{g_{11}}\left(\cos \left(\alpha_{1} u\right) e_{1}(v)+\sin \left(\alpha_{1} u\right) e_{2}(v)\right) \\
& +\sqrt{g_{33}}\left(\cos \left(\alpha_{2} u\right) e_{3}(v)+\sin \left(\alpha_{2} u\right) e_{4}(v)\right) .
\end{aligned}
$$




\section{Observemos que}

$$
J_{1} F(u, v)=X_{1 \mid F(u, v)}=\epsilon E_{1 \mid F(u, v)}=\epsilon(T+\cos \theta N)_{\mid F(u, v)}=\epsilon\left(F_{u}+\cos \theta N\right)
$$

e

$$
\begin{aligned}
N_{\mid F(u, v)}= & \cos \theta E_{1 \mid F(u, v)}+\sin \theta \cos \varphi E_{2 \mid F(u, v)}+\sin \theta \sin \varphi E_{3 \mid F(u, v)} \\
= & \left(-\epsilon^{-1} \cos \theta F_{2}-\sin \theta \cos \varphi F_{4}-\sin \theta \sin \varphi F_{3},\right. \\
& \epsilon^{-1} \cos \theta F_{1}-\sin \theta \cos \varphi F_{3}+\sin \theta \sin \varphi F_{4}, \\
& -\epsilon^{-1} \cos \theta F_{4}+\sin \theta \cos \varphi F_{2}+\sin \theta \sin \varphi F_{1}, \\
& \left.\epsilon^{-1} \cos \theta F_{3}+\sin \theta \cos \varphi F_{1}-\sin \theta \sin \varphi F_{2}\right) .
\end{aligned}
$$

Logo, usando (4.29) e (4.34), obtemos as identidades:

$$
\begin{aligned}
& \left\langle J_{1} F, F_{u}\right\rangle=\epsilon^{-1} \sin ^{2} \theta, \\
& \left\langle J_{1} F, F_{u u}\right\rangle=0, \\
& \left\langle F_{u}, J_{1} F_{u u}\right\rangle=\epsilon^{-3} B \sin ^{2} \theta\left(\sin ^{2} \theta-2 B\right):=I, \\
& \left\langle J_{1} F_{u}, F_{\text {uuu }}\right\rangle=0, \\
& \left\langle J_{1} F_{u}, F_{\text {uu }}\right\rangle+\left\langle J_{1} F, F_{\text {uuи }}\right\rangle=0, \\
& \left\langle J_{1} F_{u u}, F_{u u u}\right\rangle+\left\langle J_{1} F_{u}, F_{\text {uиuи }}\right\rangle=0 .
\end{aligned}
$$

Avaliando agora a expressão de $F$ e de suas derivadas em $(0, v)$ temos:

$$
\begin{gathered}
F(0, v)=\sqrt{g_{11}} e_{1}(v)+\sqrt{g_{33}} e_{3}(v), \\
F_{u}(0, v)=\alpha_{1} \sqrt{g_{11}} e_{2}(v)+\alpha_{2} \sqrt{g_{33}} e_{4}(v), \\
F_{u u}(0, v)=-\alpha_{1}^{2} \sqrt{g_{11}} e_{1}(v)-\alpha_{2}^{2} \sqrt{g_{33}} e_{3}(v), \\
F_{\text {uuu }}(0, v)=-\alpha_{1}^{3} \sqrt{g_{11}} e_{2}(v)-\alpha_{2}^{3} \sqrt{g_{33}} e_{4}(v), \\
F_{\text {uuиu }}(0, v)=\alpha_{1}^{4} \sqrt{g_{11}} e_{1}(v)+\alpha_{2}^{4} \sqrt{g_{33}} e_{3}(v) .
\end{gathered}
$$


Assim, as expressões (4.50) tornam-se, respectivamente:

$$
\begin{gathered}
\alpha_{1} g_{11}\left\langle J_{1} e_{1}, e_{2}\right\rangle+\alpha_{2} g_{33}\left\langle J_{1} e_{3}, e_{4}\right\rangle \\
+\sqrt{g_{11} g_{33}}\left(\alpha_{1}\left\langle J_{1} e_{3}, e_{2}\right\rangle+\alpha_{2}\left\langle J_{1} e_{1}, e_{4}\right\rangle\right)=\epsilon^{-1} \sin ^{2} \theta, \\
\left\langle J_{1} e_{1}, e_{3}\right\rangle=0, \\
\alpha_{1}^{3} g_{11}\left\langle J_{1} e_{1}, e_{2}\right\rangle+\alpha_{2}^{3} g_{33}\left\langle J_{1} e_{3}, e_{4}\right\rangle \\
+\sqrt{g_{11} g_{33}}\left(\alpha_{1} \alpha_{2}^{2}\left\langle J_{1} e_{3}, e_{2}\right\rangle+\alpha_{1}^{2} \alpha_{2}\left\langle J_{1} e_{1}, e_{4}\right\rangle\right)=-I, \\
\left\langle J_{1} e_{2}, e_{4}\right\rangle=0, \\
\alpha_{1}\left\langle J_{1} e_{2}, e_{3}\right\rangle+\alpha_{2}\left\langle J_{1} e_{1}, e_{4}\right\rangle=0, \\
\alpha_{2}\left\langle J_{1} e_{2}, e_{3}\right\rangle+\alpha_{1}\left\langle J_{1} e_{1}, e_{4}\right\rangle=0 .
\end{gathered}
$$

Observemos que nas equações acima houve a divisão por

$$
\alpha_{1}^{2}-\alpha_{2}^{2}=4 \epsilon^{-1} \sqrt{B^{3} \cos ^{2} \theta}
$$

que é uma constante diferente de zero pois assumimos que $\theta \neq \pi / 2$. Assim das equações (4.55) e (4.56), e levando em consideração que $\alpha_{1}^{2}-\alpha_{2}^{2} \neq 0$, obtemos:

$$
\left\langle J_{1} e_{3}, e_{2}\right\rangle=0, \quad\left\langle J_{1} e_{1}, e_{4}\right\rangle=0 .
$$

Além disso,

$$
\left|\left\langle J_{1} e_{1}, e_{2}\right\rangle\right|=1=\left|\left\langle J_{1} e_{3}, e_{4}\right\rangle\right| .
$$

Substituindo agora (4.57) em (4.51) e (4.53), obtemos o sistema

$$
\left\{\begin{array}{l}
\alpha_{1} g_{11}\left\langle J_{1} e_{1}, e_{2}\right\rangle+\alpha_{2} g_{33}\left\langle J_{1} e_{3}, e_{4}\right\rangle=\epsilon^{-1} \sin ^{2} \theta \\
\alpha_{1}^{3} g_{11}\left\langle J_{1} e_{1}, e_{2}\right\rangle+\alpha_{2}^{3} g_{33}\left\langle J_{1} e_{3}, e_{4}\right\rangle=-I
\end{array}\right.
$$

o qual tem a solução dada por:

$$
\left\langle J_{1} e_{1}, e_{2}\right\rangle=\frac{\epsilon I+\alpha_{2}^{2} \sin ^{2} \theta}{\epsilon g_{11} \alpha_{1}\left(\alpha_{2}^{2}-\alpha_{1}^{2}\right)}, \quad\left\langle J_{1} e_{3}, e_{4}\right\rangle=-\frac{\epsilon I+\alpha_{1}^{2} \sin ^{2} \theta}{\epsilon g_{33} \alpha_{2}\left(\alpha_{2}^{2}-\alpha_{1}^{2}\right)} .
$$

Como

$$
g_{11} g_{33}=\frac{\sin ^{2} \theta}{4 B}, \quad \alpha_{1} \alpha_{2}=\frac{B}{\epsilon^{2}} \sin ^{2} \theta, \quad\left(\alpha_{1}^{2}-\alpha_{2}^{2}\right)^{2}=\frac{16 B^{3}}{\epsilon^{2}} \cos ^{2} \theta,
$$

segue que

$$
\left\langle J_{1} e_{1}, e_{2}\right\rangle\left\langle J_{1} e_{3}, e_{4}\right\rangle=-\frac{\left(\epsilon I+\alpha_{2}^{2} \sin ^{2} \theta\right)\left(\epsilon I+\alpha_{1}^{2} \sin ^{2} \theta\right)}{\epsilon^{2} g_{11} g_{33} \alpha_{1} \alpha_{2}\left(\alpha_{2}^{2}-\alpha_{1}^{2}\right)^{2}}=1
$$


Além disso, uma checagem direta mostra que $\left\langle J_{1} e_{1}, e_{2}\right\rangle>0$. Consequentemente,

$$
\left\langle J_{1} e_{1}, e_{2}\right\rangle=\left\langle J_{1} e_{3}, e_{4}\right\rangle=1 \text {. }
$$

Fixemos agora a base canônica de $\mathbb{R}^{4}$ dada por:

$$
\bar{e}_{1}=(1,0,0,0), \quad \bar{e}_{2}=(0,1,0,0), \quad \bar{e}_{3}=(0,0,1,0), \quad \bar{e}_{4}=(0,0,0,1) .
$$

Temos que, deverá existir uma família a 1-parâmetro de matrizes $4 \times 4$ ortogonais $A(v) \in O(4)$, com $J_{1} A(v)=A(v) J_{1}$, tal que:

$$
e_{i}(v)=A(v) \bar{e}_{i}, \quad i \in\{1,2,3,4\} .
$$

Substituindo (4.59) em 4.49) obtemos

$$
F(u, v)=A(v) \beta(u)
$$

onde

$$
\beta(u)=\left(\sqrt{g_{11}} \cos \left(\alpha_{1} u\right), \sqrt{g_{11}} \sin \left(\alpha_{1} u\right), \sqrt{g_{33}} \cos \left(\alpha_{2} u\right), \sqrt{g_{33}} \sin \left(\alpha_{2} u\right)\right)
$$

é uma geodésica do toro $\mathbb{S}^{1}\left(\sqrt{g_{11}}\right) \times \mathbb{S}^{1}\left(\sqrt{g_{33}}\right) \subset \mathbb{S}^{3}$. Vamos agora examinar a família a 1parâmetro $A(v)$ a qual, de acordo com (4.46), depende das quatro funções $\xi_{1}, \xi_{2}, \xi_{3}$ e $\xi$. Observe que, de (4.23) e levando em consideração (4.26), obtemos:

$$
\left\langle F_{v}, F_{v}\right\rangle=\sin ^{2} \theta=\text { constante. }
$$

Assim,

$$
\left\langle F_{u v}, F_{v}\right\rangle=0 \quad \text { e }\left\langle F_{u u v}, F_{v}\right\rangle+\left\langle F_{u v}, F_{u v}\right\rangle=0 .
$$

Denotando por $c_{1}, c_{2}, c_{3}$ e $c_{4}$ as quatro colunas da matriz $A(v)$, que é ortogonal e comuta com $J_{1}$, das equações (4.60) obtemos que:

$$
\left\{\begin{array}{l}
\left\langle c_{2}^{\prime}, c_{3}^{\prime}\right\rangle=0 \\
\left\langle c_{2}^{\prime}, c_{4}^{\prime}\right\rangle=0
\end{array}\right.
$$

onde com ' denotamos a derivada com respeito a $v$. Substituindo as expressões dos $c_{i}$ em função de $\xi_{1}, \xi_{2}, \xi_{3}$ e $\xi$ temos:

$$
c_{1}=\left(\begin{array}{c}
-\cos \xi_{1}(v) \sin \xi_{2}(v) \\
\cos \xi_{1}(v) \cos \xi_{2}(v) \\
-\cos \xi(v) \sin \xi_{1}(v) \cos \xi_{3}(v)-\sin \xi(v) \sin \xi_{1}(v) \sin \xi_{3}(v) \\
\cos \xi(v) \sin \xi_{1}(v) \sin \xi_{3}(v)-\sin \xi(v) \sin \xi_{1}(v) \cos \xi_{3}(v)
\end{array}\right)
$$




$$
\begin{gathered}
c_{2}=\left(\begin{array}{c}
-\cos \xi_{1}(v) \cos \xi_{2}(v) \\
-\cos \xi_{1}(v) \sin \xi_{2}(v) \\
-\cos \xi(v) \sin \xi_{1}(v) \sin \xi_{3}(v)+\sin \xi(v) \sin \xi_{1}(v) \cos \xi_{3}(v) \\
-\cos \xi(v) \sin \xi_{1}(v) \cos \xi_{3}(v)-\sin \xi(v) \sin \xi_{1}(v) \sin \xi_{3}(v)
\end{array}\right) \\
c_{3}=\left(\begin{array}{c}
\sin \xi_{1}(v) \cos \xi_{3}(v) \\
\sin \xi_{1}(v) \sin \xi_{3}(v) \\
-\cos \xi(v) \cos \xi_{1}(v) \sin \xi_{2}(v)+\sin \xi(v) \cos \xi_{1}(v) \cos \xi_{2}(v) \\
-\cos \xi(v) \cos \xi_{1}(v) \cos \xi_{2}(v)-\sin \xi(v) \cos \xi_{1}(v) \sin \xi_{2}(v)
\end{array}\right) \\
c_{4}=\left(\begin{array}{c}
-\sin \xi_{1}(v) \sin \xi_{3}(v) \\
\sin \xi_{1}(v) \cos \xi_{3}(v) \\
\cos \xi(v) \cos \xi_{1}(v) \cos \xi_{2}(v)+\sin \xi(v) \cos \xi_{1}(v) \sin \xi_{2}(v) \\
-\cos \xi(v) \cos \xi_{1}(v) \sin \xi_{2}(v)+\sin \xi(v) \cos \xi_{1}(v) \cos \xi_{2}(v)
\end{array}\right)
\end{gathered}
$$

Portanto a equação (4.61) se torna:

$$
\left\{\begin{array}{l}
\left\langle c_{2}^{\prime}, c_{4}^{\prime}\right\rangle=\frac{1}{2} \xi^{\prime}\left(-2 \sin \left(\xi_{2}-\xi_{3}\right) \xi_{1}^{\prime}+\cos \left(\xi_{2}-\xi_{3}\right) \sin \left(2 \xi_{1}\right) \sin \left(-\xi^{\prime}+\xi_{2}^{\prime}+\xi_{3}^{\prime}\right)\right)=0 \\
\left\langle c_{2}^{\prime}, c_{3}^{\prime}\right\rangle=\frac{1}{2} \xi^{\prime}\left(-2 \sin \left(\xi_{2}-\xi_{3}\right) \xi_{1}^{\prime}+\sin \left(\xi_{2}-\xi_{3}\right) \sin \left(2 \xi_{1}\right) \sin \left(\xi^{\prime}-\xi_{2}^{\prime}-\xi_{3}^{\prime}\right)\right)=0 .
\end{array}\right.
$$

Pondo

$$
2 \frac{\left\langle c_{2}^{\prime}, c_{4}^{\prime}\right\rangle}{\xi^{\prime}}=h(v) \quad \text { e } \quad 2 \frac{\left\langle c_{2}^{\prime}, c_{3}^{\prime}\right\rangle}{\xi^{\prime}}=k(v)
$$

temos

$$
\left\{\begin{array}{l}
\xi^{\prime} h(v)=0, \\
\xi^{\prime} k(v)=0,
\end{array}\right.
$$

e as funções $h(v), k(v)$ satisfazem a relação:

$$
h^{2}+k^{2}=4\left(\xi_{1}^{\prime}\right)^{2}+\sin ^{2}\left(2 \xi_{1}\right)\left(-\xi^{\prime}+\xi_{2}^{\prime}+\xi_{3}^{\prime 2}\right)^{2} .
$$

Assim de (4.62) segue que podem ocorrer somente duas possibilidades:

1. $\xi^{\prime}=0$, ou seja, $\xi=$ constante;

2. $4\left(\xi_{1}^{\prime}\right)^{2}+\sin ^{2}\left(2 \xi_{1}\right)\left(-\xi^{\prime}+\xi_{2}^{\prime}+\xi_{3}^{\prime 2}\right)^{2}=0$. 
Mostraremos que o segundo caso não pode ocorrer. Mais precisamente, mostraremos que se o caso 2. ocorrer, então $F(u, v)=A(v) \beta(u)$ é um tubo de Hopf, isto é, o campo vetorial de Hopf $E_{1}$ será tangente à superfície em todo ponto. Para este fim calcularemos o campo normal unitário $N$ a partir da parametrização $F(u, v)=A(v) \beta(u)$. Escrevendo

$$
F_{u}=g_{\epsilon}\left(F_{u}, E_{1}\right) E_{1}+g_{\epsilon}\left(F_{u}, E_{2}\right) E_{2}+g_{\epsilon}\left(F_{u}, E_{3}\right) E_{3}
$$

$\mathrm{e}$

$$
F_{v}=g_{\epsilon}\left(F_{v}, E_{1}\right) E_{1}+g_{\epsilon}\left(F_{v}, E_{2}\right) E_{2}+g_{\epsilon}\left(F_{v}, E_{3}\right) E_{3},
$$

onde $\left\{E_{1}, E_{2}, E_{3}\right\}$ é a base do espaço tangente a $\mathbb{S}_{\epsilon}^{3}$ definida em (4.2), resulta:

$$
N=\frac{N_{1} E_{1}+N_{2} E_{2}+N_{3} E_{3}}{\sqrt{N_{1}^{2}+N_{2}^{2}+N_{3}^{2}}},
$$

onde

$$
\left\{\begin{array}{l}
N_{1}=g_{\epsilon}\left(F_{u}, E_{2}\right) g_{\epsilon}\left(F_{v}, E_{3}\right)-g_{\epsilon}\left(F_{u}, E_{3}\right) g_{\epsilon}\left(F_{v}, E_{2}\right), \\
N_{2}=g_{\epsilon}\left(F_{u}, E_{3}\right) g_{\epsilon}\left(F_{v}, E_{1}\right)-g_{\epsilon}\left(F_{u}, E_{1}\right) g_{\epsilon}\left(F_{v}, E_{3}\right), \\
N_{3}=g_{\epsilon}\left(F_{u}, E_{1}\right) g_{\epsilon}\left(F_{v}, E_{2}\right)-g_{\epsilon}\left(F_{u}, E_{2}\right) g_{\epsilon}\left(F_{v}, E_{1}\right) .
\end{array}\right.
$$

A partir de cálculos simples, porém longos (os quais podem ser feitos usando o software Mathematica), temos que

$$
\begin{aligned}
N_{1} & =\frac{1}{2}\left(\alpha_{1}-\alpha_{2}\right) \sqrt{g_{11}} \sqrt{g_{33}}\left(2 \cos \left(\alpha_{1} u-\alpha_{2} u+\xi_{2}-\xi_{3}\right) \xi_{1}^{\prime}\right. \\
& \left.+\sin \left(2 \xi_{1}\right) \sin \left(\alpha_{1} u-\alpha_{2} u+\xi_{2}-\xi_{3}\right)\right)\left(-\xi^{\prime}+\xi_{2}^{\prime}+\xi_{3}^{\prime}\right) .
\end{aligned}
$$

Observe que o caso 2, ocorre se, e somente se, ou

$$
\xi_{1}=\text { constante }=\frac{k \pi}{2}, \quad k \in \mathbb{Z},
$$

ou

$$
\xi_{1}=\text { constante } \neq \frac{k \pi}{2}, k \in \mathbb{Z} \quad \text { e } \quad-\xi^{\prime}+\xi_{2}^{\prime}+\xi_{3}^{\prime}=0 .
$$

Em ambos os casos obtemos $N_{1}=0$, o que implica que

$$
g_{\epsilon}\left(N, J_{1} F\right)=\epsilon g_{\epsilon}\left(N, E_{1}\right)=0,
$$

ou seja, o campo vetorial de Hopf é tangente à superfície, o que não pode ocorrer pois estamos sob a hipótese que $\theta \neq \pi / 2$. Assim provamos que $\xi=$ constante. Usando agora (4.23) e calculando os campos $E_{i}$ em $F=\left(F_{1}, F_{2}, F_{3}, F_{4}\right)$ obtemos:

$$
g_{\epsilon}\left(F_{v}, \epsilon^{-1} J_{1} F\right)=a \sin ^{2} \theta \quad \text { e } \quad g_{\epsilon}\left(F_{v}, F_{u}\right)=a \sin ^{2} \theta .
$$


Assim,

$$
g_{\epsilon}\left(F_{v}, J_{1} F\right)-\epsilon g_{\epsilon}\left(F_{v}, F_{u}\right)=0 .
$$

Usando novamente o software Mathematica, podemos facilmente calcular (4.64) quando

$$
F(u, v)=A(v) \beta(u)
$$

e achamos,

$$
\begin{aligned}
0 & =g_{\epsilon}\left(F_{v}, J_{1} F\right)-\epsilon g_{\epsilon}\left(F_{v}, F_{u}\right) \\
& =-\epsilon \cos \theta \sqrt{B}\left[\cos ^{2} \theta\left(\xi_{1}(v)\right) \xi_{2}^{\prime}(v)-\sin ^{2}\left(\xi_{1}(v)\right) \xi_{3}^{\prime}(v)\right] .
\end{aligned}
$$

Como $\theta \neq \pi / 2$, temos que:

$$
\cos ^{2} \theta\left(\xi_{1}(v)\right) \xi_{2}^{\prime}(v)-\sin ^{2}\left(\xi_{1}(v)\right) \xi_{3}^{\prime}(v)=0
$$

A recíproca do teorema pode ser provada da seguinte maneira: seja $F(u, v)=A(v) \beta(u)$ uma parametrização de uma superfície em $\mathbb{S}_{\epsilon}^{3} \operatorname{com} \beta(u)$ dada como em (4.47), $A(v)=A\left(\xi, \xi_{1}, \xi_{2}, \xi_{3}\right)(v)$ $\operatorname{com} \xi=$ constante e $\xi_{1}, \xi_{2}, \xi_{3}$ satisfazendo (4.48). Então, escrevendo

$$
\alpha_{1}=\frac{2 B}{\epsilon} g_{33}, \quad \alpha_{2}=\frac{2 B}{\epsilon} g_{11} \quad \text { e } \quad g_{11}=1-g_{33}
$$

temos que, as componentes de $N$ podem ser escritas da seguinte maneira:

$$
\left\{\begin{array}{l}
N_{1}=\frac{1}{\epsilon} B \sqrt{\left(1-g_{33}\right)} g_{33}\left(2 g_{33}-1\right) \zeta, \\
N_{2}=2 B\left(g_{33}-1\right) g_{33} \sin \left[\frac{(2 B u)}{\epsilon}+\alpha\right] \zeta, \\
N_{3}=2 B\left(g_{33}-1\right) g_{33} \cos \left[\frac{(2 B u)}{\epsilon}+\alpha\right] \zeta,
\end{array}\right.
$$

onde

$$
\begin{aligned}
\zeta & =2 \xi_{1}^{\prime}(v) \cos \left(\frac{2 B\left(1-2 g_{33}\right) u}{\epsilon}-\xi_{2}+\xi_{3}\right) \\
& -\sin \left(2 \xi_{1}\right)\left(\xi_{2}^{\prime}+\xi_{3}^{\prime}\right) \sin \left(\frac{2 B\left(1-2 g_{33}\right) u}{\epsilon}-\xi_{2}+\xi_{3}\right) .
\end{aligned}
$$

Substituindo os valores de $B$ e de $g_{33}$, obtemos:

$$
\frac{g_{\epsilon}\left(N, E_{1}\right)^{2}}{g_{\epsilon}(N, N)}=\frac{N_{2}^{2}}{N_{1}^{2}+N_{2}^{2}+N_{3}^{2}}=\cos ^{2} \theta,
$$

o que implica que $F(u, v)$ define uma superfície de ângulo constante $\theta$ em $\mathbb{S}_{\epsilon}^{3}$. 
Exemplo 4.2.6. Vamos agora achar um exemplo explícito de uma família a 1-parâmetro $A(v)$ como no Teorema 4.2.5, Como $\xi=$ constante e $\xi_{1}(v), \xi_{2}(v), \xi_{3}(v)$ são soluções de (4.48) podemos tomar

$$
\xi=\frac{\pi}{2}, \quad \xi_{1}=\frac{\pi}{4}, \quad \xi_{2}(v)=\xi_{3}(v)
$$

Assim $A(v)$ se torna:

$$
A(v)=\frac{1}{\sqrt{2}}\left(\begin{array}{cccc}
\cos \xi_{2}(v) & -\sin \xi_{2}(v) & \cos \xi_{2}(v) & -\sin \xi_{2}(v) \\
\sin \xi_{2}(v) & \cos \xi_{2}(v) & \sin \xi_{2}(v) & \cos \xi_{2}(v) \\
-\cos \xi_{2}(v) & -\sin \xi_{2}(v) & \cos \xi_{2}(v) & \sin \xi_{2}(v) \\
\sin \xi_{2}(v) & -\cos \xi_{2}(v) & -\sin \xi_{2}(v) & \cos \xi_{2}(v)
\end{array}\right)
$$

Usando a notação do Teorema 4.2.5, a aplicação

$$
F(u, v)=A(v) \beta(u)
$$

nos dá uma imersão explícita de uma superfície de ângulo constante na esfera de Berger.

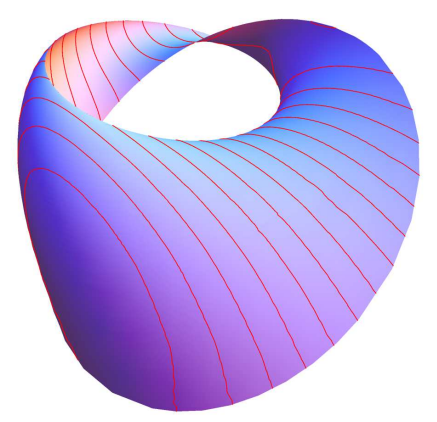

Figura 4.1: Projeção estereográfica em $\mathbb{R}^{3}$ da superfície de ângulo constante $\pi / 4$ em $\mathbb{S}^{3}$ dada por $F$ no exemplo acima. 



\begin{tabular}{l|l|}
\hline APÊNDICE \\
\hline \\
4 \\
\hline
\end{tabular}

\section{Imersões isométricas e campos de}

Killing

Neste apêndice vamos relembrar as equações básicas de uma imersão isométrica entre duas variedades Riemannianas, que foram usadas ao longo desta dissertação. Inicialmente, obteremos as fórmulas de Gauss e de Weingarten e, baseadas nelas, daremos as equações de Gauss e de Weingarten. Também daremos a definição de campo de Killing de uma variedade Riemanniana e como podemos relacioná-lo com o grupo de isometrias da variedade.

O conteúdo deste apêndice será exposto de forma sucinta, mais detalhes sobre esse assunto podem ser encontrados em [15], [10] e [21]. 


\section{A.1 Equações fundamentais de uma imersão isométrica}

Definição A.1.1. Sejam $\left(M^{m},\langle,\rangle_{M}\right)$ e $\left(N^{n},\langle,\rangle_{N}\right)$ duas variedades Riemannianas de dimensão $m$ e $n$, respectivamente. Dizemos que uma aplicação diferenciável $f: M^{m} \longrightarrow N^{n}$ é uma imersão isométrica se ela for uma imersão e, para todo ponto $p \in M$, resulta

$$
\langle u, v\rangle_{M}=\left\langle d f_{p}(u), d f_{p}(v)\right\rangle_{N}, \quad u, v \in T_{p} M,
$$

onde $d f_{p}: T_{p} M \longrightarrow T_{f(p)} N$ é a diferencial de $f$ no ponto $p$. O número $k=(n-m)$ é chamado de codimensão de $f$.

Seja $f: M^{m} \longrightarrow N^{n}$ uma imersão isométrica. Para cada $p \in M$ existe uma vizinhança $U$ em $M$ tal que, a restrição de $f$ a $U$ é um mergulho, assim o conjunto $f(U) \subset N$ é uma subvariedade Riemanniana $m$-dimensional de $N$. Além disso, podemos identificar $U$ com sua imagem pela $f$, ou seja, considerar $f$ como sendo, localmente, a aplicação inclusão. Desta forma, podemos olhar o espaço $T_{p} M$, tangente à variedade $M$ no ponto $p$, como sendo um subespaço vetorial do espaço $T_{p} N$, tangente a $N$ no ponto $p$. Assim, o produto interno em $T_{p} N$ decompõe $T_{p} N$ na soma direta

$$
T_{p} N=T_{p} M \oplus\left(T_{p} M\right)^{\perp},
$$

onde $\left(T_{p} M\right)^{\perp}$ é o complemento ortogonal de $T_{p} M$ em $T_{p} N$.

Portanto, se $\bar{\nabla}$ representa a conexão de Levi-Civita de $N$, dados $X, Y \in \chi(M)$, temos a decomposição

$$
\bar{\nabla}_{X} Y=\left(\bar{\nabla}_{X} Y\right)^{\top}+\left(\bar{\nabla}_{X} Y\right)^{\perp}
$$

Observe que, escrevendo

$$
\nabla_{X} Y=\left(\bar{\nabla}_{X} Y\right)^{\top}, \quad X, Y \in \chi(M),
$$

pela unicidade da conexão de Riemanniana, teremos que $\nabla$ é exatamente a conexão de Levi-Civita da variedade $M$. A partir daqui, obtemos a seguinte equação:

$$
\bar{\nabla}_{X} Y=\nabla_{X} Y+h(X, Y)
$$

que é chamada de fórmula de Gauss e define uma aplicação

$$
h: T M \times T M \longrightarrow(T M)^{\perp}
$$


chamada segunda forma fundamental de $f$. Segue diretamente das propriedades das conexões de Levi-Civita, $\bar{\nabla}$ e $\nabla$, que $h$ é bilinear e simétrica sobre o anel $C^{\infty}(M)$ das funções diferenciáveis de $M$.

Considere, agora, os campos vetoriais $X \in T M$ e $\xi \in(T M)^{\perp}$ e defina

$$
A_{\xi}(X):=-\left(\bar{\nabla}_{X} \xi\right)^{\top}
$$

Note que, para cada $Y \in T M$, resulta

$$
0=X\langle\xi, Y\rangle=\left\langle\bar{\nabla}_{X} \xi, Y\right\rangle+\left\langle\xi, \bar{\nabla}_{X} Y\right\rangle
$$

assim, a fórmula (A.1) nos dá

$$
\left\langle A_{\xi}(X), Y\right\rangle=\langle h(X, Y), \xi\rangle
$$

Isso implica que, a aplicação

$$
A: T M \times(T M)^{\perp} \longrightarrow T M
$$

dada por $A(X, \xi)=A_{\xi}(X)$ é bilinear sobre $C^{\infty}(M)$ e a aplicação $A_{\xi}: T M \longrightarrow T M$ é linear sobre $C^{\infty}(M)$. Além disso, como $h$ é simétrica, segue que $A_{\xi}$ é um operador auto-adjunto o qual é chamado de operador forma. A equação

$$
\bar{\nabla}_{X} \xi=-A_{\xi}(X)+\left(\bar{\nabla}_{X} \xi\right)^{\perp}
$$

é chamada de fórmula de Weingarten.

Observe que, se a codimensão de $f$ é 1 , dados $X \in T M$ e $\xi \in(T M)^{\perp}$ a componente do campo $\bar{\nabla}_{X} \xi$ que é normal a $M$ é nula, logo a formula de Weingarten poderá ser reescrita da forma:

$$
\bar{\nabla}_{X} \xi=-A_{\xi}(X)
$$

Como, neste caso, $(T M)^{\perp}$ tem dimensão 1 , podemos supor que $\xi$ é o gerador unitário de $(T M)^{\perp}$ e escreveremos (por simplicidade) $A_{\xi}=A$.

Definição A.1.2. Seja $\left(M^{m},\langle\rangle,\right)$ uma variedade Riemanniana. A curvatura $R$ de $M$ é uma correspondência que associa a cada par $X, Y \in \chi(M)$ a aplicação

$$
R(X, Y): \chi(M) \longrightarrow \chi(M)
$$


dada por

$$
R(X, Y) Z=\nabla_{X} \nabla_{Y} Z-\nabla_{Y} \nabla_{X} Z-\nabla_{[X, Y]} Z
$$

onde $\nabla$ é a conexão de Levi-Civita de $M$.

Dado um ponto $p \in M$ e um subespaço bidimensional $\sigma \subset T_{p} M$, chamamos de curvatura seccional de $\sigma$ em $p$ o número real

$$
K(\sigma)=K(x, y)=\frac{\langle R(x, y) y, x\rangle}{\sqrt{\|x\|^{2}\|y\|^{2}-\langle x, y\rangle^{2}}},
$$

onde $\{x, y\}$ é uma base qualquer de $\sigma$.

Proposição A.1.3. A curvatura $R$ de uma variedade Riemanniana $(M,\langle\rangle$,$) é multilinear em$ $\chi(M) \times \chi(M) \times \chi(M)$, isto é,

$$
\begin{gathered}
R(f X+Y, Z) W=f R(X, Z) W+R(Y, Z) W, \\
R(X, f Y+Z) W=f R(X, Y) W+R(X, Z) W, \\
R(X, Y)(f Z+W)=f R(X, Y) Z+R(X, Y) W,
\end{gathered}
$$

onde $f \in C^{\infty}(M)$ e $X, Y, Z, W \in \chi(M)$. Além disso, ela satisfaz as seguintes propriedades:

1. $R(X, Y) Z+R(Y, Z) X+R(Z, X) Y=0$;

2. $\langle R(X, Y) Z, W\rangle=-\langle R(Y, X) Z, W\rangle$;

3. $\langle R(X, Y) Z, W\rangle=-\langle R(X, Y) W, Z\rangle$.

Demonstração. A demonstração, que segue das propriedades da conexão Riemanniana, pode ser encontrada em [15], por exemplo.

Seja $f: M^{m} \longrightarrow N^{n}$ uma imersão isométrica entre duas variedades Riemannianas $M^{m}$ e $N^{n}$, cujas curvaturas sejam dadas por $R$ e $\widetilde{R}$, respectivamente. Dados $X, Y, Z, W \in T M$, a partir das fórmulas de Gauss e de Weingarten obtemos a equação

$$
\begin{aligned}
\langle R(X, Y) Z, W\rangle & =\langle\widetilde{R}(X, Y) Z, W\rangle+\langle h(X, W), h(Y, Z)\rangle \\
& -\langle h(X, Z), h(Y, W)\rangle
\end{aligned}
$$


chamada equação de Gauss, e a equação

$$
(\widetilde{R}(X, Y) Z)^{\perp}=\left(\nabla_{X}^{\perp} h\right)(Y, Z)-\left(\nabla_{Y}^{\perp} h\right)(X, Z)
$$

denominada equação de Codazzi, onde

$$
\left(\nabla_{X}^{\perp} h\right)(Y, Z)=\left(\bar{\nabla}_{X} h(Y, Z)\right)^{\perp}-h\left(\nabla_{X} Y, Z\right)-h\left(Y, \nabla_{X} Z\right) .
$$

Quando a codimensão da imersão é 1, segue da equação (A.4) que as equações de Gauss e de Codazzi podem ser reescritas como

$$
\begin{aligned}
\langle R(X, Y) Z, W\rangle & =\langle\widetilde{R}(X, Y) Z, W\rangle+\langle A(Y), Z\rangle\langle A(X), W\rangle \\
& -\langle A(X), Z\rangle\langle A(Y), W\rangle
\end{aligned}
$$

$\mathrm{e}$

$$
\widetilde{R}(X, Y) N=\nabla_{Y} A(X)-\nabla_{X} A(Y)+A([X, Y])
$$

respectivamente, onde $N \in(T M)^{\perp}$.

\section{A.2 Campos de Killing}

Nesta seção, iremos primeiramente lembrar as noções de curva integral e de fluxo local de um campo em uma variedade diferenciável.

Definição A.2.1. Seja $M$ uma variedade diferenciável e $X \in \chi(M)$. Uma curva diferenciável $\alpha: I \subset \mathbb{R} \longrightarrow M$ é chamada uma curva integral de $X$ se

$$
\alpha^{\prime}(t)=X(\alpha(t)), \quad t \in I
$$

Definição A.2.2. Sejam $M$ uma variedade diferenciável e $X \in \chi(M)$. Um fluxo local para o campo $X$ em torno de um ponto $q \in M$ é uma aplicação $\varphi:(-\epsilon, \epsilon) \times U \longrightarrow M$ de classe $C^{\infty}$, onde $U \subset M$ é um aberto contendo $q$, que satisfaz as seguintes propriedades:

(a) para cada $p \in U$, a curva $\alpha_{p}:(-\epsilon, \epsilon) \longrightarrow M$, dada por $\alpha_{p}(t)=\varphi(t, p)$, é uma curva integral de $X, \operatorname{com} \alpha_{p}(0)=p$ 
(b) para cada $t \in(-\epsilon, \epsilon)$, a aplicação $\varphi_{t}: U \longrightarrow M$, dada por $\varphi_{t}(p)=\varphi(t, p)$, é um difeomorfismo sobre sua imagem.

Observação A.2.3. Sejam $X \in \chi(M)$ e $\varphi:(-\epsilon, \epsilon) \times U \longrightarrow M$ um fluxo local para $X$. Então, para cada $p \in U$, desde que ambos os lados estejam definidos, $\varphi$ satisfaz:

$$
\varphi_{s} \circ \varphi_{t}=\varphi_{s+t}, \quad t, s, s+t \in(-\epsilon, \epsilon)
$$

Esta propriedade é chamada de propriedade local de grupo e a família $\left(\varphi_{t}\right)_{t \in(-\epsilon, \epsilon)}$ é chamada de subgrupo local de difeomorfismos locais de $M$.

Teorema A.2.4. Sejam $M$ uma variedade diferenciável e $X \in \chi(M)$. Dado um ponto $q \in M$, existe um fluxo local $\varphi:(-\epsilon, \epsilon) \times U \longrightarrow M$ para $X$ em torno de q tal que, para cada $p \in U$ a curva $\alpha_{p}:(-\epsilon, \epsilon) \longrightarrow M$, dada por $\alpha_{p}(t)=\varphi(t, p)$, é a única curva integral de $X$, com $\varphi(0, p)=p$.

Demonstração. Ver [6], pág. 132.

Definição A.2.5. Seja $\varphi: M \longrightarrow N$ uma aplicação diferenciável. Se $\omega$ é uma $r$-forma $\operatorname{com} r \geq 1$, definimos

$$
\left(\varphi^{*} \omega\right)\left(v_{1}, \ldots, v_{r}\right)=\omega\left(d \varphi v_{1}, \ldots, d \varphi v_{r}\right),
$$

para todos $v_{i} \in T_{p} M, p \in M$. Esta aplicação é chamada de pullback de $\omega$ por $\varphi$.

Definição A.2.6. Sejam $X \in \chi(M),\left(\varphi_{t}\right)_{t \in(-\epsilon, \epsilon)}$ o subgrupo local de difeomorfismos locais gerado por $X$ e $S$ um tensor em $M$. A derivada de Lie de $S$ na direção de $X$ é definida como

$$
L_{X} S:=\lim _{t \rightarrow 0} \frac{1}{t}\left(\varphi_{t}^{*}(S)-S\right)
$$

Teorema A.2.7. Seja $(M, g)$ uma variedade Riemanniana e $X \in \chi(M)$. Temos

1. Se $f: M \longrightarrow \mathbb{R}$ é uma função diferenciável, então

$$
L_{X}(f)=d f(X)=X(f)
$$

2. Se $Y \in \chi(M)$, então

$$
L_{X} Y=[X, Y]
$$


3. Se wé uma r-forma em $M$, dados $X_{1}, \ldots, X_{r} \in \chi(M)$ temos

$$
L_{X} \omega\left(X_{1}, \ldots, X_{r}\right)=X\left(\omega\left(X_{1}, \ldots, X_{r}\right)\right)+\sum_{i=1}^{r} \omega\left(X_{1}, \ldots,\left[X_{1}, X_{i}\right], \ldots, X_{r}\right) .
$$

Demonstração. Ver [21], página 51.

Observemos que, se no item 3 , do teorema anterior, $\omega$ for o tensor métrico $g$, então a equação A.13 poderá ser reescrita na forma

$$
L_{X} g(Y, Z)=X(g(Y, Z))-g([X, Y], Z)-g(Y,[X, Z]), \quad X, Y, Z \in \chi(M)
$$

Usando, então, a propriedade de simetria da conexão de Levi-Civita, temos:

$$
L_{X} g(Y, Z)=g\left(\nabla_{Y} X, Z\right)+g\left(Y, \nabla_{Z} X\right) \quad X, Y, Z \in \chi(M),
$$

onde $\nabla$ é a conexão Riemanniana da variedade $M$.

Definição A.2.8. Seja $(M, g)$ uma variedade Riemanniana. Dizemos que um campo $V \in \chi(M)$ é um campo de Killing ou isometria infinitesimal em $M$ se

$$
L_{V} g=0 .
$$

Segue da definição acima e da equação A.14 que um campo $V \in \chi(M)$ é um campo de Killing em $M$ se, e somente se, ele satisfaz a equação

$$
g\left(\nabla_{X} V, Y\right)+g\left(X, \nabla_{Y} V\right)=0
$$

a qual é chamada de equação de Killing.

O conjunto das isometrias de uma variedade Riemanniana $(M, g)$ forma um subgrupo do grupo dos difeomorfismos de $M$. Assim, se um campo gera uma família a um parâmetro constituída por isometrias, dizemos que ele gera um subgrupo a um parâmetro de isometrias. Veremos agora que a noção de isometria está intimamente ligada aos campos de Killing.

Proposição A.2.9. Sejam $(M, g)$ uma variedade Riemanniana e $V \in \chi(M)$. Então $V$ é um campo de Killing se, e somente se, ele gera um subgrupo a um parâmetro de isometrias. 
Demonstração. Se cada $\varphi_{t}$ é uma isometria, então $\varphi_{t}^{*}(g)=g$. Assim $L_{V} g=0$. Reciprocamente, se $L_{V} g=0$, seja $\left(\varphi_{t}\right)_{t \in(-\epsilon, \epsilon)}$ o fluxo local de $V$. Se $v$ é um vetor tangente em um ponto no domínio do fluxo, então $w=d \varphi_{s}(v)$ para $s$ suficientemente pequeno. Logo

$$
\lim _{t \rightarrow 0} \frac{1}{t}\left(g\left(d \varphi_{t}(w), d \varphi_{t}(w)\right)-g(w, w)\right)=0 .
$$

Como

$$
\varphi_{s} \circ \varphi_{t}=\varphi_{s+t}
$$

temos que

$$
\lim _{t \rightarrow 0} \frac{1}{t}\left(g\left(d \varphi_{s+t}(v), d \varphi_{s+t}\right)-g\left(d \varphi_{s}(v), d \varphi_{s}(v)\right)\right)=0
$$

Isto mostra que a função a valores reais $s \longmapsto g\left(d \varphi_{s}(v), d \varphi_{s}(v)\right)$ tem derivada identicamente nula, então a mesma é constante. Assim

$$
g\left(d \varphi_{s}(v), d \varphi_{s}(v)\right)=g(v, v)
$$

para todo $v$ e $s$.

Em seguida iremos determinar uma base de campos de Killing em $\mathbb{R}^{3}$.

Teorema A.2.10. Seja $V \in \chi\left(\mathbb{R}^{3}\right)$. Então $V$ é um campo de Killing se, e somente se, ele é da forma:

$$
V(x, y, z)=(a y+b z+c) \partial_{x}+(-a x-d z+e) \partial_{y}+(d y-b x+f) \partial_{z},
$$

onde $a, b, c, d, e, f \in \mathbb{R}$.

Demonstração. Considere um campo em $\mathbb{R}^{3}$, escrito como $V=\sum_{i=1}^{3} a_{i} \partial_{x_{i}}$, onde $a_{i} \in C^{\infty}\left(\mathbb{R}^{3}\right)$. Então $V$ é um campo de Killing se, e somente se,

$$
\left\langle\nabla_{\partial_{x_{i}}} V, \partial_{x_{j}}\right\rangle+\left\langle\nabla_{\partial_{x_{j}}} V, \partial_{x_{i}}\right\rangle=0
$$

para todo $i, j=1,2,3$. Como

$$
\left\langle\nabla_{\partial_{x_{i}}} V, \partial_{x_{i}}\right\rangle=\partial_{x_{i}}\left(a_{i}\right) \text { e }\left\langle\nabla_{\partial_{x_{i}}} V, \partial_{x_{j}}\right\rangle=\partial_{x_{i}}\left(a_{j}\right)
$$


a equação (A.18) é satisfeita se, e somente se,

$$
\left\{\begin{array}{l}
\partial_{x_{i}}\left(a_{i}\right)=0 \\
\partial_{x_{i}}\left(a_{j}\right)=-\partial_{x_{j}}\left(a_{i}\right),
\end{array}\right.
$$

para todo $i, j$. Assim, indicando por $a_{1}(x, y, z)=a(x, y, z), a_{2}(x, y, z)=b(x, y, z), a_{3}(x, y, z)=$ $c(x, y, z)$, o sistema (A.19) se torna:

$$
\begin{aligned}
& a_{x}(x, y, z)=b_{y}(x, y, z)=c_{z}(x, y, z)=0 \\
& a_{y}(x, y, z)=-b_{x}(x, y, z) \\
& a_{z}(x, y, z)=-c_{x}(x, y, z) \\
& b_{z}(x, y, z)=-c_{y}(x, y, z) .
\end{aligned}
$$

De A.20a temos que $a=a(y, z), b=b(x, z), c=c(x, y)$ e, também, existem funções $f, g, h, k$, $n, w, m, l, p, r \in C^{\infty}(\mathbb{R})$ tais que:

$$
\begin{aligned}
& a(y, z)=f(z) y+g(z), \\
& a(y, z)=h(y) z+k(y), \\
& b(x, z)=m(z) x+l(z), \\
& c(x, y)=n(x) y+w(x), \\
& c(x, y)=p(y) x+r(y) .
\end{aligned}
$$

De (A.20b) segue que $m(z)=-f(z)$, logo podemos escrever

$$
b(x, z)=-f(z) x+l(z) .
$$

De A.20c temos que $p(y)=-h(y)$, assim

$$
c(x, y)=-h(y) x+r(y) .
$$

Por outro lado, ainda de (A.20c), resulta que

$$
f^{\prime}(z) y+g^{\prime}(z)=-n^{\prime}(x) y-w^{\prime}(x)
$$

$\log 0$

$$
f^{\prime}(z)=-n^{\prime}(x)=c_{1} \quad \text { e } \quad g^{\prime}(z)=-w^{\prime}(x)=c_{2},
$$


onde $c_{1}, c_{2} \in \mathbb{R}$. Segue, então, que

$$
\left\{\begin{array}{l}
f(z)=c_{1} z+c_{3} \\
g(z)=c_{2} z+c_{4} \\
n(x)=-c_{1} x+c_{5} \\
w(x)=-c_{2} x+c_{6}
\end{array}\right.
$$

onde $c_{3}, c_{4}, c_{5}, c_{6} \in \mathbb{R}$. Usando A.21 obtemos que

$$
a_{y}(y, z)=c_{1} z+c_{3}=h^{\prime}(y) z+k^{\prime}(y)
$$

$\log$,

$$
h^{\prime}(y)=c_{1} \quad \text { e } \quad k^{\prime}(y)=c_{3} .
$$

Portanto, existem $c_{7}, c_{8} \in \mathbb{R}$ tais que:

$$
\left\{\begin{array}{l}
h(y)=c_{1} y+c_{7}, \\
k(y)=c_{3} y+c_{8} .
\end{array}\right.
$$

Consequentemente, de A.20d e A.21, segue que $c_{2}=c_{7}$ e $c_{4}=c_{8}$. Agora de A.20d temos

$$
c_{1}=0 \quad \text { e } \quad l(x)=-c_{5} z+c_{9}, c_{9} \in \mathbb{R}
$$

e, portanto,

$$
\left\{\begin{array}{l}
a(x, y)=c_{3} y+c_{2} z+c_{4}, \\
b(x, z)=-c_{3} x-c_{5} z+c_{9}, \\
c(x, y)=c_{5} y-c_{2} x+c_{6} .
\end{array}\right.
$$

Observação A.2.11. A partir da equação A.17 obtemos que uma base de campos de Killing em $\mathbb{R}^{3}$ é dada por:

$$
\left\{\partial_{x}, \partial_{y}, \partial_{z}, x \partial_{y}-y \partial_{x}, y \partial_{z}-z \partial_{y}, z \partial_{x}-x \partial_{z}\right\}
$$

onde $x, y, z$ denotam as coordenadas globais de $\mathbb{R}^{3}$. 
[1] A.T. Ali, R. López. Slant helices in Minkowski space $\mathbb{E}_{1}^{3}$. Korean Math. Soc. 48 (2011), 159-167.

[2] M. Barros. General helices and a Theorem of Lancret. Proc. Amer. Math. Soc. 125 (1997), 1503-1509.

[3] L. Bérard-Bergery, J.P. Bourguignon. Laplacians and Riemannian submersions with totally geodesic fibres. Illinois J. Math. 26 (1982), 181-200.

[4] M. Berger. Les variétés riemanniennes homogènes normales simplement connexes à courbure strictement positive. Ann. Scuola Norm. Sup. Pisa. 15 (1961), 179-246.

[5] L. Bianchi. Gruppi continui e finiti. Ed. Zanichelli, Bologna (1928).

[6] W. Boothby. An introduction to differentiable manifolds and Riemannian geometry. Academic Press (1986).

[7] É. Cartan. Leçons sur la géométrie des espaces de Riemann. Gauthier Villars, Paris (1946).

[8] P. Cermelli, A.J. Di Sacala. Constant-angle surfaces in liquid crystals. Phil. Mag. 87 (2007), 1871-1888.

[9] J.T. Cho, J. Inoguchi, J.E. Lee. Slant curves in Sasakian space form. Aust. Math. Soc. 74 (2006), 359-367. 
[10] M. Dajczer. Submanifolds and isometric immersions. Publish or Perish, Houston (1990).

[11] B. Daniel. Isometric immersions into 3-dimensional homogeneous manifolds. Comment. Math. Helv. 82 (2007), 87-131.

[12] F. Dillen, J. Fastenakels, J. Van der Veken, L. Vrancken. Constant-angle surfaces in $\mathbb{S}^{2} \times \mathbb{R}$. Monatsh. Math. 152 (2007), 89-96.

[13] F. Dillen, M.I. Munteanu. Constant-angle surfaces in $\mathbb{H}^{2} \times \mathbb{R}$. Bull. Braz. Math. Soc. (N.S.) 40 (2009), 85-97.

[14] A.J. Di Scala, G. Ruiz-Hernández. Helix submanifolds of Euclidean spaces. Monatsh. Math. 157 (2009), 205-215.

[15] M.P. do Carmo. Riemannian Geometry. Birkhäuser, Basel (1993).

[16] J. Fastenakels, M.I. Munteanu, J. Van der Veken. Constant angle surfaces in the Heisenberg group. Acta Math. Sin. (Engl. Ser.) 27 (2011), 747-756.

[17] C.B. Figueroa. Geometria das subvariedades do grupo de Heisenberg. Ph.D. thesis, Unicamp (1996).

[18] G. Fubini. Sugli spazi che ammettono un gruppo continuo di movimenti. Ann. di Mat., Tomo 8, serie III (1903).

[19] D. Gromoll, W. Klingenberg, W. Meyer. Riemannsche Geometrie im Grossen. Lecture Notes in Mathematics n. 55, Springer, New York (1968).

[20] J. Inoguchi, S. Lee. Null curves in Minkowski 3-space. Int. Electron. J. Geom. 1 (2008), 40-83.

[21] J. Jost. Riemannian Geometry and Geometric Analysis. Springer, fourth edition (2006).

[22] S. Kobayashi. Transformations Groups in Differential Geometry. Springer Verlag (1972)

[23] R. López, M.I. Munteanu. On the geometry of constant angle surfaces in $\mathrm{Sol}_{3}$. Kyushu J. Math. 65 (2011), 237-249. 
[24] S. Montaldo, I.I. Onnis. Helix Surfaces in the Berger Sphere. Israel J. Math. 201 (2014), no. 2, 949-966.

[25] S. Montaldo, I.I. Onnis, A.P. Passamani. Constant angle surfaces in the special linear group. Ann. Mat. Pura Appl., DOI: 10.1007/s10231-014-0452-0.

[26] M.I. Munteanu, A.I. Nistor. A new approach on constant angle surfaces in $\mathbb{E}^{3}$. Turkish J. Math. 33 (2009), 169-178.

[27] M.I. Munteanu, A.I. Nistor. Surfaces in $\mathbb{E}^{3}$ making constant angle with Killing vector fields. Internat. J. Math. 23, (2012), 16 pp.

[28] P. Petersen. Riemannian Geometry. Springer-Verlag, New York, (1998).

[29] G. Pirino. Classificazione secondo Bianchi delle metriche Riemanniane tridimensionali in base alla dimensione del gruppo delle isometrie. Tesi di Laurea. Università degli Studi di Cagliari (1989).

[30] P. Piu. Sur certains types de distributions non-intégrables totalement géodésiques. Thèse de Doctorat, Université de Haute-Alsace, Mulhouse (1988).

[31] G. Reeb. Sur certaines propriétés topologiques des trajectoires des systémes dynamiques. Acad. Roy. Belgique. Cl. Sci. Mém. 27 (1952).

[32] D.J. Struik. Lecture on Classical Differential Geometry. Diver, New-York (1988).

[33] H. Urakawa. On the least positive eigenvalue of the Laplacian for compact group manifolds. J. Math. Soc. Japan 31 (1979), 209-226.

[34] G. Vranceanu. Leçons de géométrie differentielle. Ed. Acad. Rep. Pop. Roum., vol. I, Bucarest (1957).

[35] A. Weinstein. Distance spheres in complex projective spaces. Proc. Amer. Mat. Soc., 39 (1973), 649-650. 